\title{
Nomenclature for congenital and paediatric cardiac disease: the International Paediatric and Congenital Cardiac Code (IPCCC) and the Eleventh Iteration of the International Classification of Diseases (ICD-11)*
}

Rodney C. G. Franklin, ${ }^{1}$ Marie J. Béland, ${ }^{2}$ Steven D. Colan, ${ }^{3}$ Henry L. Walters III, ${ }^{4}$ Vera D. Aiello, ${ }^{5}$ Robert H. Anderson, ${ }^{6}$ Frédérique Bailliard, ${ }^{7}$ Jeffrey R. Boris, ${ }^{8}$ Meryl S. Cohen, ${ }^{8}$ J. William Gaynor, ${ }^{8}$ Kristine J. Guleserian, ${ }^{9}$ Lucile Houyel, ${ }^{10}$ Marshall L. Jacobs, ${ }^{11,12}$ Amy L. Juraszek, ${ }^{13}$ Otto N. Krogmann, ${ }^{14}$ Hiromi Kurosawa, ${ }^{15}$ Leo Lopez, ${ }^{9}$ Bohdan J. Maruszewski, ${ }^{16}$ James D. St. Louis, ${ }^{17}$ Stephen P. Seslar, ${ }^{18}$ Shubhika Srivastava, ${ }^{19}$ Giovanni Stellin, ${ }^{20}$ Christo I. Tchervenkov, ${ }^{21}$ Paul M. Weinberg, ${ }^{8}$ Jeffrey P. Jacobs ${ }^{11,12}$ On behalf of the International Society for Nomenclature of Paediatric and Congenital Heart Disease

${ }^{1}$ Paediatric Cardiology Department, Royal Brompton \& Harefield NHS Trust, London, United Kingdom; ${ }^{2}$ Division of Paediatric Cardiology, The Montreal Children's Hospital of the McGill University Health Centre, Montréal, Québec, Canada; ${ }^{3}$ Boston Children's Hospital, Boston, Massachusetts, United States of America; ${ }^{4}$ Children's Hospital of Michigan, Wayne State University School of Medicine, Detroit, Michigan, United States of America; ${ }^{5}$ Heart Institute (InCor), University of São Paulo School of Medicine, São Paulo, Brazil; ${ }^{6}$ Institute of Genetic Medicine, Newcastle University, Newcastle-upon-Tyne, United Kingdom; ${ }^{7}$ Bailliard Henry Pediatric Cardiology, Raleigh, North Carolina, United States of America; ${ }^{8}$ Children's Hospital of Philadelphia, Perelman School of Medicine, University of Pennsylvania, Philadelphia, Pennsylvania, United States of America; ${ }^{9}$ Nicklaus Children's Hospital, Miami, Florida, United States of America; ${ }^{10}$ Department of Congenital Cardiac Surgery, Marie-Lannelongue Hospital, Le Plessis-Robinson, France; ${ }^{11}$ Department of Surgery, Division of Cardiac Surgery, Jobns Hopkins Medical Institutions, Jobns Hopkins University, Baltimore, Maryland, United States of America; ${ }^{2}$ Division of Cardiovascular Surgery, Johns Hopkins All Children's Heart Institute, Johns Hopkins All Children's Hospital, Saint Petersburg, Tampa, and Orlando, Florida, United States of America: ${ }^{13}$ College of Medicine Jacksonville, University of Florida, Jacksonville, Florida, United States of America; ${ }^{14}$ Paediatric Cardiology - Congenital Heart Disease, Heart Center Duisburg, Duisburg, Germany; ${ }^{15}$ Cardiovascular Surgery, Tokyo Women's Medical University, Tokyo, Japan; ${ }^{16}$ Department for Paediatric and Congenital Heart Surgery, Children's Memorial Health Institute, Warsaw, Poland; ${ }^{17}$ Department of Surgery, University of Missouri-Kansas City School of Medicine, Kansas City, Missouri, United States of America; ${ }^{18}$ Department of Pediatrics, Division of Pediatric Cardiology, Seattle Children's Hospital, University of Washington, Seattle, Washington, United States of America; ${ }^{19}$ Icahn School of Medicine, Children's Heart Center, Mount Sinai Hospital, New York, New York, United States of America; ${ }^{20}$ Department of Cardiothoracic and Vascular Sciences, Pediatric and Congenital Cardiac Surgical Unit, University of Padova, Padova, Italy; ${ }^{21}$ Division of Cardiovascular Surgery, The Montreal Children's Hospital of the McGill University Health Centre, Montréal, Québec, Canada

Abstract An internationally approved and globally used classification scheme for the diagnosis of CHD has long been sought. The International Paediatric and Congenital Cardiac Code (IPCCC), which was produced and has been maintained by the International Society for Nomenclature of Paediatric and Congenital Heart Disease (the International Nomenclature Society), is used widely, but has spawned many "short list" versions that differ in content depending on the user. Thus, efforts to have a uniform identification of patients with CHD using a single up-to-date and coordinated nomenclature system continue to be thwarted, even if a common nomenclature has been used as a

*Presented at The Fifth International Summit on Nomenclature for Pediatric and Congenital Heart Disease at the 2017 Seventh World Congress of Pediatric Cardiology \& Cardiac Surgery (WCPCCS 2017), Barcelona, Spain, July 16-21, 2017. Presented Sunday, July 16, 2017.

Correspondence to: Dr R. C. G. Franklin, Paediatric Cardiologist, Royal Brompton \& Harefield NHS Trust, London SW3 6NP, United Kingdom. Tel: +44 2073518613 ; Fax: +44 1895 826589; E-mail: r.franklin@rbht.nhs.uk 
basis for composing various "short lists". In an attempt to solve this problem, the International Nomenclature Society has linked its efforts with those of the World Health Organization to obtain a globally accepted nomenclature tree for CHD within the 11th iteration of the International Classification of Diseases (ICD-11). The International Nomenclature Society has submitted a hierarchical nomenclature tree for CHD to the World Health Organization that is expected to serve increasingly as the "short list" for all communities interested in coding for congenital cardiology. This article reviews the history of the International Classification of Diseases and of the IPCCC, and outlines the process used in developing the ICD-11 congenital cardiac disease diagnostic list and the definitions for each term on the list. An overview of the content of the congenital heart anomaly section of the Foundation Component of ICD-11, published herein in its entirety, is also included. Future plans for the International Nomenclature Society include linking again with the World Health Organization to tackle procedural nomenclature as it relates to cardiac malformations. By doing so, the Society will continue its role in standardising nomenclature for $\mathrm{CHD}$ across the globe, thereby promoting research and better outcomes for fetuses, children, and adults with congenital heart anomalies.

Keywords: Congenital heart disease; classification; ICD-11; WHO; IPCCC

Received: 21 November 2017; Accepted: 5 December 2017

C INICIANS WORKING IN THE FIELD OF CONGENITAL and paediatric cardiology have long felt the need for a common diagnostic and therapeutic nomenclature and coding system with which to classify patients of all ages with congenital cardiac disease. In the early 2000s, this desire was heightened by high-profile enquiries examining the outcome of paediatric cardiac surgery at the congenital cardiology centres in Bristol, United Kingdom ${ }^{1,2}$; Sydney, Australia $^{3}$; Winnipeg, Canada ${ }^{4}$; Denver, Colorado ${ }^{5,6}$; and Lexington, Kentucky. ${ }^{7}$

The incorporation of effective clinical governance and best practice into our speciality requires the harvesting of accurate and validated data on the diagnosis, treatment, and outcome of these patients from prenatal life through to adulthood. Such data gathering facilitates the comparison of outcomes following interventions between individual centres, while taking into account the mix of cases involved, any accompanying risk factors and comorbidities, as well as postprocedural complications. Benchmarking against those units who perform best allows analysis of relevant and genuine factors underlying differing outcomes, and instigation of improvements, in terms of both mortality and morbidity. For this objective to be achieved, it is essential to have a comprehensive and standardised system of coding and classification, using mutually exclusive and unambiguous terms. The system must be easy to use, preferably in multiple languages, be digitally compatible with different software database systems, and also fulfil the needs and expectations of widely different cultures of practice.

A cohesive and comprehensive system of nomenclature, which is suitable for setting a global standard for multicentric analysis of outcomes and stratification of risk, has been published, namely the freely available International Paediatric and Congenital Cardiac Code (IPCCC), ${ }^{8}$ as developed and copyrighted by the
International Society for Nomenclature of Paediatric and Congenital Heart Disease (International Nomenclature Society). The IPCCC is available for download without cost from the Society's website at http://www. IPCCC.net. The IPCCC has been used extensively as the coding system underlying international and national comparisons of outcomes between individual centres undertaking congenital heart surgery and transcatheter interventions. $^{9-12}$

Unfortunately, while coding systems such as the IPCCC have been used by specialist clinicians for quality assurance, outcome analysis, and research, health administrators often follow their own coding schemes, most commonly the International Classification of Diseases (ICD) of the World Health Organization. In so doing, efforts are thwarted to have a uniform identification of patients with CHD using a single up-to-date and coordinated nomenclature system.

An ideal classification scheme for CHD would be one that could serve the purposes of both administrators and clinicians. In the last decade, the development of such a scheme was made possible when it was announced that a new 11th iteration of the International Classification of Diseases (ICD-11) was planned, and that input from clinicians and researchers was sought. After discussion with senior representatives of the ICD-11 revision team in 2007, the International Nomenclature Society was given the mandate to provide a list of CHD to be incorporated into ICD-11, and in 2010 the Society set about achieving this task. The final product of 7 years of deliberation is published in this issue of Cardiology in the Young.

This article reviews the history of the International Classification of Diseases, and of the IPCCC. It discusses the content of the congenital cardiology section of ICD-11, a classification tree that will permit the widespread use of a single diagnostic list for CHD both for administrative and clinical purposes. 


\section{The International Classification of Diseases ${ }^{13}$}

Although the gathering of population-based information, or censuses, originated in Babylonian times (3800 BC), it was not until the early 17 th century that the focus shifted away from taxation and military conscription to causes of death, with the weekly publication from 1603 through the 1830s of the Bills of Mortality in London. ${ }^{14}$ In 1662, these statistics were first systematically analysed by John Graunt (1620-1674), when he estimated, for instance, the mortality of children before the age of 6 years to be $36 \%$, an estimate later proven to be quite accurate.

In 1837, the General Register Office of England and Wales was established, with William Farr (1807-1883) as its first medical statistician. Farr revolutionised the principles of statistical classification and nomenclature, emphasising the need for a common international lexicon to allow the epidemiological study of diseases and thereby their causes, while incorporating medical advances. His initial report, published in 1839, resonates through to the late 20th century:

"The advantages of a uniform statistical nomenclature, however imperfect, are so obvious, that it is surprising that no attention has been paid to its enforcement in Bills of Mortality. Each disease has, in many instances, been denoted by three or four terms, and each term has been applied to as many different diseases: vague inconvenient names have been employed, or complications registered instead of primary diseases. The nomenclature is of as much importance in this department of inquiry, as weights and measures in the physical sciences, and should be settled without delay."

The eminence of Farr in the field was recognised at the first International Statistical Congress, held in Brussels in 1853, when he proposed a classification of causes of mortality based on five groups, which formed the basis of the 139 categories accepted by the Congress in 1855:

- epidemic diseases

- constitutional, or general, diseases

- local diseases arranged according to anatomical site

- developmental diseases

- diseases that are the direct result of violence.

These held for the subsequent four revisions through to 1886 , and the later International Classification of Diseases, particularly the principle of classifying causes of death by anatomical site, in distinction to generalised processes of disease. As there was limited take-up of this classification, in 1891 the International Statistical Institute (the former Congress) commissioned a committee chaired by Jacques Bertillon (1851-1922), Chief of Statistical Services of the City of Paris, to create what became the Bertillon [International] Classification of Causes of Death, based on the principles propounded by Farr, with associated sequential numeric codes. ${ }^{16}$ Over the following decades, this classification scheme was adopted by many countries in the Americas and Europe, with conferences for revision occurring roughly decennially to take note of medical advances (Fig 1).

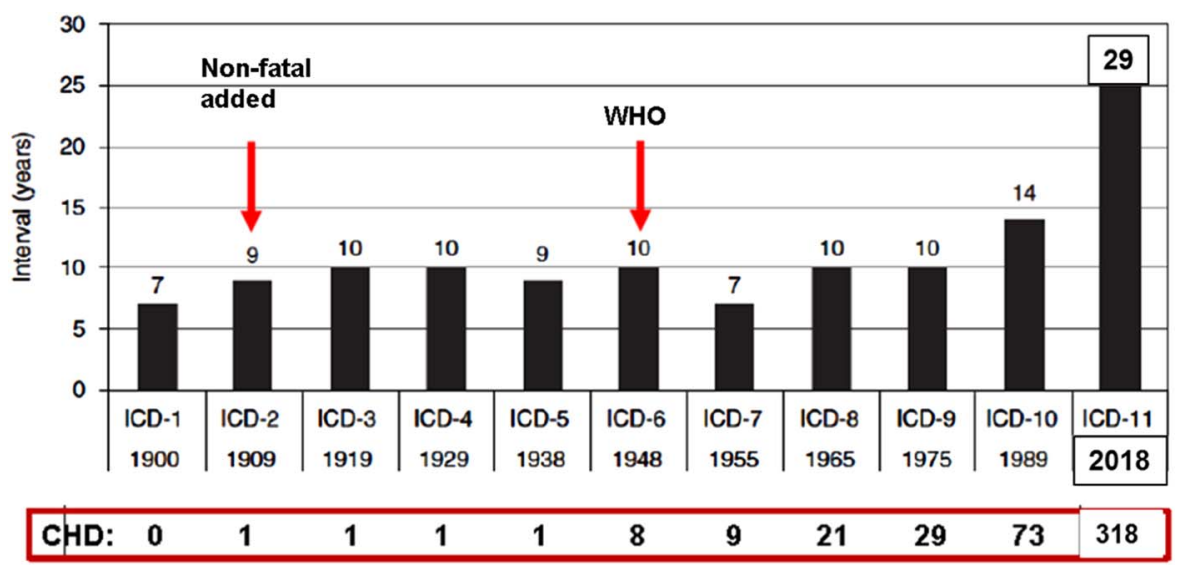

Figure 1.

This bar chart documents the time interval between each Revision of the International Classification of Diseases (ICD), 1900-2018. Bertillon presented his (International) Classification of Causes of Death at the meeting of the International Statistical Institute in Chicago in 1893, where it was adopted by several cities and countries. In 1898, the American Public Health Association recommended its adoption in North America and that the classification be revised every 10 years. The First International Conference to revise the Bertillon Classification of Causes of Death was held in Paris in 1900. In 1909 non-fatal diseases were added (morbidity) and in 1948 the World Health Organization (WHO) took over its promotion and management with the sixth revision of the International Classification of Diseases, Injuries and Causes of Death. The horizontal lower bar indicates the number of CHD terms listed in each ICD version. 
Farr was also instrumental in promoting the need for a similar system to account for non-fatal disease, submitting a list of these to the second International Statistical Congress in 1856. He stated the need to

"extend the same system of nomenclature to diseases which, though not fatal, cause disability in the population, and now figure in the tables of the diseases of armies, navies, hospitals, prisons, lunatic asylums, public institutions of every kind, and sickness societies, as well as in the census of countries like Ireland, where the diseases of all the people are enumerated." ${ }^{\prime 17}$

At the fourth International Statistical Congress held in 1860, Florence Nightingale urged its adoption for the tabulation of hospital morbidity in her paper Proposals for a uniform plan of hospital statistics. ${ }^{18}$ In 1900 and 1909, at the first two Conferences to revise the Bertillon International Classification of Causes of Death, a parallel classification of diseases for use in statistics of sickness was adopted, but with limited scope. The Health Organization of the League of Nations, and the International Statistical Institute, recommended at the fourth and fifth revision conferences of the International List of Causes of Death as the International Classification of Diseases was then known, held in 1928 and 1938, respectively, that individual countries develop and promote their own systems of classification for morbidity statistics, using the Causes of Death structure as a template. It was not until the sixth revision conference, held in Paris in 1948, when the recently constituted World Health Organization took over its promotion and management, that a single comprehensive list was endorsed by the First World Health Assembly as the International Classification of Diseases, Injuries and Causes of Death, the sequential four-digit code being augmented by lists of causes of accidents and injuries (E-codes). The ninth International Classification of Diseases revision (ICD-9), in 1975, saw further expansion to nearly 18,000 codes, as well as structural change, with a new five-digit sequential numeric designation for each entity, the optional fifth digit facilitating greater detail where needed by subspecialities. The dagger and asterisk notation system was introduced to allow the specific linkage of anatomical sites of disease to more generalised diseases. The first International Classification of Procedures in Medicine was also published, in response to international pressure for a lead in this important area.

By the time of the tenth revision (ICD-10), in 1993, the decennial revision schedule was abandoned (Fig 1) because of the enormous amount of work and international consultation involved with each revision, and a new chapter-based alphanumeric coding structure was introduced, retaining the overall four-digit structure. ICD-10, whose full name is International Statistical Classification of Diseases and Related Health Problems, consists of over 14,400 basic codes, and is currently used in 115 countries for reporting mortality. It has been translated into 43 languages, with the code ensuring that the data derived are language independent. Adoption of ICD-10 has been very variable, with the United States of America only abandoning ICD-9 in 2015. This is largely because of the expense of adapting data processing systems with each ICD revision, and despite the fact that World Health Organization member states are expected to use the most current version of ICD to report death and illness, as agreed by an international treaty in 1967. Many countries have created their own versions of ICD-9 and ICD-10 to produce Clinical Modifications (ICD-9-CM, ICD-10-CM), so as to expand the lexicons sufficiently for their own bespoke clinical use and administrative purposes, such as billing. In the United States Clinical Modification, for example, there are over 68,000 ICD-CM codes with an up to seven-digit coding structure. The International Classification of Diseases is one of the World Health Organization Family of International Classifications designed to support national and international health information systems, statistics, and evidence. ${ }^{19}$ The other two key World Health Organization classification systems are the International Classification of Functioning, Disability and Health, and the International Classification of Health Interventions, which is under development.

\section{The IPCCC}

The development of classification schemes specific to the malformed heart has a shorter history, which began with Maude Abbott's pioneering work in Montreal in the early 1900s. Her 1936 Atlas of Congenital Cardiac Disease was the first formal attempt to classify CHD. ${ }^{20}$ Following this initial effort, centres across the world developed or adopted different schemes to produce more hospital-specific databases. By the late 20th century, a multitude of centre-derived congenital heart classification systems for internal, national, and even international comparisons were developed, but these were dissimilar enough to preclude the large-scale studies needed to benchmark the outcomes of the heterogeneous population of patients with congenitally malformed hearts. Aided by advances in information technology to facilitate the entry of data and exchange of information, two independent international endeavours began in the 1990s and resulted in the 
near simultaneous publication in 2000 of two separate congenital cardiology nomenclature lexicons ${ }^{21}$ :

1. The European Paediatric Cardiac Code of the Association for European Paediatric and Congenital Cardiology 22,23

2. The nomenclature system of the International Congenital Heart Surgery Nomenclature and Database Project of the Society of Thoracic Surgeons in North America, and the European Congenital Heart Defects Database of the European Congenital Heart Surgeons Foundation - renamed the European Congenital Heart Surgeons Association in $2003 .{ }^{24,25}$

Both systems of nomenclature included a comprehensive Long List, with thousands of terms, and a so-called Short List designed to be used as part of a minimum data set for audit and research purposes. Both Long Lists mapped fully to their respective Short Lists. The nearly simultaneous publication of these two complementary systems of nomenclature led to the problematic situation of having two lexicons that were to be widely adopted, with the potential risks of invalidating multicentric projects owing to confusion between the two systems, and duplicate or inaccurate entries within institutions.

Following a meeting between the involved society representatives in October, 2000 in Frankfurt, Germany, the International Nomenclature Committee for Paediatric and Congenital Heart Disease was established to include representatives of the three societies, as well as representatives from the remaining continents of the world - Africa, Australia, Asia, and South America. It was tasked to work in partnership and produce a reconciliatory bidirectional map between the two systems. In 2005, the International Nomenclature Committee became the International Nomenclature Society and was legally incorporated as a not-for-profit corporation in Canada (Fig 2). Fortuitously, the International Congenital Heart Surgery Nomenclature and Database Project did not feature a numerical code, and the six-digit numerical code derived from the European Paediatric Cardiac Code was used as the backbone for mapping the two systems.

The feasibility of this project was established by the creation of a rule-based bidirectional crossmap between the two Short Lists by the Nomenclature Working Group of the International Nomenclature Society. ${ }^{26,27}$ Over the next 8 years, the Nomenclature Working Group met 10 times, over a combined period of 47 days, to achieve the main goal of mapping the two comprehensive lists to each other to create the two dominant versions of IPCCC. ${ }^{28}$

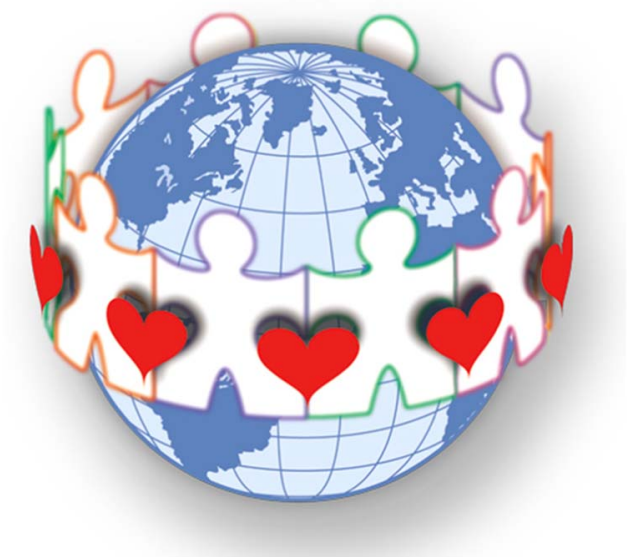

Figure 2.

The logo of the International Society for Nomenclature of Paediatric and Congenital Heart Disease.

- The IPCCC derived from the European Paediatric Cardiac Code of the Association for European Paediatric and Congenital Cardiology;

- The IPCCC derived from the International Congenital Heart Surgery Nomenclature and Database Project of the Society of Thoracic Surgeons, the European Congenital Heart Surgeons Association, and the European Association for Cardio-Thoracic Surgery.

For all terms within the two versions of the IPCCC, a unique six-digit code corresponds to a single entity, whether it be a morphological phenotype, procedure, symptom, or genetic syndrome, with the mapped terms in each of the two versions being synonymous to each other. By 2013, there were 12,168 items in the IPCCC version derived from the European Paediatric Cardiac Code, and 17,176 in the IPCCC version derived from the International Congenital Heart Surgery and Nomenclature Database Project, with additional hundreds of qualifiers, some specific (such as anatomical sites), and some generic (such as gradings of severity).

It is primarily the Short Lists, rather than the Long Lists, of the two crossmapped versions of the IPCCC that have been used for analyses of multi-institutional and international outcomes following operations and procedures for patients with congenitally malformed hearts, with close to a million patients in registries worldwide. Both versions of the IPCCC Short Lists have been used to develop empirical systems for the stratification of risk following surgical procedures, based on the operation type, comorbidities, and diagnoses, for quality assurance and quality improvement purposes. ${ }^{29-31}$ Both risk adjustment systems depend upon the IPCCC for all variables, to ensure a common lexicon of terms between 
institutions submitting data, and both perform better than the systems based on the subjective assessment of risk.

Although the Long Lists of each version have been crossmapped, the two Short Lists emanating from their respective Long-List versions are not the same in terms of structure or content. One Short List has a distinct surgical orientation, with the other serving more cardiological needs. The International Nomenclature Society recognised this disparity, and believed that the creation of a congenital cardiology subset within ICD-11 would help resolve the situation by presenting a single comprehensive and hierarchical list of diagnostic terms that could serve all communities dealing with CHD.

\section{The ICD-11}

Apart from the treaty-bound necessity of the World Health Organization "to establish and revise as necessary international nomenclatures of diseases, of causes of death and of public health practices", ${ }^{32}$ ICD-10 is no longer fit for purpose to address the needs of the digital healthcare systems in the 21 st century with the move to the electronic health record and a paperless environment. This requires a standardised system for gathering and retaining accurate and detailed clinical information, free from duplication of data entry, enabling comparative studies across units and nations of clinical and cost-related outcomes for risk-adjusted benchmarking to best practice. The prerequisite for this accomplishment is an underlying, all encompassing, common nomenclature and system of coding for healthcare, with clinician-led and validated entry of data. The ICD-11 mission, therefore, is "To produce an international disease classification that is ready for electronic health records that will serve as a standard for scientific comparability and communication". ${ }^{33}$ Its purpose is to allow the systematic recording, analysis, interpretation, and comparison of mortality and morbidity data collected in different countries or areas and at different times. The ICD-11 project began in earnest in 2007 and is planned to achieve its first stage of implementation in May, 2018, after endorsement at the 71st World Health Assembly of the ICD-11 version for Mortality and Morbidity Statistics, known as ICD-11-MMS. Uniquely for the International Classification of Diseases, it incorporates textual definitions, and there are logical linkages to other standard terminologies, particularly the Systematised Nomenclature of Medicine Clinical Terms (SNOMED-CT). ${ }^{32}$ In addition, the system is to be explicitly stratified to cater to different users, such as primary care, traditional medicine, and public health, producing so-called linearisations or "Tabular Lists".
For the first time, the revision process has moved away from reliance on large meetings of national delegations of health statisticians with manual archiving - curation, limited field testing, working solely in the English language, wherein those who voiced their opinion strongest would dominate the content of the paper-based output - "decibel" diplomacy. In contrast, the ICD-11 revision process is dependent upon international expert clinicians, with digital curation, the incorporation of wide peer review, and extensive field testing. Although working in English, multiple translations are integral to the process. The work was divided into contentspecific Topic Advisory Groups, with related Working Groups led by Managing Editors and chaired by specialist clinicians with an intentionally wide geographic spread (Fig 3). From 2009 through to 2016, the Managing Editor coordinated a series of meetings, some face-to-face but mostly teleconferences, to populate and update the classification of specialist areas, beginning with the hierarchical structure and terms within ICD-10, and initially producing an evolving alpha draft. In 2012, a beta draft was published online (https://icd.who.int/dev11/f/en), coinciding with the authoring process moving to a webbased platform for its entire content. This is known as the ICD Collaborative Authoring Tool (iCAT), whereby the ICD-11 content and structure is enhanced and edited by the designated Managing Editors. ${ }^{33}$ The tool also allows online global peer review and submission of comments by both the authors and worldwide interested parties in the current field-testing stage.

From the start, clinicians involved in the Topic Advisory Groups have been encouraged to enlist the advice of specialist Societies to aid the process, and to ensure that the content was both up-to-date and had Societal endorsement. This has resulted in a huge increase in the number of individual terms within ICD-11, with secondary expanse of the hierarchical structure when compared with ICD-10. An example is congenital cardiology, with an increase from 73 CHD diagnostic terms in ICD-10 to 318 codes submitted by the International Nomenclature Society for incorporation into ICD-11. Populating ICD-11 by the content-specific Topic Advisory Groups was not always without controversy, with at times, for example, heated and prolonged discussions between the Rare Diseases Topic Advisory Group and several Internal Medicine Topic Advisory Workings Groups, including the Cardiovascular Working Group, over the hierarchy to be included or excluded.

ICD-11 uses an ontological content model derived from computer science. Ontology in this context can be defined as the explicit, operational description of the concepts within a domain - in other words, its 


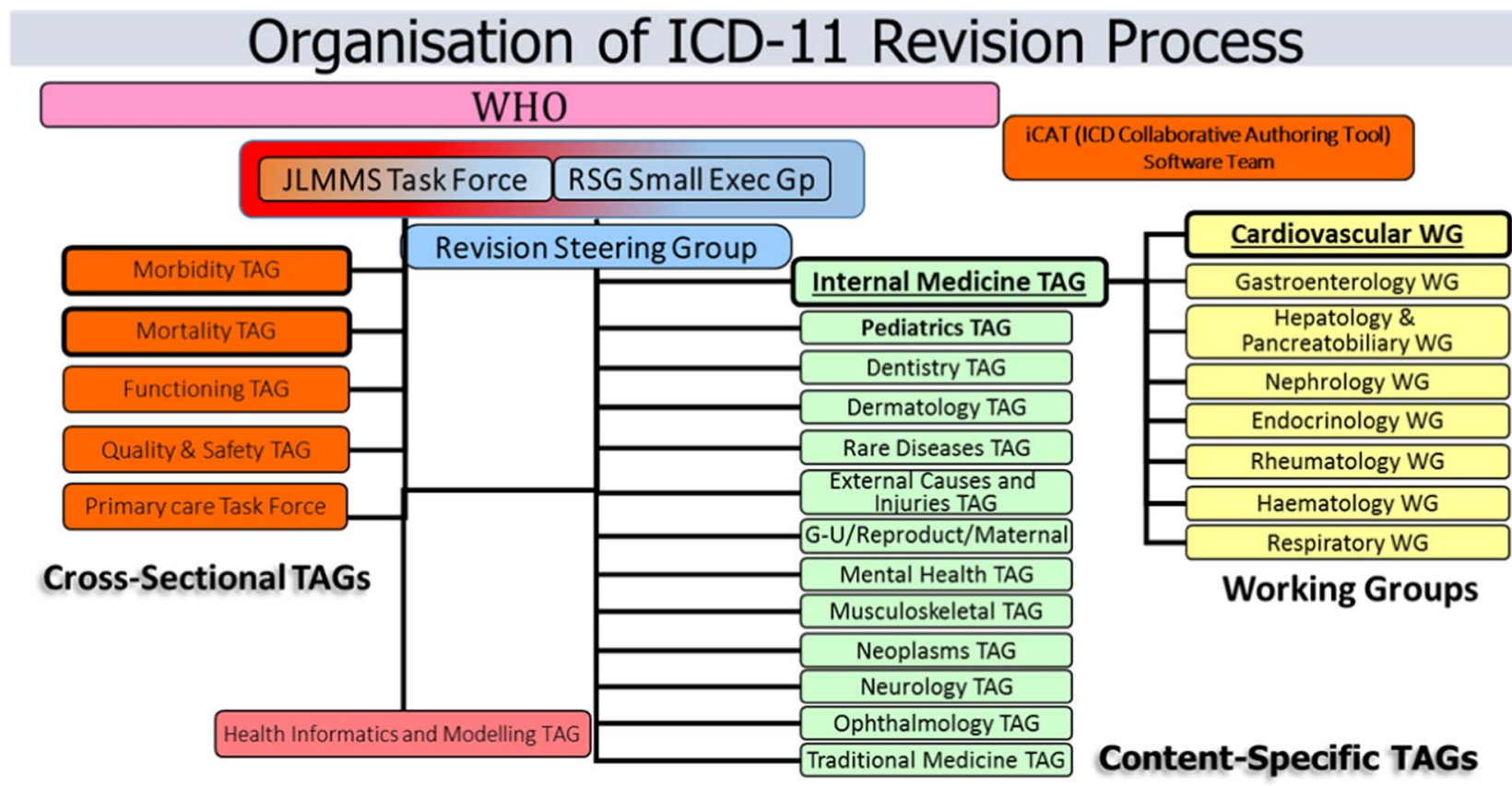

Figure 3.

Diagram illustrating the workflow for the collaborative authoring ICD-11 revision process, led by the Revision Steering Group and its Small Executive Group. Branches of ICD classification are assigned to at least one Content-Specific Topic Advisory Group (TAG), each led by a Managing Editor and two clinical chairs. Congenital cardiology falls under the remit of the Cardiovascular Working Group of the Internal Medicine TAG with collaborative input and ratification from the Pediatric TAG. The members of each working group and TAG have the task of entering the content of the diseases in their domain of expertise, such as textual definition, synonyms, and so on. Cross-Sectional TAGs are involved with specific linearisations for their area, using terms from the Content-Specific TAGs as appropriate, the dominant one being the Joint Linearization for Morbidity and Mortality Statistics (JLMMS). Data entry by Managing Editors uses the ICD Collaborative Authoring Tool (iCAT) web platform, which can also be used for commentary and feedback during field testing.

qualities, properties, and attributes. In ICD-11, the evidence-based attributes of each individual disease are delineated, with the current emphasis on textual definitions, synonyms, inclusions, and exclusions. There is the ambition to extend the content to include diagnostic criteria, genetics, causal mechanisms, functional impact, and so on. This ontological content model will result in a common vocabulary with robust data standards, enabling a shared understanding and exchange of information (data) between individuals and institutions, permitting interoperability and like-for-like comparisons across all fields of medicine.

An important additional feature in ICD-11 is the ability to add a large-range additional clinically useful detail to a primary diagnosis or "stem code", using "extension codes", to produce a "cluster code", also called post-coordination. Extension codes are housed in a specific ICD-11 Chapter (Chapter X) and include details of severity, size, temporality, aetiology, for example pathogen causing infection, anatomical detail, type of device related to a postprocedural complication, and so on. These equate to qualifier codes in the IPCCC. Other extension codes cover more administrative and broader health interactions, such as reason for health encounter/admission, for example illness, screening, diagnosis confirmed/ ruled out, and patient related such as family history of a condition. Of course not all extension codes can be used with every stem code. It is planned that each stem code will have a list of valid extension codes visible for use when such detail is available and required for patient management or research purposes.

\section{ICD-11 and the International Nomenclature Society}

Up until the ICD-11 iteration, congenital cardiac diseases remained very poorly represented. Outdated terminology, inconsistent logic, and little appreciation of the inherent complexity of lesions have meant that the ICD system of classification has never been robust enough for the purposes of relevant clinical governance. In the ninth and tenth revisions, there are a total of only 29 and 73 individual codes, respectively, for congenital cardiovascular lesions (Fig 1). Evidence suggests that the validity of coding of lesions seen in the congenitally malformed heart via the ninth revision of the International Classification of Diseases (ICD-9), as currently used in 
administrative databases in the United States, is poor. ${ }^{34-38}$ For example, in a series of 373 infants with congenital cardiac defects at Children's Hospital of Wisconsin, investigators reported that only $52 \%$ of the cardiac diagnoses in the medical records had a corresponding code from the International Classification of Diseases in the hospital discharge database. $^{34}$ A subsequent study of infants and fetuses with cardiac defects, born in Atlanta from 1988 to 2003, compared administrative coding using ICD-9 and the clinical nomenclature used in the Society of Thoracic Surgeons Congenital Heart Surgery Database. $^{36}$ This study concluded that analyses based on the 29 codes available in ICD-9 are likely to "have substantial misclassification" of congenital cardiac disease. This prompted the organisation of a linkage study using patient data (2004-2010) from the Society of Thoracic Surgeons Congenital Heart Surgery (STS-CHS) Database, a clinical registry, and the Pediatric Health Information Systems administrative database in order to evaluate differential coding/ classification of operations between data sets and subsequent impact on outcomes assessment. ${ }^{37}$ The cohort included 59,820 patients from 33 centres. There was a greater than $10 \%$ difference in the number of cases identified between data sources for half of the benchmark operations. The negative predictive value of the administrative, versus clinical, data was high (98.8-99.9\%); the positive predictive value was lower (56.7-88.0\%). These differences translated into significant differences in case ascertainment and outcomes assessment. These ranged from an underestimation of mortality associated with common arterial trunk repair by $25.7 \%$ in the administrative versus clinical data $(7.01 \%$ versus $9.43 \% ; \mathrm{p}=0.001)$ to an overestimation of mortality associated with ventricular septal defect repair by $31.0 \%(0.78 \%$ versus $0.60 \% ; p=0.1)$. An extension to this study demonstrated similar concerns about federal recommendations in the United States of America that suggest administrative data be used to assess hospital-level mortality rates as a measure of centre performance. ${ }^{38}$ Surgical volume was underreported, with lower mortality using administrative data compared with registry data, with rank positions differing by at least five positions for eight of the 33 centres evaluated. This produced a change in mortality tertile classification - high, middle, or low mortality - for 6 units, and a change in statistical outlier classification for four hospitals. Despite their limitations with respect to $\mathrm{CHD}$, nonetheless, both ICD-9 and ICD-10 remain in use, mostly for the purpose of "billing", returns to central governments, and crude epidemiological surveillance.

Discussions began in 2007, in Tokyo Japan, between the International Nomenclature Society and representatives of the steering group responsible for the creation of ICD-11, administered by the World Health Organization. Both parties were interested in greatly expanding the CHD section within this new iteration of the International Classification of Diseases, in order to include enough terms to be useful for clinicians and researchers who work in the field of congenital cardiology. By mutual agreement, the International Nomenclature Society took on the task of developing the list of diagnostic terms for $\mathrm{CHD}$ for ICD-11 that was to include definitions and synonyms for each diagnosis.

\section{Developing the CHD list for ICD-11}

To fulfil this new mandate, the International Nomenclature Society formed the Definitions Working Group, which was tasked with creating a comprehensive list of diagnostic terms to be used in ICD-11 to describe CHD in sufficient detail for clinical use by specialists in the field. This was to be achieved by, and from the perspective of, an international body of experts from congenital and paediatric cardiology, cardiovascular surgery, and cardiac morphology.

In ICD-11, congenital and paediatric cardiac diseases are under the auspices of the Cardiovascular Working Group of the Internal Medicine Topic Advisory Group, in liaison with the Pediatric Topic Advisory Group (Fig 3). The Definitions Working Group worked therefore in liaison with both Topic Advisory Groups to produce a large subset of terms derived from the IPCCC and presented in a hierarchical manner. The Definitions Working Group was also tasked with providing definitions and synonyms for each term. Definitions were to be used further to validate the selection of different codes so as to accurately describe and code CHD in all its forms.

\section{Methodological approach to defining the ICD-11 terms}

Early in the deliberations of the Definitions Working Group of the International Nomenclature Society in 2010, considerable discussion was devoted to achieving agreement on what constitutes a useful definition. Fundamentally, the group sought definitions that provided a concise set of findings that were essential to the diagnosis, while avoiding inclusion of variable elements of the disease. For example, although right ventricular dilation is usually associated with an atrial septal defect, it is the presence of a communication between the atrial chambers that constitutes the definition. This is also an example of defining anatomic abnormalities based on morphology, whereas physiologic abnormalities such as aortic 
regurgitation are defined based on their functional abnormalities. The definitions were then systematically formulated during nine meetings of the committee in the ensuing 7 years. The group first created a systematic hierarchical list of terms headed by "congenital anomaly of heart and great vessels" (Table 1). Although the terminological hierarchy was developed first, the definitions process at times resulted in reconsideration of this hierarchy. In addition to defining the terms themselves, the definitions committee also took responsibility for development of the related elements required by the ICD-11 system. These include acceptable synonyms, unacceptable synonyms, relevant historical terms that are useful for search purposes, and abbreviations. Some areas of the congenital cardiology sections were found to be prepopulated with terms and synonyms as found in ICD-10 or input from non-specialists in congenital cardiology. These discrepancies were rectified on an item-by-item basis, submitting the rationale for change to the Managing Editor of the Internal Medicine Topic Advisory Group. The ICD-11 system also includes a "notes" or commentary option, permitting the inclusion of explanatory material to describe the phenotype in more detail, to further explain differences between individual codes, and to point the user to an appropriate alternative term (Table 2, italicised text after formal definition). This option was only occasionally used by the Definitions Working Group, although as field testing of the ICD-11 system proceeds the committee may use this section to clarify usage.

The procedural method adopted by the Definitions Working Group was to devote 2.5 to 3 days at inperson committee meetings to achieve consensus on the individual definitions. Before each meeting, each attendee was asked to develop proposed definitions for a group of terms and these preliminary definitions then served as the basis for discussion and refinement by the committee at large. Outside information, including reference to earlier literature, imaging data, intraoperative experience, and observations from pathologic specimens, was often used. The struggle for consensus was indeed a struggle, but generally with sufficient time the committee achieved agreement. There was also no policy precluding reconsideration of definitions that had previously been agreed to. Several of the more challenging terms achieved consensus definitions that were then subsequently reconsidered. The terminology and definitions for the ventricular septal defects, for example, were reconsidered at nearly all of the inperson meetings.

One of the most important obstacles to progress in establishing common terminology is the historical differences in usage at many of the centres, and the fear that deviations from the existent standards could lead to rejection of terminology that was at odds with the "preferred" local terminology. In cases in which different names are used for the same entity, a crossmapping between the terminologies can be used to avoid conflicts, effectively treating the terms as synonyms, as the code itself is independent of differing terminologies for the same phenotype. Many of these synonyms have been documented as such in the ICD-11 system to help avoid this conflict, as shown in Table 2. When the same name has been used to refer to different entities, however, the committee found it preferable to drop the name altogether. Inevitably, there are also cases in which there is fundamental disagreement as to the specific morphologic or physiologic nature of the disease, leading different experts to conclude that specific names are wrong. Despite these differences, generally a cross-mapping of these terms is possible such that, although unresolvable divergences of opinion will persist, the ability to appropriately group these entities for epidemiological and outcome analyses can still be preserved.

After 7 years the Definitions Working Group of the International Nomenclature Society began working on ICD-11 congenital cardiology content, the Group has submitted to the World Health Organization a list of over 300 diagnostic terms, all items being based upon the IPCCC and accompanied by definitions and synonyms. This list of CHD terms, along with definitions, synonyms, and the IPCCC six-digit numerical codes, is presented in this issue of Cardiology in the Young. It is accessible on the World Health Organization website in its beta draft form https://icd.who.int/dev11/f/en\#/, under 'Diseases of the circulatory system', and then 'Developmental anomalies of the circulatory system'. They are also available on the International Nomenclature Society website www.ipccc.net, where additional lists of the IPCCC will be published with the terms contained in ICD-11 being highlighted in the long lists of both versions of the IPCCC.

\section{Navigating ICD-11 to find CHD terms, synonyms and definitions}

The full ICD-11 content is known as the Foundation Component, and represents the entire ICD universe. The diagnostic terms for CHD that were submitted by the International Nomenclature Society reside in the Foundation Component of ICD-11. The Foundation Component has been divided into 26 sections, including one on Developmental Anomalies, which includes Structural developmental anomalies of the circulatory system. The list of diagnostic terms for CHD is found under a further subsection "Structural developmental anomaly of heart and great vessels". 
It is worth noting that, unlike previous ICD versions, all Foundation Component terms are not necessarily mutually exclusive, as double parenting is permitted such that an entity may exist in more than one hierarchy. For example, heart tumours are found under the sections both for neoplasms and diseases of the circulatory system, but should retain the same numerical designation.

The list of CHD terms that has been submitted by the International Nomenclature Society to the World Health Organization is published in two tables in this issue of Cardiology in the Young. Table 1 contains the list of diagnostic terms presented in a hierarchical manner. Table 2 contains a column of the same list of terms, but includes corresponding definitions and comments for each term, synonyms, as well as the sixdigit IPCCC number that has been attributed to each term by the International Nomenclature Society. When ICD-11 is officially released, another set of ICD-specific numeric codes will be given to each term, but as yet these are not available for publication. In the discussion below, the bracketed number that follows each term is its IPCCC six-digit identification code.

As stated previously, the list of congenital heart malformations submitted by the International Nomenclature Society for incorporation into ICD-11 contains 318 terms. The hierarchy begins with the "level 0" term: Structural developmental anomaly of heart and great vessels (01.01.59). There are then 10 "level 1 " items starting with those describing position and connections, and followed, in segmental sequential order, by veins, atria, atrioventricular valves, ventricles, arterial valves, and great arteries.

1. Congenital anomaly of position or spatial relationships of thoraco-abdominal organs (03.01.13).

2. Congenital anomaly of an atrioventricular or ventriculoarterial connection (01.03.09).

3. Congenital anomaly of mediastinal vein (04.00.07).

4. Congenital anomaly of an atrium or atrial septum (05.00.02).

5. Congenital anomaly of an atrioventricular valve or atrioventricular septum (06.00.15).

6. Congenital anomaly of a ventricle or the ventricular septum (07.00.00).

7. Functionally univentricular heart (01.01.22).

8. Congenital anomaly of a ventriculo-arterial valve or adjacent regions (09.04.29).

9. Congenital anomaly of great arteries including arterial duct (09.04.28).

10. Congenital anomaly of coronary arteries (09.46.03).

Each of the 10 "level 1" terms is subdivided into finer and finer branches as needed, to a maximum of seven levels of detail and a total of 318 terms (see Table 1).
Although the CHD list is designed to enable the diagnosis of any congenital heart anomaly, 10 "normal" terms were included within the list. These are to be used when describing a complex congenital malformation, as some parts of the anomaly include normal findings. These "normal" terms are listed below, with the secondary terms denoting notable synonyms.

- Laevocardia (02.01.03)

- Usual atrial arrangement - atrial situs solitus (01.03.00)

- Right-hand pattern ventricular topology - D-loop ventricles (02.03.11)

- Aortic root posterior and rightward to pulmonary root (02.06.06)

- Spiralling course of great arteries (02.07.00)

- Concordant atrioventricular connections (01.04.01)

- Concordant ventriculo-arterial connections (01.05.00)

- Patent oval foramen - patent foramen ovale (05.03.01)

- Patent arterial duct - patent ductus arteriosus (09.27.21)

- Left aortic arch (09.28.22)

\section{What the CHD list of ICD-11 does not contain}

The section Structural developmental anomaly of heart and great vessels includes only congenital malformations. Thus, heart lesions that are not clearly congenital in nature do not appear in this section. For example, "Congenital aortic valvar stenosis" (09.15.01) appears in the structural developmental section, but "Aortic valvar stenosis" (09.15.13) does not. Terms dealing with cardiac lesions that are not necessarily congenital in nature can be found in the ICD-11 section dealing with Diseases of the Circulatory System. With few exceptions, particularly cardiac diseases that are not paediatric or congenital in nature, the cardiac terms listed in both these sections are found also in the IPCCC. Acquired cardiac lesions are also listed in Diseases of the Circulatory System within ICD-11, and those acquired cardiac lesions that pertain to CHD were developed and submitted separately for incorporation into ICD-11 by the International Nomenclature Society.

\section{Principles used in drawing up the IPCCC and the CHD section of ICD-11}

As described previously, ${ }^{26}$ members of the International Nomenclature Society followed certain principles in developing the IPCCC and they have been incorporated into the Foundation Component submission for ICD-11. 


\section{Language}

The International Nomenclature Society submitted the CHD list of terms in English. American and British spelling was used interchangeably and words with dual spellings were treated as synonyms. A French translation of the CHD list has been completed by a sub-committee of the International Nomenclature Society. The World Health Organization will be assuming the task of translating the list into other languages, although it is anticipated that advice will be sought to corroborate CHD-related terms and definitions in other languages from the International Nomenclature Society, whose members speak a wide variety of languages. Of note, Latin terms have largely been translated into English, and are almost never used as a primary term. The Latin version of a term has by and large been listed as a synonym (e.g. "Common arterial trunk" $=$ "Truncus arteriosus" $=09.01 .01)$.

\section{Right and left}

When discussing cardiac chambers, such as atriums and ventricles, and spatial relationships, the words left and right can be confusing. Rules were therefore created to provide consistency and accuracy of descriptive terms of anatomical phenotypes. For cardiac chambers, the words left and right do not imply sidedness or position but refer to morphologically left and morphologically right. Thus, left ventricle and left atrium mean the morphologically left ventricle and morphologically left atrium, respectively. If one wishes to describe the position or sidedness of a cardiac chamber, it is necessary to use terms such as left-sided ventricle. In contrast, when describing the superior caval vein, and using the adjectival prefix left or right, it is the spatial position that is being alluded to, rather than any other connection or phenotypic variation that may exist.

\section{"Other" and "Unspecified"}

The term "other", meaning "other specified", is largely eschewed in the IPCCC with the logic that if one cannot find a particular lesion in a given list one needs to go to the higher-order term to code for an entity. The term "congenital right ventricular diverticulum" exists in the IPCCC but was not included in ICD-11. If one wishes to code for this lesion from ICD-11, the higher-order term to be used will be "Right ventricular anomaly" but not "Right ventricular anomaly, other", as depending on the list from which this term was taken "Right ventricular anomaly, other" may have different meanings and content.

All terms in the nomenclature lists theoretically end in Not Otherwise Specified "(NOS)" or "(unspecified)", in that one can always create further subdivisions for virtually any diagnosis or procedure. It follows that the generic term on its own is self-explanatory, without the need for such clarifying nomenclature being affixed. Although a subtle distinction could be made to suggest that intentionally stating "unspecified" may mean that there really is no additional specification possible, it would be impossible to know whether this is the case or whether the coder or physician involved simply did not bother to code more specifically to save time and effort. These suffixes are consequently not necessary and are not used within the Foundation level listing in ICD-11. Despite advice against this by the International Nomenclature Society, nonetheless, the ICD-11 Revision Steering Group, along with the Mortality and Morbidity Topic Advisory Groups, have opted for the retention of both "Other" and "Unspecified", both designated as "residual categories", within the Mortality and Morbidity Statistics linearisation, partly because there have always been such terms in the lexicon, going right back to 1900. Consequently, the International Nomenclature Society greatly favours the use of the congenital heart list in its unabridged and unaltered form as found in the Foundation Component of ICD-11, and published in this issue of Cardiology in the Young, where such "residual" terms do not feature.

\section{Conforming to ICD standards}

The International Classification of Diseases has certain rules that caused some of the IPCCC terms to be changed very slightly when they were incorporated into ICD-11.

1. No parentheses are allowed within the list. Thus, if a term such as "Truncus Arteriosus (Common arterial trunk)" is listed as such in the IPCCC, signifying that they are identical and used interchangeably in congenital cardiology parlance, one of the terms was consigned to the status of synonym in another column of ICD-11. This was particularly the case for Latinisms.

2. The words "and" and "or" in ICD-11 are used with their meanings in formal logic, and are not found together as "and/or". Thus "and" means that both terms must be present in the condition, whereas "or" means that either one, or the other, or both terms may be present. Thus "or" equates to "and/or".

3. In ICD-11, as in the IPCCC, no eponyms are followed by a possessive apostrophe and "s". Therefore, in ICD-11, what may be known to many as Ebstein's anomaly of the tricuspid 
valve will read Ebstein anomaly of the tricuspid valve.

4. In ICD-11, the plural form of a term in parentheses - for example, "Congenital anomaly of coronary artery(ies)" - is not allowed, and thus only the singular form is identified in ICD-11, even though a given entry in IPCCC may include both the singular and plural forms of the entity.

These minor changes have not led to any fundamental changes in definitions of the terms or in the six-digit code associated with each term in the IPCCC but are noted here to explain the slight discrepancies that may be noticed between the terms in ICD-11 and the IPCCC.

\section{Extension codes, qualifiers, and modifiers}

The IPCCC contains added codes for qualifying lesions, be it for size, degree, or subtypes. In the version of the IPCCC derived from the European Paediatric Cardiac Code, these are known as "qualifiers", whereas in the version of the IPCCC derived from the International Congenital Heart Surgery Nomenclature and Database Project, these are called "modifiers". As noted above, in ICD-11, there will be the opportunity to qualify entries within the CHD list through the use of Extension Codes that are available in a separate chapter of ICD-11. Thus, a ventricular septal defect can be qualified as "small", "moderate", or "large" by using the qualifiers provided within the Extension Code chapter (chapter X). Extension codes can never be used on their own and must always be linked to a "stem" diagnosis code in both ICD-11 and the IPCCC, whereas a diagnosis may feature cluster of extension codes to describe different characteristics, such as severity and time in life; for instance, congenital aortic stenosis can be described as "severe" and "neonatal".

\section{The CHD diagnostic tree, the foundation component of ICD-11, and the morbidity and mortality linearisation in ICD-11}

It is from the Foundation Component of ICD-11 that linearisations, also now known as Tabular Lists, are constructed. This is achieved by building a specific hierarchical tabular list from selected entities, including speciality versions of ICD-11 and countryspecific modifications. Figure 3 shows the six crosssectional Topic Advisory Groups whose purpose is to ensure structural uniformity and oversee the creation of specific linearisations, such as the joint linearisation known as morbidity and mortality statistics. The Morbidity and Mortality Statistics tabular list is designed for international mandatory reporting of data for global disease-related statistics and can be considered as the ICD-11 equivalent of ICD-10 in terms of scope, targeting mostly statistical and administrative stakeholders.

The Morbidity and Mortality Statistics Linearisation is the one part of ICD-11 that will be issued in hard copy, and because of this strict content restrictions have been imposed. As a direct consequence of these restrictions, the list of 318 congenital heart terms that was submitted by the International Nomenclature Society for the Foundation Component of ICD-11 has been pared down to 94 terms in the CHD section of the Morbidity and Mortality Statistics linearisation. Nevertheless, it is the Foundation component of ICD-11 with its full complement of 318 terms for CHD that the International Nomenclature Society will be promoting internationally to serve as a diagnostic tree to be used by all communities interested in congenital cardiology, including administrators. As countries adopt ICD-11 into their healthcare systems, the International Nomenclature Society will want to ensure uniformity in data gathering by encouraging the use of the full list of CHD terms found in the Foundation component of ICD-11 and as published in this issue of Cardiology in the Young, over the use of shortened or altered lists that may be found elsewhere in ICD-11.

\section{ICD-11 maintenance and future enhancements}

In the past, the latest version of the International Classification of Diseases could not be changed until the next iteration many years later. This is not the case for ICD-11, where there will be a mechanism for enhancing the diagnostic lists as frequently as annually. Field testing of the CHD list in ICD-11 has begun. The International Nomenclature Society fully anticipates the addition of new terms, and more accurate or elaborate definitions are likely to ensue. As "keepers" of the IPCCC and the ICD-11 list of $\mathrm{CHD}$ terms, requests for changes to the list will be handled through the International Nomenclature Society through a bidirectional relationship with the World Health Organization ICD-11 team. Requests for change, known as proposals, are to be submitted online via the ICD-11 website, either by an individual or a Society, to a Proposal Platform where they will be triaged to ensure first that they are correct and complete. If the proposal requires a scientific or medical view, as opposed to an error or classification query, it will be forwarded to the newly established Medical and Scientific Advisory Committee, whose 15 to 20 members are expert clinicians in a variety of fields drawn from the previous Topic Advisory 
Groups, which were discontinued in October, 2016. The new committee fortuitously includes a member of the International Nomenclature Society. A proposal relating to congenital cardiology could then be relayed to the International Nomenclature Society for advice in its role as a specialist editorial reviewer group. If a change is recommended, the proposal is then sent to the Classification and Statistics Advisory Committee for assessment, particularly with respect to how the proposal may affect the classification structure, as well as the collection of data for mortality and morbidity statistics, before forwarding to an implementation team. Revisions to the proposal may be made at any stage to improve it, or it may be rejected. The International Nomenclature Society, therefore, continues its commitment to this long-term enterprise, even though it has achieved the goal of providing a list of congenital heart anomaly terms, with definitions and synonyms, for ICD-11.

\section{Future projects of the International Nomenclature Society}

The International Nomenclature Society has recently been tasked with a new World Health Organization project related to the ICD-11 sister classification the International Classification of Health Interventions. The last publication in this respect from the World Health Organization was in 1978, namely the International Classification of Procedures in Medicine alongside ICD-9. A revision has not since been undertaken, as it was judged to be too complex a task. Many countries who might resource this, furthermore, had already developed their own medical procedure coding system. Despite this, in 2001 a need was perceived for those countries without such a system. After a failed attempt to use the Australian Classification of Health Interventions, in 2006 a decision was made to commence a parallel project to ICD-11 to create a classification for interventions. A health intervention is here defined as "an act performed for, with or on behalf of a person or a population, whose purpose is to assess, improve, maintain, promote or modify health, functioning or health conditions". It is profession neutral, and includes interventions related to mental and allied health, nursing and public health, in addition to surgery and medical procedures. Owing to a lack of resources, progress has been slow, and specialist clinical input limited. This has now changed, and there is now engagement with clinicians from a variety of backgrounds, as well as a tight timeline with the hope of a finished product by 2020 . It is already evident that much work is to be done to encompass the needs of congenital cardiology with respect to transcatheter interventions and cardiac surgery, as well as electrophysiology. The International Nomenclature Society has, therefore, formed a new working group specifically for this task. The first meeting of this Group will take place in Miami, Florida, between 31 January and 4 February, 2018, to discuss the goals of this new mandate and how to achieve them. Other working groups of the Society will be continuing their work in an ongoing and parallel manner, including the Archiving Working Group, which is developing a virtual atlas to illustrate each of the congenital heart malformations within the ICD-11 lexicon. These will link to the definitions, and have the potential to create hyperlinks to these from the World Health Organization ICD-11 website. The Definitions Working Group will continue to define or perfect the already existing definitions of all the items in the IPCCC.

\section{Conclusions}

The latest iteration of the International Classification of Diseases of the World Health Organization (ICD-11) is due to begin its release in 2018. Through the International Nomenclature Society, experts in the field were called upon to create a list of diagnostic terms for CHD to be included in ICD-11. This list, drawn from the Society's IPCCC, was submitted to the World Health Organization in a hierarchical manner. It contains definitions and synonyms for each term further to enhance the accuracy of coding.

Although the widely used IPCCC comes in two versions, which have been crossmapped one to the other, whereby either version can be used with easy convertibility from one to the other, the so-called "Short Lists" that have been extracted from each version vary considerably in the number of terms and the actual diagnoses selected for data compilation. With the incorporation of a thorough and unique list of diagnostic terms for congenital heart malformations into the internationally used classification scheme of ICD-11, it is expected that the many societies and organisations that use various subsets of the IPCCC for the purpose of data gathering adopt the congenital heart list of ICD-11 as their "short list" for all purposes, including those related to administrative, research, and clinical work.

The International Nomenclature Society anticipates that the use of its diagnostic list for CHD in ICD-11 for future cooperative multi-institutional studies and epidemiological surveys will facilitate accurate data gathering, and ultimately enable the optimisation of the quality and effectiveness of healthcare for our patients with congenital cardiac malformations. 


\section{Acknowledgements}

The Definitions Working Group of the International Society for Nomenclature of Paediatric and Congenital Heart Disease wishes to acknowledge Rodney Franklin, chief liaison between the Nomenclature Society and the World Health Organization, and chairpersons Steven Colan and Henry (Hal) Walters, for their steadfast work and leadership since the inception of the ICD-11 CHD project.

\section{Financial Support}

The Definitions Working Group gratefully acknowledges the "Heart of a Child, Michigan" through the Children's Hospital of Michigan Foundation, and "Angela's Big Heart for Little Kids Fund" through the Montreal Children's Hospital Foundation, for donations given in support of the ICD-11 CHD Nomenclature project.

\section{Conflicts of Interest}

None.

\section{References}

1. Smith R. All changed, changed utterly. Br Med J 1998; 316: 1917-1918.

2. Learning from Bristol: the report of the public inquiry into children's heart surgery at the Bristol Royal Infirmary 1984-1995. Retrieved October 14, 2013, from http://webarchive.nationalarc hives.gov.uk/+/www.dh.gov.uk/en/Publicationsandstatistics/ Publications/PublicationsPolicyAndGuidance/DH_4005620.

3. Wright J. Putting a surgeon under: a personal story of hospital politics. Retrieved October 14, 2013, from http://www.uow.edu. $\mathrm{au} / \mathrm{arts} / \mathrm{sts} / \mathrm{bmartin} /$ dissent/documents/Wright.

4. The Report of the Manitoba Pediatric Cardiac Surgery Inquest: An Inquest into twelve deaths at the Winnipeg Health Sciences Centre in 1994. Retrieved October 14, 2013 from http://www.pedia triccardiacinquest.mb.ca/.

5. Sherry A. Some Surgery Halted at Children's. Denver Post, 2001. Retrieved September 8, 2017, from http://extras.denverpost.com/ news/news0228a.htm.

6. Sherry A. Children's Hospital cardiology chief told to resign. Denver Post, 2001. Retrieved September 8, 2017, from http:// extras.denverpost.com/news/news $0301 \mathrm{~b} . \mathrm{htm}$.

7. Retrieved September 10, 2017, from http://edition.cnn.com/ 2013/08/12/health/kentucky-children-update/index.html.

8. Franklin RC, Beland MJ, Krogmann ON. Mapping and coding of nomenclatures for paediatric and congenital heart disease. Cardiol Young 2006; 16: 105-106.

9. Brown KL, Crowe S, Franklin RC, et al. Trends in 30-day mortality rate and case mix for paediatric cardiac surgery in the UK between 2000 and 2010. Open Heart 2015; 2: e000157.

10. Jacobs JP, Mayer JE Jr, Mavroudis C, et al. The Society of Thoracic Surgeons Congenital Heart Surgery Database: 2016 Update on Outcomes and Quality. Ann Thorac Surg 2016; 101: 850-862.

11. Jacobs JP, O’Brien SM, Pasquali SK, et al. The Society of Thoracic Surgeons Congenital Heart Surgery Database Mortality Risk Model: Part 2-clinical application. Ann Thorac Surg. 2015; 100: 1063-1070.
12. Jacobs JP, Mayer JE Jr, Mavroudis C, et al. The Society of Thoracic Surgeons Congenital Heart Surgery Database: 2017 Update on Outcomes and Quality. Ann Thorac Surg 2017; 103: 699-709.

13. World Health Organization. History of the development of the ICD. Retrieved September 20, 2017, from http://www.who.int/ classifications/icd/en/HistoryOfICD.pdf.

14. Greenwood M. Medical statistics from Graunt to Farr. Cambridge University Press, Cambridge, 1948.

15. Farr W. First annual report. Registrar General of England and Wales, London, 1839: 99.

16. Retrieved September 24, 2017, from http://www.wolfbane. $\mathrm{com} / \mathrm{icd} /$.

17. Farr W. Sixteenth annual report. Registrar General of England and Wales, London, 1856: 75

18. Nightingale F. Proposals for a Uniform Plan of Hospital Statistics. Fourth International Statistical Congress, London, 1860.

19. Retrieved September 23, 2017, from http://www.who.int/ classifications/en/.

20. Franklin RC, Jacobs JP, Krogmann ON, et al. Nomenclature for congenital and paediatric cardiac disease: historical perspectives and The International Pediatric and Congenital Cardiac Code. Cardiol Young 2008; 18 (Suppl 2): 70-80.

21. Franklin RCG, Anderson RH, Daniëls O, et al. The European Paediatric Cardiac Code. Cardiol Young 1999; 9: 633-657.

22. Association for European Paediatric Cardiology. The European Paediatric Cardiac Code. Cardiol Young 2000; 10 (Suppl 1): $1-146$.

23. Mavroudis C, Jacobs JP. Congenital heart surgery nomenclature and database project: overview and minimum dataset. Ann Thorac Surg 2000; 69: S2-S17.

24. Mavroudis C, Jacobs JP. (eds). Congenital Heart Surgery Nomenclature and Database Project. Ann Thorac Surg 2000: 69 Suppl: S1-S372.

25. Béland M, Jacobs JP, Tchervenkov CI, Franklin RCG. The International Nomenclature Project for Paediatric and Congenital Heart Disease: Report from the Executive of The International Working Group for Mapping and Coding of Nomenclatures for Paediatric and Congenital Heart Disease. Cardiol Young 2002; 12: 425-430.

26. Franklin RCG, Jacobs JP, Tchervenkov CI, Béland MJ. The International Nomenclature Project for Congenital Heart Disease: bidirectional crossmap of the short lists of the European Paediatric Cardiac Code and the International Congenital Heart Surgery Nomenclature Database Project. Cardiol Young 2002; 12: 431-435.

27. Béland MJ, Franklin RCG, Jacobs JP, et al. Update from The International Working Group for Mapping and Coding of Nomenclatures for Paediatric and Congenital Heart Disease. Cardiol Young 2004; 14: 225-229.

28. O'Brien SM, Clarke DR, Jacobs JP, et al. An empirically based tool for analyzing mortality associated with congenital heart surgery. J Thorac Cardiovasc Surg 2009; 138: 1139-1153.

29. Crowe S, Brown KL, Pagel C, et al. Development of a diagnosisand procedure-based risk model for 30-day outcome after pediatric cardiac surgery. J Thoracic Cardiovasc Surg 2013; 145: 1270-1278.

30. Rogers L, Brown KL, Franklin RC, et al. Improving risk adjustment for mortality after pediatric cardiac surgery: The UK PRAiS 2 model. Ann Thorac Surg 2017; 104: 211-219.

31. World Health Organization. Basic Documents, 47th ed. World Health Organization, Geneva, 2009.

32. Tudorache T, Falconer S, Nyulas $\mathrm{C}$, et al. Supporting the Collaborative Authoring of ICD-11 with WebProtégé. AMIA Annu Symp Proc 2010; 2010: 802-806.

33. Retrieved September 23, 2017, from http://www.snomed.org/ snomed-ct. 
34. Cronk CE, Malloy ME, Pelech AN, et al. Completeness of state administrative databases for surveillance of congenital heart disease. Birth Defects Res A Clin Mol Teratol 2003; 67: 597-603.

35. Frohnert BK, Lussky RC, Alms MA, Mendelsohn NJ, Symonik DM, Falken MC. Validity of hospital discharge data for identifying infants with cardiac defects. J Perinatol 2005; 25: 737-742.

36. Strickland MJ, Riehle-Colarusso TJ, Jacobs JP, et al. The importance of nomenclature for congenital heart disease: implications for research and evaluation. Cardiol Young 2008; 18 (Suppl 2): 92-100.
37. Pasquali SK, Peterson ED, Jacobs JP, et al. Differential case ascertainment in clinical registry versus administrative data and impact on outcomes assessment for pediatric cardiac operations. Ann Thorac Surg 2013; 95: 197-203.

38. Pasquali SK, He X, Jacobs JP, et al. Measuring hospital performance in congenital heart surgery: administrative vs. clinical registry data. Ann Thorac Surg 2015; 99: 9332-9338.

39. Congenital cardiology listing in Foundation Component of ICD-11. Retrieved November 12, 2017, from https://icd.who.int/ dev11/f/en\#/http $\% 3 a \% 2$ f $\% 2$ fid.who.int $\% 2$ ficd $\% 2$ fentity $\% 2 f 183$ 3285474 . 
Table 1. Systematic hierarchical list of congenital cardiology terms submitted to the World Health Organization for inclusion in the Foundation Component of the 11th revision of the International Classification of Diseases. ${ }^{3}$

\begin{tabular}{llllll}
\hline Level 0 & Level I & Level II & Level III & Level IV & Level V \\
\hline
\end{tabular}

Level VII

development

anomaly of heart and

great vessels

Congenital anomaly of position
or spatial relationships of

or spatial relationships of
thoraco-abdominal organs

Anomalous position-

orientation of heart

Laevocardia

Dextrocardia

Mesocardia

Usual atrial arrangement

Abnormal atrial arrangement

Atrial situs inversus

somerism of right atrial appendages

Abnormal ventricular

relationships

somerism of left atrial appendages

Right hand pattern ventricular

topology

Left hand pattern ventricular

topology

Crisscross heart

uperior-inferior ventricular

Abnormal relationship of great

arterial roots

Aortic root directly anterior to

pulmonary root

Aortic root anterior and rightward

to pulmonary root

Aortic root anterior and leftward to

pulmonary root

Aortic root side by side and directly

rightward to pulmonary root

Aortic root side by side and directly

Aortic root directly posterior to

pulmonary root

Aortic root posterior and rightward

to pulmonary root

Aortic root posterior and leftward to

Abnormal intrapericardial

pulmonary root

course of great

Spiralling course of great arteries

Parallel course of great arteries

Right isomerism

Left isomerism 
Double outlet right ventricle wit

without pulmonary stenosis

Double outlet left ventricle

intact ventricular septum

Common arterial trunk

Common arterial trunk with aortic dominance

\section{Congenital anomaly of} mediastinal vein
Congenital anomaly of mediastinal systemic vein

\section{Congenital anomaly of superio$$
\text { omaly of superior }
$$
caval vein

Common arterial trunk with

pulmonary dominance and aortic

Congenital truncal valva

regurgitation

Congenital truncal valvar stenosi

Dysplasia of truncal valve

bsent right superior caval ve

Left superior caval

$$
\begin{aligned}
& \text { Left superior caval vein to } \\
& \text { coronary sinus } \\
& \text { Left superior caval vein to } \\
& \text { left-sided atrium }
\end{aligned}
$$

Congenital anomaly of inferio

$$
\text { caval vein }
$$

Interrupted inferior caval vein with absent suprarenal

segment and azygo

Congenital anomaly of coronary

Coronary sinus orifice atresia or

Anomalous hepatic venous stenosis 


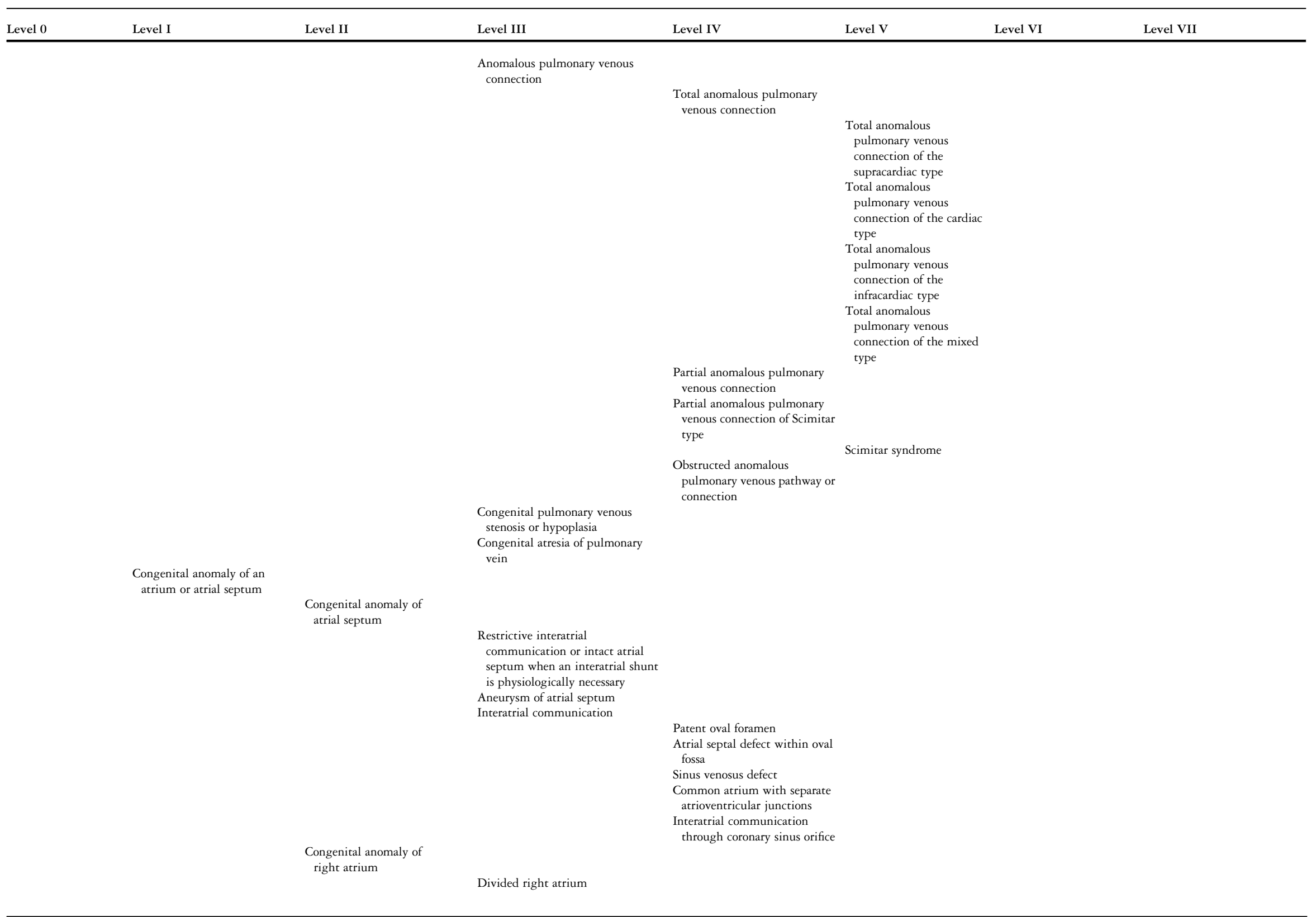


Congenital anomaly of

left atrium

Divided left atrium

Right-sided juxtaposition of the

atrial appendages

Congenital anomaly of a atrioventricular valve ular septum

Congenital anomaly of tricuspid valve

Congenital tricuspid regurgitation Congenital tricuspid valvar stenosis Tricuspid annular hypoplas

Dysplasia of tricuspid valve

Straddling tricuspid valve

Overriding tricuspid valve
Ebstein malformation of tricuspid

valve

Congenital anomaly of mitral

valve

Congenital mitral regurgitation

Congenital mitral valvar stenosis

Mitral annular hypoplasi

traddling mitral valve

Overriding mitral valve

Dysplasia of mitral valve
Supravalvar or intravalvar mitral

Supravalvar or intravalvar mitral
ring

Congenital mitral valvar prolapse rue cleft of anterior mitral le

Congenital anomaly of
subvalvar apparatus

Congenital mitral subvalvar

stenosis
Parachute malformation of

mitral valve

Congenital anomaly of leftsided atrioventricular valve sided atrioventricular valve
in double inlet ventricle Congenital anomaly of rightsided atrioventricular valve in double inlet ventricle Common atrioventricula

junctio

Atrioventricular septal defect

Atrioventricular septal defect with balanced ventricles Atrioventricular septal defect with ventricular imbalance

Atrioventricular septal

defect with ventricul

imbalance with

dominant right ventricle,

hypoplastic left ventricle

Atrioventricular septal

defect with ventricula

imbalance with

dominant left ventricle, 
Right ventricular hypoplasia

Congenital right ventricular outflow

tract obstruction

Double-chambered right ventricle

Right ventricular myocardial

sinusoids

Parchment right ventricle

(including Uhl anomaly)

Tetralogy of Fallot

Tetralogy of Fallot with absent

pulmonary valve syndrom

Tetralogy of Fallot wi

pulmonary atresia

Tetralogy of Fallot with

pulmonary atresia and

collateral artery

Congenital left ventricular

anomaly

Left ventricular hypoplasia

Congenital left ventricular

aneurysm or diverticulum

Congenital left ven

Left ventricular outflow tract

obstruction due to

Left heart obstruction at multiple

sites

atrioventricular valve

Restrictive interventricular

communication when an

interventricular shunt is

Vhysiologically necessary

Perimembranous central

ventricular septal defect

Inlet ventricular septal defect

without a common

Inlet perimembranous

ventricular septal defect without atrioventricular septal malalignment without a common atrioventricular junction Inlet perimembranous ventricular septal defect with atrioventricular septal malalignment and 
Doubly committed juxta-

arterial ventricular septa

defect with anteriorly

malaligned fibrous outlet

septum and muscular postero-

inferior rim

Doubly committed juxta-

arterial ventricular sept

defect with anteriorly

malaligned fibrous outlet
septum and perimembranous

Outlet muscular

ventricular septal defect with posteriorly

$$
\begin{aligned}
& \text { malaligne } \\
& \text { septum }
\end{aligned}
$$

septum

ventricular septal defect
vet perimembranous with posteriorly

malaligned outle

septum

Doubly committed juxta-

arterial ventricular septal

defect with posteriorly

malaligned fibrous outlet

septum

Doubly committed juxtaarterial ventricular septal defect with posteriorly

malaligned fibrous outlet septum and muscular posteroinferior rim

Doubly committed juxta-

arterial ventricular septal

defect with posteriorly

malaligned fibrous outlet

septum and perimembranous

extension

Functionally univentricular

Double inlet atrioventricular

connection

Double inlet right ventricle

Double inlet to solitary ventricle of

indeterminate morphology 


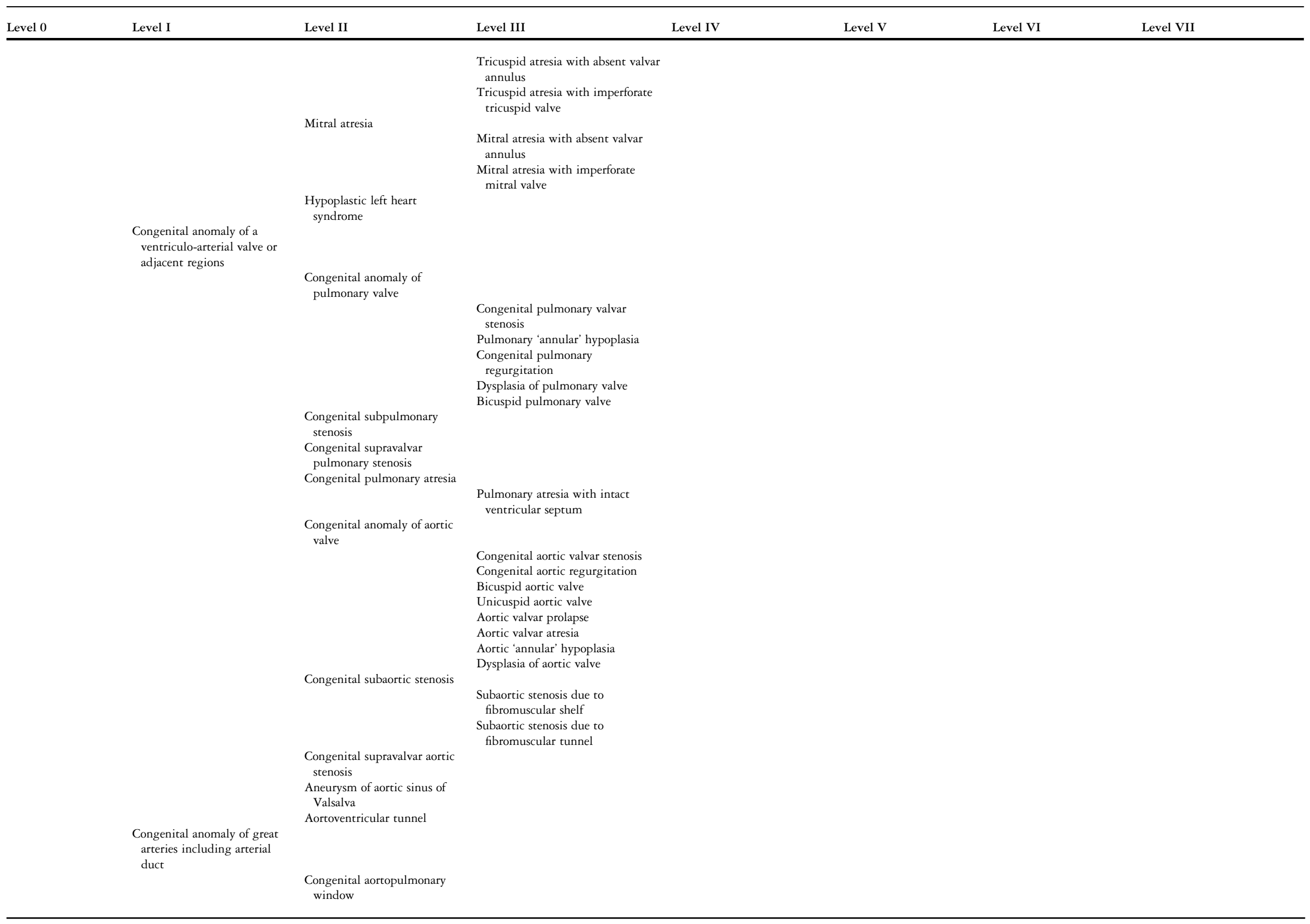




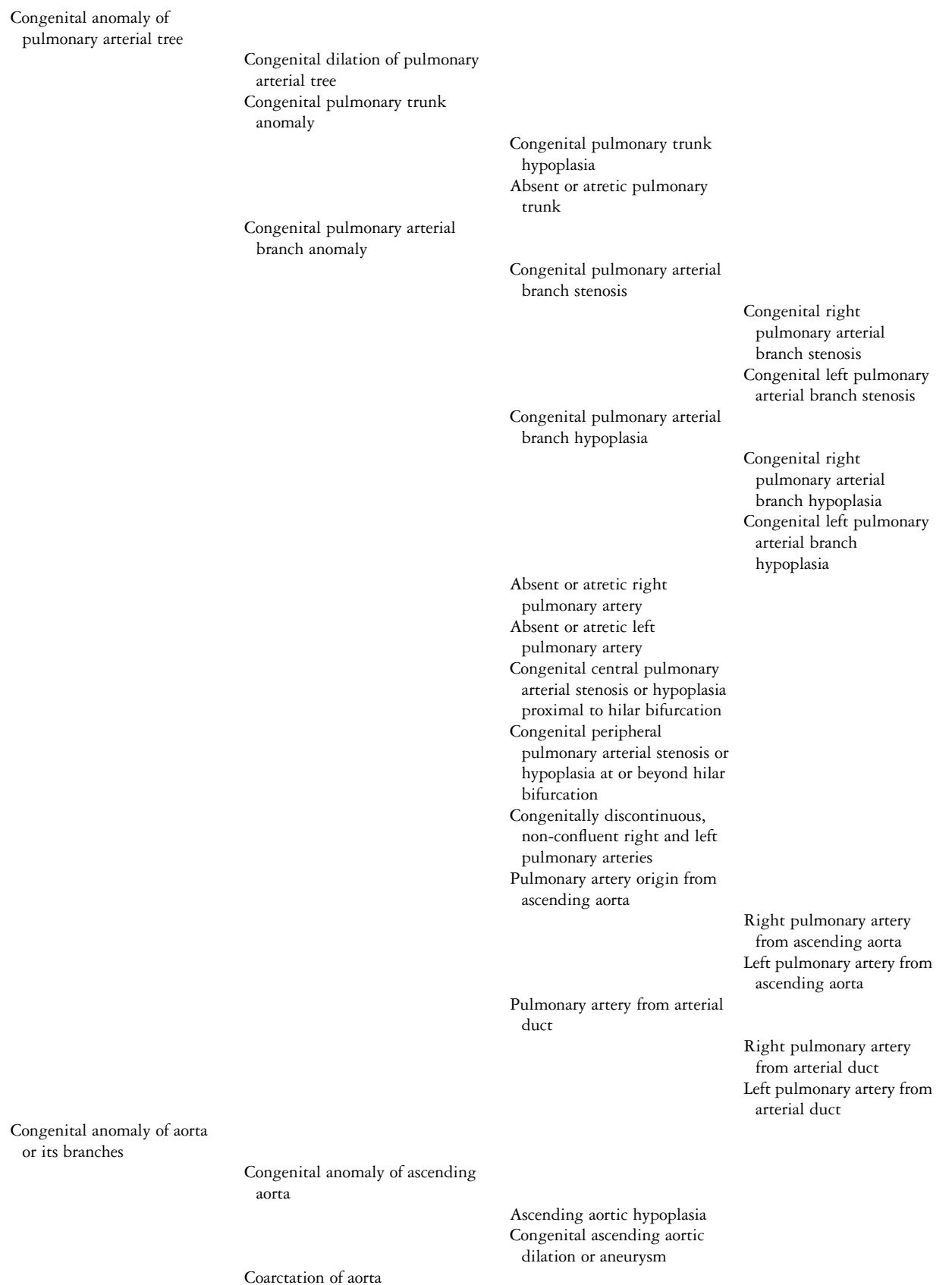




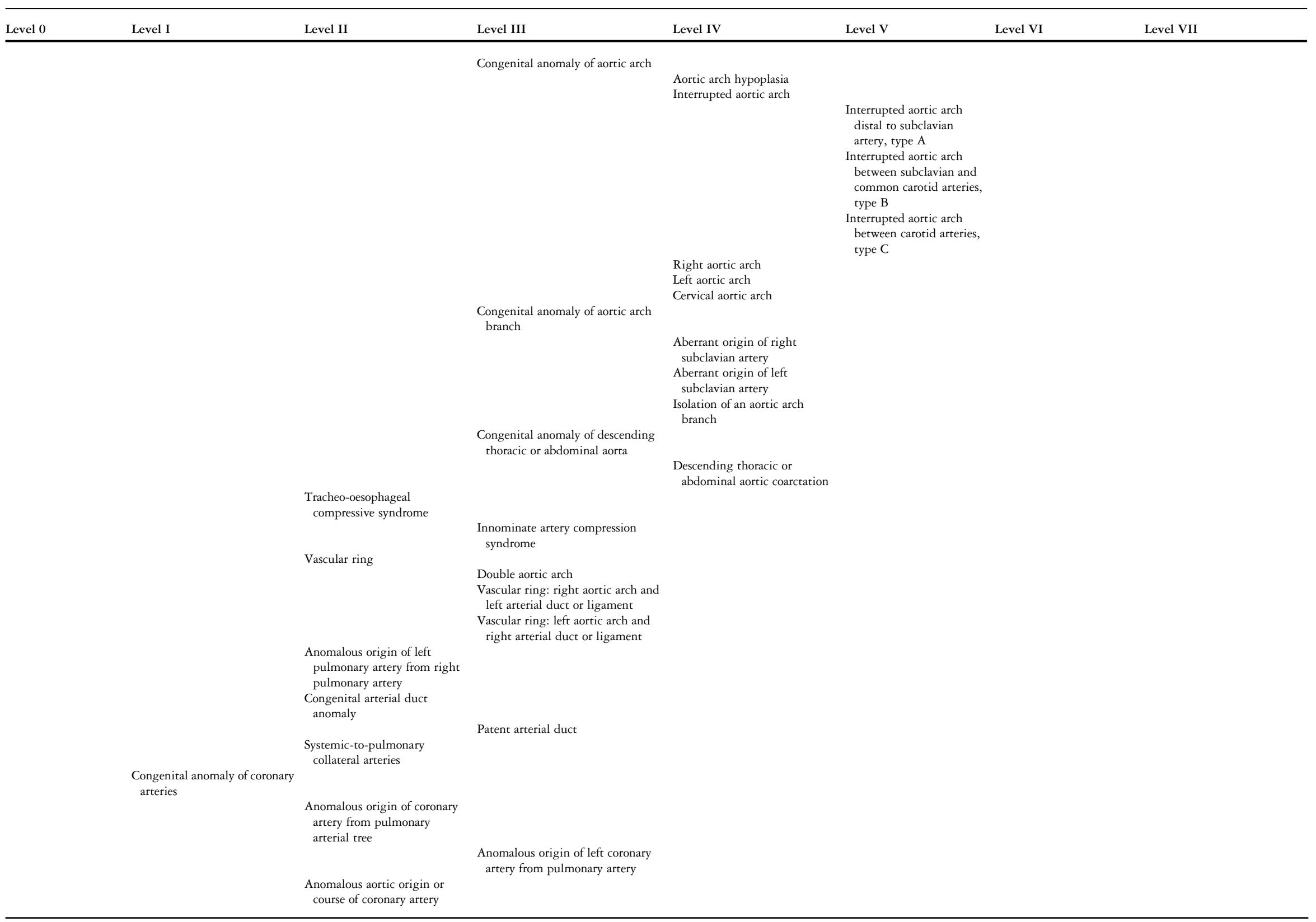


Anomalous aortic origin of coronary

artery with ventriculo-arterial

Right coronary artery from left

ortic sinus with ventriculo-

arterial concordance

Left coronary artery from right

artic sinus with ventriculo-

Anterior descending from righ

arterial concordance

coronary attery across right

Intramural proximal coronary

arterial course

Myocardial bridging of heart

coronary artery

Congenital coronary arteria

orifice stenosis

orifice atresia

Congenital coronary arteria

fistula

Congenital coronary arterial fistul to right ventricle

Congenital coronary arterial fistul

to left ventricle

Congenital coronary arteria

aneurysm

Congenital pericardia

anomaly

Pulmonary 
Table 2. Systematic list of congenital cardiology terms submitted to the World Health Organization for inclusion in the Foundation Component of the 11th revision of the International Classification of Diseases with their definitions and acceptable synonyms, ${ }^{39}$ and their IPCCC six digit numbers.

ICD-11 congenital cardiology terms with

International Paediatric and Congenital

Cardiac Code (IPCCC) numbers, definitions and synonyms

IPCCC code Definition with commentary

Synonyms

Structural developmental anomaly of heart and great vessels

Congenital anomaly of position or spatial relationships of thoraco-abdominal organs

Anomalous position-orientation of heart

01.01 .59

03.01 .13

02.01 .09

02.01 .03

Laevocardia

Dextrocardia

02.01 .02

Mesocardia

Extrathoracic heart

Usual atrial arrangement

Abnormal atrial arrangement

Atrial situs inversus

Isomerism of right atrial appendages

Isomerism of left atrial appendages

Abnormal ventricular relationships

Right hand pattern ventricular topology
A congenital malformation of the heart and-or great vessels

A congenital cardiovascular finding or malformation associated with an abnormal position of the heart or thoraco-abdominal organs, or an abnormal relative position of its component parts

A congenital cardiovascular finding/malformation in which there Malposition of heart

is an abnormality of the position or orientation of heart

A congenital cardiovascular finding in which the heart is

Levocardia; left-sided heart

predominantly to the left of the thoracic midline.

This term should be coded only in the context of complex heart disease.

This is independent of the orientation of the cardiac apex

A congenital cardiovascular malformation in which the heart is Right-sided heart; heart in right chest; congenital dextrocardia predominantly to the right of the thoracic midline. of heart

This is independent of the orientation of the cardiac apex

02.01.04 A congenital cardiovascular malformation in which the heart is Midline heart central or midline within the thorax

02.01.01 A congenital cardiovascular malformation in which the heart is Ectopia cordis at least partially outside of the thorax

01.03.00 A congenital cardiac finding in which the atrial laterality Atrial situs solitus

(sidedness) is normal.

This is a normal finding that should be coded only in the context of complex heart disease

01.03.06 A congenital cardiac malformation in which there is an Abnormal atrial situs

abnormality of the laterality (or sidedness) of the atria

01.03.01 A congenital cardiac malformation in which the atrial Mirror-image atrial arrangement

morphologies and positions are the mirror image of normal

01.03.02 A congenital cardiac malformation in which both atrial
appendages have the morphology of a right atrial appendage

01.03.03 A congenital cardiac malformation in which both atrial Isomeric left atrial appendages

appendages have the morphology of a left atrial appendage

02.04.12 A congenital cardiac malformation in which the ventricular

positions relative to each other or their laterality (sidedness) are abnormal

02.03.01 A congenital cardiac finding in which the chirality, or handedness, D-loop ventricles; Dextro-ventricular looping; of the ventricles is normal, also known as D-loop.

D-bulboventricular loop

Chirality or handedness with a right-handed right ventricle mean

likening the morphological right ventricle to a right hand by

representing the inflow by the thumb, outflow by the index finger, and

septum by the palm. This is a normal finding but should be coded in the

Isomeric right atrial appendages 
Crisscross heart (twisted atrioventricular connections)

Superior-inferior ventricular relationship

02.03 .03

Abnormal relationship of great arterial roots

02.06 .12

Aortic root directly anterior to pulmonary root

Aortic root anterior and rightward to pulmonary 02.06 .02 root

Aortic root anterior and leftward to pulmonary

02.06 .04

Aortic root side by side and directly rightward to 02.06 .01 pulmonary root

Aortic root side by side and directly leftward to 02.06 .05 pulmonary roo

Aortic root directly posterior to pulmonary root

02.06 .07

Aortic root posterior and rightward to pulmonary 02.06 .00 root

Aortic root posterior and leftward to pulmonary 02.06 .06 roo

Abnormal intrapericardial course of great arteries

02.07 .0 presence of abnormal positions of the atria or great arteries where it represents an abnormality of the interrelationships of the ventricles relative to the remainder of the heart

A congenital cardiac malformation in which the chirality, or

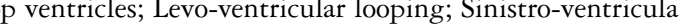
known as L-loop

A congenital cardiac malformation in which the atrioventricular Twisted atrioventricular connections inflow vectors are approximately orthogonal or perpendicular A congenital cardiac malformation in which the ventricles are positioned supero-inferior to each other.

Excludes situations where one ventricle does not receive an atrioventricular valve (univentricular atrioventricular connection)

A congenital cardiovascular malformation in which the aortic root or its remnant is abnormally positioned relative to the pulmonary root or its remnant

A congenital cardiovascular malformation in which the aortic root or its remnant is positioned directly anterior to the pulmonary root or its remnant

A congenital cardiovascular malformation in which the aortic root or its remnant is positioned anterior and to the right of the pulmonary root or its remnant

A congenital cardiovascular malformation in which the aortic root or its remnant is positioned anterior and to the left of the pulmonary root or its remnant.

A congenital cardiovascular malformation in which the aortic root or its remnant is positioned directly to the right of the pulmonary root or its remnant

A congenital cardiovascular malformation in which the aortic root or its remnant is positioned directly to the left of the pulmonary root or its remnant

A congenital cardiovascular malformation in which the aortic root or its remnant is positioned directly posterior to the pulmonary root or its remnant

A congenital cardiovascular finding in which the aortic root or it remnant is positioned posterior and to the right of the pulmonary root or its remnant

A congenital cardiovascular malformation in which the aortic root or its remnant is positioned posterior and to the left of the pulmonary root or its remnant.

This is a normal finding but should only be coded in the context of complex CHD.

A congenital cardiovascular malformation in which the course of the ascending aorta or its remnant is abnormal relative to the course of the pulmonary trunk or its remnant.

The normal course of the proximal great vessels results in a nearly orthogonal relationship of the axis of the aorta to the axis of the pulmonary artery

Upstairs-downstairs ventricular relationship; supero-inferior heart

Abnormal relationships of great arteries; abnormal relationships of great vessels

Antero-posterior great arteries; antero-posterior great vessels; A-malposed aorta; A-malposed great arteries; aorta directly anterior to pulmonary artery

D-malposed great arteries with anterior aorta; D-malposed great vessels with anterior aorta; Dextroposed great arteries with anterior aorta; Dextroposed great vessels with anterior aorta L-malposed great arteries with anterior aorta; L-malposed great vessels with anterior aorta; levoposed great arteries with anterior aorta; levoposed great vessels with anterior aorta

D-malposed great arteries; D-malposed great vessels; dextroposed great arteries; dextroposed great vessels

L-malposed great arteries; L-malposed great vessels; levoposed great arteries; levoposed great vessels

Segmental analysis $\mathrm{D}$ with posterior aorta; normally related great arteries; normally related great vessels; D-malposed great arteries with posterior aorta; D-malposed great vessels with posterior aorta

-malposed great arteries with posterior aorta; L-malposed great vessels with posterior aorta; levoposed great arteries with posterior aorta; levoposed great vessels with posterior aorta; mirror imaged normally related great arteries; mirror imaged normally related great vessels 
ICD-11 congenital cardiology terms with

International Paediatric and Congenital

Cardiac Code (IPCCC) numbers, definitions

and synonyms

Spiralling course of great arteries

02.07 .00

$$
\text { A conger }
$$
axis of the ascending aorta to the axis of the pulmonary trunk. This is a normal finding but should be coded in the presence of abnormal positions of the great arteries relative to the ventricles

Parallel course of great arteries

Visceral heterotaxy

congenital cardiovascular finding in which the course of the great arteries results in a nearly parallel relationship of the axis of the ascending aorta to the axis of the pulmonary trunk

A congenital malformation in which the internal thoraco-

abdominal organs demonstrate abnormal arrangement across the left-right axis of the body. By convention, heterotaxy syndrome does not include patients with complete mirrorimaged arrangement of the internal organs along the leftright axis also known as "situs inversus totalis"

Right isomerism

03.01 .04

A congenital cardiovascular malformation that is a variant of heterotaxy syndrome in which some paired structures on opposite sides of the left-right axis of the body are symmetrical mirror images of each other, and have the

morphology of the normal right-sided structures

Left isomerism

03.01 .05

Total mirror imagery

03.01 .03

Congenital anomaly of an atrioventricular or

01.03 .09 ventriculo-arterial connection

Concordant atrioventricular connections

01.04 .00 heterotaxy syndrome in which some paired structures on opposite sides of the left-right axis of the body are

symmetrical mirror images of each other, and have the morphology of the normal left-sided structures right axis of the body.

A congenital cardiovascular malformation in which one or more of the following connections is abnormal: 1) the morphologically right atrium to the morphologically right ventricle; 2) the morphologically left atrium to the morphologically left ventricle; 3 ) the morphologically right ventricle to the pulmonary trunk; 4) the morphologically left ventricle to the aorta.

This excludes codes for hearts with a univentricular atrioventricular connection (mitral atresia, tricuspid atresia and double inlet ventricle), as these are listed under Functionally Univentricular Heart

A congenital cardiovascular finding in which the congenital cardiovascular finding in which the course of the

Normal course of great arteries

Abnormal arrangement of thoraco-abdominal organs; situs

ambiguus; heterotaxy; heterotaxy syndrome; heterotaxia;

heterotaxia syndrome; splenic syndromes

Asplenia syndrome; bilateral right sidedness; Ivemark syndrome; splenic agenesis syndrome

A congenital cardiovascular malformation that is a variant of an Polysplenia syndrome; bilateral left sidedness; Moller syndrome

A congenital malformation in which there is complete mirror- Situs inversus totalis; situs inversus viscerum; situs inversus or imaged arrangement of the internal organs along the left- transversus; visceral inversion morphologically right atrium connects to the morphologically right ventricle and the morphologically left atrium connects to the morphologically left ventricle. left atrium connects to the morphologically left ventricle.
This is a normal finding that should only be coded when associated with abnormal atrial arrangement and/or ventriculo-arterial connections
Normal atrioventricular connections; atrioventricular concordance 

arteries

Transposition of the great arteries with concordant 01.01 .02 atrioventricular connections and intact ventricular septum

Transposition of the great arteries with concordant 01.01.10 atrioventricular connections and ventricular septal defect

Transposition of the great arteries with concordant 01.01 .10 atrioventricular connections and ventricular $+07.09 .01$

septal defect and left ventricular outflow tract obstruction

Concordant ventriculo-arterial connections

01.05 .00

Concordant ventriculo-arterial connections with

01.05 .10 parallel great arteries
A congenital cardiac malformation in which the morphologically Atrioventricular discordance right atrium connects to the morphologically left ventricle and the morphologically left atrium connects to the morphologically right ventricle

A congenital cardiovascular malformation in which the morphologically right atrium connects to the morphologically left ventricle, the morphologically left atrium connects to the morphologically right ventricle, the morphologically right ventricle connects to the aorta, and the morphologically left ventricle connects to the pulmonary trunk

A congenital cardiovascular malformation in which the morphologically right ventricle connects to the aorta and the morphologically left ventricle connects to the pulmonary trunk

A congenital cardiovascular malformation in which the morphologically right atrium connects to the morphologically right ventricle, the morphologically left atrium connects to the morphologically left ventricle, the morphologically right ventricle connects to the aorta, the morphologically left ventricle connects to the pulmonary trunk, and a ventricular septal defect is not present

A congenital cardiovascular malformation in which the morphologically right atrium connects to the morphologically right ventricle, the morphologically left atrium connects to the morphologically left ventricle, the morphologically right ventricle connects to the aorta, the morphologically left ventricle connects to the pulmonary trunk, and one or more ventricular septal defects are present

A congenital cardiovascular malformation in which the morphologically right atrium connects to the morphologically right ventricle, the morphologically left atrium connects to the morphologically left ventricle, the morphologically right ventricle connects to the aorta, the morphologically left ventricle connects to the pulmonary trunk, one or more ventricular septal defects are present, and left ventricular outflow tract obstruction is present

A congenital cardiovascular finding in which the left ventricle is Concordant ventriculo-arterial alignments connected to the aorta or its remnant and the right ventricle is connected to the pulmonary trunk or its remnant.

This is a normal finding that should only be coded when associated with abnormal atrioventricular connections and/or ventriculoarterial arrangement

A congenital cardiovascular malformation in which the left ventricle is connected by way of an infundibulum to the aorta or its remnant, the right ventricle is connected to the pulmonary trunk or its remnant, and the proximal arteries have a parallel course
Discordant atrioventricular and ventriculo-arterial connections; transposition $\{\mathrm{S}, \mathrm{L}, \mathrm{L}\}$; transposition $\{\mathrm{I}, \mathrm{D}, \mathrm{D}\}$; corrected transposition of the great arteries; corrected transposition of the great vessels; congenitally corrected transposition of the great vessels; double discordance; physiologically corrected transposition of the great vessels; physiologically corrected transposition of the great arteries

Discordant ventriculo-arterial connections; transposition of the great vessels; complete transposition of great vessels

Transposition $\{\mathrm{S}, \mathrm{D}, \mathrm{D}\}$; transposition $\{\mathrm{I}, \mathrm{L}, \mathrm{L}\}$; concordan atrioventricular and discordant ventriculo-arterial connection with intact ventricular septum; simple transposition

Concordant atrioventricular connections and discordant ventriculo-arterial connections with ventricular septal defect

Concordant atrioventricular connections and discordant ventriculo-arterial connections with ventricular septal defect and left ventricular outflow tract obstruction; transposition with ventricular septal defect and pulmonary stenosis

Anatomically corrected malposition; anatomically corrected malposition 
ICD-11 congenital cardiology terms with

International Paediatric and Congenital

Cardiac Code (IPCCC) numbers, definitions and synonyms

Double outlet right ventricle

A congenital cardiovascular malformation in which both great arteries arise entirely or predominantly from the morphologically right ventricle

Double outlet right ventricle with subaortic or doubly committed ventricular septal defect and

pulmonary stenosis, Fallot type

Double outlet right ventricle with subaortic ventricular septal defect and pulmonary stenosis, +07.13 .04$ Fallot type

Double outlet right ventricle with doubly committed ventricular septal defect and pulmonary stenosis, Fallot type

Double outlet right ventricle with subpulmonary $\quad 01.01 .18$ ventricular septal defect, transposition type

Double outlet right ventricle with non-committed 01.01.19 ventricular septal defect

Double outlet right ventricle with subaortic or doubly committed ventricular septal defect without pulmonary stenosis, ventricular septal defect type

Double outlet right ventricle with subaortic ventricular septal defect without pulmonary stenosis

Double outlet right ventricle with doubly committed ventricular septal defect without pulmonary stenosis
01.01 .40

A congenital cardiovascular malformation that is a variant of double outlet right ventricle with concordant atrioventricula connections, a subaortic or doubly committed (with absence or deficiency of the conal septum) ventricular septal defect, and pulmonary outflow tract obstruction

A congenital cardiovascular malformation that is a variant of double outlet right ventricle with concordant

atrioventricular connections, a subaortic ventricular septa defect, and pulmonary outflow tract obstruction

A congenital cardiovascular malformation that is a variant of double outlet right ventricle with concordant atrioventricular connections, a doubly committed ventricular septal defect with absence or deficiency of the conal septum, and pulmonary outflow tract obstruction

A congenital cardiovascular malformation that is a variant of

double outlet right ventricle with concordant atrioventricula connections that is associated with a subpulmonary ventricular septal defect (includes Taussig-Bing heart)

A congenital cardiovascular malformation that is a variant of double outlet right ventricle with concordant atrioventricular connections that is associated with ventricular septal defect that is remote from the ventricular outflow tracts and usually within the inlet or muscular septum

A congenital cardiovascular malformation that is a variant of double outlet right ventricle with concordant atrioventricular connections, a subaortic or doubly committed (with absence or deficiency of the conal septum) ventricular septal defect, and unobstructed pulmonary outflow tract

01.01 .40 $+07.13 .04$

A congenital cardiovascular malformation that is a variant of double outlet right ventricle with concordant atrioventricular connections, a subaortic ventricular septal defect, and unobstructed pulmonary outflow tract

01.01.40 A congenital cardiovascular malformation that is a variant of

+07.13 .02 double outlet right ventricle with concordant atrioventricular connections, a doubly committed ventricular septal defect with absence or deficiency of the conal septum, and unobstructed pulmonary outflow tract
Double outlet right ventricle with subaortic or doubly committed interventricular communication and pulmonary stenosis, Fallot type

Double outlet right ventricle with subaortic interventricular communication and pulmonary stenosis, Fallot type

Double outlet right ventricle with doubly committed interventricular communication and pulmonary stenosis, Fallot type

Double outlet right ventricle with subpulmonary

interventricular communication, transposition type; Taussig-

Bing syndrome; Taussig-Bing malformation; Taussig-Bing heart

Double outlet right ventricle with remote ventricular septa defect; Double outlet right ventricle with uncommitted ventricular septal defect; double outlet right ventricle with non-committed interventricular communication

Double outlet right ventricle with subaortic or doubly committed interventricular communication without pulmonary stenosis, ventricular septal defect type

Double outlet right ventricle with subaortic interventricular communication without pulmonary stenosis, ventricular septal defect type

Double outlet right ventricle with doubly committed interventricular communication without pulmonary stenosis, ventricular septal defect type 
Double outlet right ventricle with intact ventricular septum

Double outlet left ventricle

Common arterial trunk

Common arterial trunk with aortic dominance

09.01 .15

Common arterial trunk with aortic dominance and 09.01.14 both pulmonary arteries arising from trunk

Common arterial trunk with aortic dominance and 09.01.11 one pulmonary artery absent from trunk, isolated pulmonary artery

Common arterial trunk with pulmonary dominance and aortic arch obstruction

Common arterial trunk with pulmonary dominance and interrupted aortic arch

Common arterial trunk with pulmonary dominance and aortic coarctation

Congenital truncal valvar regurgitation

Congenital truncal valvar stenosis

Dysplasia of truncal valve

Congenital anomaly of mediastinal vein

09.01 .12
A congenital cardiovascular malformation that is a variant of double outlet right ventricle that is associated with an intact ventricular septum

A congenital cardiovascular malformation in which both great arteries arise entirely or predominantly from the morphologically left ventricle

A congenital cardiovascular malformation in which a single arterial trunk arises from the heart, giving origin sequentially to the coronary arteries, one or more pulmonary arteries, and the systemic arterial circulation

A congenital cardiovascular malformation in which a common arterial trunk is associated with an unobstructed aortic arch

A congenital cardiovascular malformation in which a common arterial trunk is associated with an unobstructed aortic arch and both pulmonary arteries arise from the common arterial trunk. This category includes Collett-Edwards truncus arteriosus types I, II, III and Van Praagh truncus arteriosus types 1, 2

A congenital cardiovascular malformation in which a common Truncus arteriosus with discontinuous pulmonary arteries; arterial trunk is associated with an unobstructed aortic arch common arterial trunk with discontinuous pulmonary arteries;

and one pulmonary artery does not arise from the common Van Pragh truncus arteriosus type 3 arterial trunk.

It does not include tetralogy of Fallot with pulmonary atresia and absence of the intrapericardial pulmonary arteries (Collett and Edwards truncus arteriosus type IV).

A congenital cardiovascular malformation in which a common arterial trunk is associated with aortic arch obstruction

A congenital cardiovascular malformation in which a common arterial trunk is associated with an interrupted aortic arch

A congenital cardiovascular malformation in which a common arterial trunk is associated with aortic coarctation

A congenital cardiovascular malformation in which there is backward flow through the truncal valve

A congenital cardiovascular malformation in which there is obstruction to flow through the truncal valve due to narrowing or stricture

A congenital cardiovascular malformation where the truncal valve leaflets are markedly thickened with restricted mobility, characterised by the presence of myxomatous tissue

04.00.07 A congenital cardiovascular malformation in which there is an abnormality of a mediastinal vein including but not limited to: pulmonary veins, caval veins, coronary sinus, hepatic veins connecting to the heart, brachiocephalic veins, azygos veins, and/or levo-atrial cardinal veins

Truncus arteriosus; truncus arteriosus communis; persistent

truncus arteriosus; common truncus arteriosus; common

Truncus arteriosus with aortic dominance; truncus arteriosus

with no aortic obstruction

runcus arteriosus with aortic dominance and both pulmonary arteries arising from the trunk

Truncus arteriosus with pulmonary dominance; common arterial trunk with aortic arch obstruction; truncus arteriosus with aortic arch obstruction; Van Praagh truncus arteriosus type 4

Truncus arteriosus with interrupted aortic arch; truncus arteriosus with pulmonary dominance and interrupted aortic arch; common arterial trunk with interrupted aortic arch

Truncus arteriosus with coarctation of aorta; truncus arteriosus with pulmonary dominance and aortic coarctation; common arterial trunk with coarctation of aorta; truncus arteriosus with pulmonary dominance and coarctation of aorta

Congenital truncal valvar incompetence; congenital truncal valvar insufficiency; congenital truncal valve regurgitation Congenital truncal valve stenosis

Congenital truncal valve dysplasia; congenital dysplastic truncal valve; truncal valvar dysplasia 
ICD-11 congenital cardiology terms with

International Paediatric and Congenital

Cardiac Code (IPCCC) numbers, definitions

and synonyms

Congenital anomaly of mediastinal systemic vein $\quad 04.05 .00$ A congenital cardiovascular malformation in which there is an abnormality of a mediastinal systemic vein including but not limited to: caval veins, coronary sinus, hepatic veins connecting to the heart, brachiocephalic veins or azygos veins

Congenital anomaly of superior caval vein

Absent right superior caval vein

Left superior caval vein

Left superior caval vein to coronary sinus

04.01 .01

Left superior caval vein to left-sided atrium

04.01 .02

Congenital anomaly of inferior caval vein

04.03 .08

Interrupted inferior caval vein with absent suprarenal segment and azygos continuation

Congenital anomaly of coronary sinus

Unroofed coronary sinus
A congenital cardiovascular malformation in which there is an abnormality of the superior caval vein (superior vena cava)

A congenital cardiovascular malformation in which there is no right superior caval vein (right superior vena cava) in the setting of the usual atrial arrangement (atrial situs solitus)

A congenital cardiovascular malformation in which there is a left Left superior vena cava; bilateral superior caval vein; bilateral superior caval vein (superior vena cava).

Unless the code for absent right superior caval vein is used, this term assumes that a right superior caval vein is present and, therefore, ther are bilateral superior caval veins with or without a bridging vein

A congenital cardiovascular malformation in which there is a left superior caval vein (superior vena cava) entering a left-sided coronary sinus.

Unless the code for absent right superior caval vein is used, this term assumes that a right superior caval vein is present and, therefore, ther are bilateral superior caval veins with or without a bridging vein

A congenital cardiovascular malformation in which there is a left Left superior caval vein to left-sided atrium; left superior caval superior caval vein (superior vena cava) connecting directly to the left-sided atrium.

Unless the code for absent right superior caval vein is used, this term assumes that a right superior caval vein is present and, therefore, there are bilateral superior caval veins with or without a bridging vein. This term should not be used in the presence of situs inversus of the atria where this is the expected arrangement

A congenital cardiovascular malformation in which there is an abnormality of the inferior caval vein (inferior vena cava)

04.03.10 A congenital cardiovascular malformation in which there is an absence of the renal-to-hepatic segment of the inferior caval vein (inferior vena cava) with connection to a superior caval vein (superior vena cava) through the azygos venous system superior caval veins; persistent left superior vena cava; persistent left superior vena cava

eft superior vena cava to coronary sinus; persistent left superior caval vein to coronary sinus; persistent left superior caval vein to coronary sinus; left superior caval vein persisting to coronary sinus

eft superior caval vein to left-sided
vein persisting to left-sided atrium 政 abnormality of the coronary sinus

04.04.13 A congenital cardiac malformation in which there is direct communication between the left atrium and the coronary sinus.

\section{Congenital anomaly of the inferior vena cava}

Interrupted inferior vena cava with absent suprarenal segment

\section{and azygos continuation}


Coronary sinus orifice atresia or stenosis

04.04 .14

Anomalous hepatic venous connections to the

04.02 .13

heart

Congenital anomaly of pulmonary vein

Anomalous pulmonary venous connection

04.08 .04

04.08 .07

Total anomalous pulmonary venous connection

04.08 .05

Total anomalous pulmonary venous connection of 04.06 .00 the supracardiac type

Total anomalous pulmonary venous connection of 04.08 .10 the cardiac type

Total anomalous pulmonary venous connection of 04.08 .20 the infracardiac type

Total anomalous pulmonary venous connection of 04.08 .30 the mixed type

Partial anomalous pulmonary venous connection $\quad 04.07 .01$

Partial anomalous pulmonary venous connection of 01.01 .16 Scimitar type

Scimitar syndrome

03.02 .23
This term includes partial, and complete unroofing or fenestration of the coronary sinus in the presence or absence of an interatrial communication. If an interatrial communication is present through the coronary sinus orifice then also select the term "interatrial communication through coronary sinus orifice". If a left superior caval vein is present then one should also select the term for "left superior caval vein to left-sided atrium"

A congenital cardiac malformation in which the orifice of the coronary sinus is narrowed or absent

A congenital cardiovascular malformation in which an hepatic vein or hepatic veins do not connect to the inferior caval vein but connect directly to the heart.

NB: this term in ICD-11 sits in developmental anomalies of the liver and child of congenital anomaly of the bepatic vein

A congenital cardiovascular malformation in which there is an Congenital malformation of pulmonary vein abnormality of the pulmonary veins

A congenital cardiovascular malformation in which one or more pulmonary vein does not connect normally to the morphologically left atrium

A congenital cardiovascular malformation in which none of the pulmonary veins connect to the morphologically left atrium

Anomalous pulmonary venous drainage; anomaly of pulmonary veins connection; anomaly of pulmonary venous connection

Totally anomalous pulmonary venous connection; total anomalous pulmonary venous return; total anomalous pulmonary vein connection; total anomalous pulmonary venous drainage

A congenital cardiovascular malformation with total anomalous Total anomalous pulmonary venous connection type 1 pulmonary venous connection to the superior caval vein

(superior vena cava) or one of its venous tributaries

A congenital cardiovascular malformation with total anomalous Total anomalous pulmonary venous connection type 2; total pulmonary venous connection to the right atrium directly or anomalous pulmonary venous connection, intracardiac to the coronary sinus or to both

A congenital cardiovascular malformation with infradiaphragmatic total anomalous pulmonary venous connection

A congenital cardiovascular malformation with total anomalous Total anomalous pulmonary venous connection type 4 pulmonary venous connection at two or more levels (supracardiac, cardiac, or infracardiac)

A congenital cardiovascular malformation in which one or more (but not all) of the pulmonary veins connect anomalously to the right atrium or to one or more of its venous tributaries an the remaining pulmonary veins connect to the left atrium

A congenital cardiovascular malformation with partial anomalous Partial anomalous pulmonary venous return of Scimitar type pulmonary venous connection in which some of the pulmonary veins (usually the right pulmonary veins) connect anomalously to the inferior caval vein (inferior vena cava) or to the right atrium at the insertion of the inferior vena cava

A congenital cardiopulmonary malformation with "partial anomalous pulmonary venous connection of Scimitar type" and one or more of the following: hypoplasia of the right lung with bronchial anomalies, dextrocardia, hypoplasia of the right pulmonary artery, lobar lung sequestration, and anomalous systemic arterial supply to the lower lobe of the right lung directly from the aorta or its main branches
Partially anomalous pulmonary venous connection; partia anomalous pulmonary venous return; partial anomalous pulmonary venous drainage

Pulmonary venolobar syndrome 
ICD-11 congenital cardiology terms with

International Paediatric and Congenital

Cardiac Code (IPCCC) numbers, definitions

and synonyms

Obstructed anomalous pulmonary venous pathway 04.08 .06 or connection

Congenital pulmonary venous stenosis $\quad 04.08 .31$ or hypoplasia

Congenital atresia of pulmonary vein

04.08 .02

Congenital anomaly of an atrium or atrial septum 05.00 .02

Congenital anomaly of atrial septum

05.07 .01

Restrictive interatrial communication or intact

atrial septum when an interatrial shunt is

physiologically necessary

Aneurysm of atrial septum

05.03 .03

Interatrial communication

05.04 .01

Patent oval foramen

05.03 .01

Atrial septal defect within oval fossa
A congenital cardiovascular malformation in which the pathway of Obstructed anomalous pulmonary venous return; obstructed one or more anomalous pulmonary veins is blocked or impeded anomalous pulmonary venous connection

A congenital cardiovascular malformation with a pathologic Congenital pulmonary vein stenosis and/or hypoplasia narrowing of one or more pulmonary veins including diffuse

hypoplasia, long-segment focal/tubular stenosis and/or discrete stenosis

A congenital cardiovascular malformation with atresia of one or Congenital pulmonary vein atresia more pulmonary veins

A congenital cardiovascular malformation in which there is an abnormality of an atrium and/or atrial septum

A congenital cardiac malformation in which there is an abnormality of the atrial septum

A cardiac finding in which there is either absence of an interatrial Restrictive interatrial communication in the presence of an communication or the communication is smaller than is required to permit physiologically adequate interatrial flow. This is always an abnormal finding prenatally but is a postnatal finding that should only be coded when present prenatally or when associated with other cardiac abnormalities that result in physiological disturbances secondary to the absence of a larger interatrial communication - such as mitral or tricuspid valve atresia

A congenital cardiac finding in which the septum primum is abnormally large (redundant) and results in aneurysmal protrusion into one or both atria

A congenital cardiac malformation in which there is a hole or pathway between the atrial chambers.

Although loosely often termed an "atrial septal defect", not all interatrial communications have a defect in the true atrial septum, and so "atrial septal defect" in this context is not a true synonym

A congenital cardiovascular finding in which there is a small interatrial communication (or potential communication) confined to the region of the oval fossa (fossa ovalis)

characterized by no deficiency of the primary atrial septum (septum primum) and a normal limbus with no deficiency of the septum secundum (superior interatrial fold)

A congenital cardiac malformation in which there is an

interatrial communication confined to the region of the oval fossa (fossa ovalis), most commonly due to a deficiency of the primary atrial septum (septum primum) but deficiency of the septum secundum (superior interatrial fold) may also contribute
Congenital malformation of atrial septum

obligatory interatrial shunt

Atrial septal defect

Patent foramen ovale; open foramen ovale; open oval foramen; persistent foramen ovale

Secundum atrial septal defect; ostium secundum atrial septa defect 
Common atrium with separate atrioventricular junctions

Interatrial communication through coronary sinus orifice

Congenital anomaly of right atrium

05.01 .13

Divided right atrium

Left-sided juxtaposition of the atrial appendages

05.01 .06

Congenital giant right atrium

Congenital anomaly of left atrium

Divided left atrium
A congenital cardiovascular malformation in which there is a Sinus venosus communication; sinus venosus atrial septal defect caval vein (vena cava) and/or pulmonary vein (or veins) that overrides the atrial septum or the septum secundum (superior interatrial fold) producing an interatrial or anomalous veno-atrial communication.

Although the term sinus venosus atrial septal defect is commonly used. the lesion is more properly termed a sinus venosus communication because, while it functions as an interatrial communication, this lesion is not a defect of the atrial septum

A congenital cardiovascular malformation in which there is complete or near-complete absence of the interatrial septum

A congenital cardiovascular malformation in which there is a communication between the left atrium and the coronary sinus allowing interatrial communication through the coronary sinus ostium.

"Interatrial communication through coronary sinus orifice" may or may not be associated with a persistent left superior caval vein (superior vena cava). This occurs in the absence of the coronary sinus (total unroofing of the coronary sinus) or partial unroofing of the coronary sinus

A congenital cardiac malformation in which there is an abnormality of the right atrium

A congenital cardiac malformation in which the right atrium is Cor triatriatum dexter; cor triatriatum dextrum; right cor partially divided by a large or obstructive Eustachian valve triatriatum

A congenital cardiac malformation in which the right atrial appendage extends from the right atrium, behind the great arteries, to lie adjacent to the left atrial appendage.

This is frequently associated with horizontal orientation of the atrial septum, hypoplasia or atresia of the tricuspid valve, bypoplasia of the right ventricle, transposition of the great arteries or double outlet right ventricle, and subpulmonary or subaortic stenosis

A congenital cardiac malformation in which the right atrium is Congenital right atrial aneurysm severely dilated. This is an isolated finding not secondary to abnormalities of the tricuspid valve or right ventricle

A congenital cardiac malformation in which there is an abnormality of the left atrium

05.02.01 A congenital cardiac malformation in which there is a partition Cor triatriatum sinister; cor triatriatum sinistrum; left cor that divides the left atrium into a posterior chamber that receives some or all of the pulmonary veins and an anterior chamber that communicates with the left atrial appendage and atrioventricular junction (usually the mitral valve). In differentiating cor triatriatum from supravalvar mitral ring, in cor triatriatum the posterior compartment contains the pulmonary veins while the anterior contains the left atrial appendage and atrioventricular junction (usually the mitral valve); in supravalvar mitral ring, the anterior compartment contains only the mitral valve orifice 
ICD-11 congenital cardiology terms with

International Paediatric and Congenital

Cardiac Code (IPCCC) numbers, definitions

Right-sided juxtaposition of the atrial appendages 05.02 .04

Congenital anomaly of an atrioventricular valve or 06.00 .15 atrioventricular septum

Congenital anomaly of tricuspid valve

Congenital tricuspid regurgitation

Congenital tricuspid valvar stenosis

Tricuspid annular hypoplasia

Dysplasia of tricuspid valve

Straddling tricuspid valve

06.01 .09

Overriding tricuspid valve

06.01 .05
A congenital cardiac malformation in which the left atrial appendage extends from the left atrium, behind the great arteries, to lie adjacent to the right atrial appendage.

This is commonly associated with hypoplasia or atresia of the mitral valve, hypoplasia of the left ventricle, normal (i.e. subpulmonary only) conus and may be seen with atrioventricular septal defect (common atrioventricular canal) or subaortic or aortic stenosis or atresia

A congenital cardiac malformation in which there is an abnormality of an atrioventricular valve or atrioventricular septum

A congenital cardiac malformation in which there is an abnormality of the tricuspid valve.

Tricuspid atresia is listed in the functionally univentricular heart section. Examples of terms included here are: True cleft of tricuspid valve leaflet; congenital anomaly of tricuspid subvalvar apparatus: absent tricuspid valve leaflet; unguarded tricuspid orifice; and congenital deformity of tricuspid valve

A congenital cardiac finding in which there is backward flow through the tricuspid valve

Congenital tricuspid insufficiency; congenital tricuspid incompetence; congenital tricuspid valve regurgitation;

congenital tricuspid valve insufficiency; congenital tricuspid valve incompetence

A congenital cardiovascular malformation of the tricuspid valve Congenital tricuspid valve stenosis; congenital stenosis of in which there is narrowing or stricture (obstruction to flow) tricuspid valve; congenital tricuspid stenosis

A congenital cardiac malformation of the tricuspid valve in Hypoplasia of tricuspid valvar annulus; hypoplasia of the which there is annular hypoplasia (incomplete development tricuspid annulus or underdevelopment so that it is abnormally small [below the lower limit of normal adjusted for body size]).

Hypoplasia may or may not be associated with stenosis

A congenital cardiac malformation of the tricuspid valve, commonly consisting of leaflet thickening and restricted mobility, with normally hinged leaflets.

This diagnosis is not used for patients with Ebstein malformation of tricuspid valve, which is characterised by abnormally binged tricuspid valve

A congenital cardiac malformation in which the tricuspid subvalvar apparatus has attachments within both ventricles. This may or may not be associated with valvar overriding

A congenital cardiac malformation in which the tricuspid valve annulus lies in part above both the right and left ventricles

\section{Tricuspid valvar dysplasia; dysplastic tricuspid valve}


Ebstein malformation of tricuspid valve

06.01 .34

Congenital anomaly of mitral valve

Congenital mitral regurgitation

Congenital mitral valvar stenosis

Mitral annular hypoplasia

Straddling mitral valve

06.02 .11

06.02 .04
A congenital cardiac malformation of the tricuspid valve and right ventricle that is characterised by downward (apical) displacement of the functional annulus, usually involving the septal and inferior (posterior) leaflets.

Ebstein anomaly is a malformation of the tricuspid valve and right ventricle that is characterised by a spectrum of several features: (1) incomplete delamination of septal and inferior (posterior) tricuspid valve leaflets from the myocardium of the right ventricle; (2) downward (apical) displacement of the functional annulus; (3) dilation of the "atrialized" portion of the right ventricle with variable degrees of bypertrophy and thinning of the wall; (4) redundancy, fenestrations, and tethering of the anterosuperior leaflet and (5) dilation of the right atrioventricular junction (the true tricuspid annulus). These anatomical and functional abnormalities cause tricuspid regurgitation (and rarely tricuspid stenosis) that results in right atrial and right ventricular dilation and atrial and ventricular arrhythmias. Associated cardiac anomalies include an interatrial communication, the presence of accessory conduction pathways and dilation of the right atrium and right ventricle.

Varying degrees of right ventricular outflow tract obstruction may be present, including pulmonary atresia. Some patients with discordant atrioventricular and ventriculo-arterial connections [congenitally corrected transposition] have an Ebstein-like deformity of the leftsided morphologically tricuspid valve

A congenital cardiac malformation in which there is an abnormality of the mitral valve.

Mitral atresia is listed in the functionally univentricular heart

section. Examples of terms included here are: congenital unguarded mitral orifice; accessory tissue on mitral valve leaflet; and double orifice of mitral valve leaflet

A congenital cardiac finding in which there is backward flow through the mitral valve

A congenital cardiac malformation of the mitral valve in which there is narrowing or stricture of the valvar orifice (obstruction to flow)

A congenital cardiac malformation of the mitral valve in which there is annular hypoplasia (incomplete development or underdevelopment so that it is abnormally small [below the lower limit of normal adjusted for body size]). Hypoplasia may or may not be associated with stenosis

A congenital cardiac malformation in which the mitral subvalvar apparatus has attachments onto the crest of the ventricular septum or within both ventricles.

This may or may not be associated with valvar overriding
Ebstein syndrome; Ebstein anomaly; Ebstein disease; Ebstein anomaly of tricuspid valve; Congenital Ebstein deformity of tricuspid valve

\section{Congenital mitral valve anomaly}

Congenital mitral insufficiency; congenital mitral incompetence; congenital insufficiency of mitral valve; congenital mitral valve regurgitation; congenital mitral valve insufficiency; congenital mitral valve incompetence

disease; congenital mitral stenosis; congenital stenosis of mitral valve

Hypoplasia of mitral valvar annulus; hypoplasia of the mitral annulus 
ICD-11 congenital cardiology terms with

International Paediatric and Congenital

Cardiac Code (IPCCC) numbers, definitions

and synonyms

Overriding mitral valve

Dysplasia of mitral valve

Supravalvar or intravalvar mitral ring

05.02 .02

Congenital mitral valvar prolapse

06.02 .72

True cleft of anterior mitral leaflet

06.02 .36

Congenital anomaly of mitral subvalvar apparatus $\quad 06.02 .21$

Congenital mitral subvalvar stenosis

06.02 .22

Parachute malformation of mitral valve

06.02 .56

Congenital anomaly of left-sided atrioventricular $\quad 06.04 .11$ valve in double inlet ventricle
A congenital cardiac malformation in which the mitral valve annulus lies in part above both the right and left ventricles

A congenital cardiac malformation that includes any structural Mitral valve dysplasia; dysplastic mitral valve abnormality of the mitral valvar leaflet(s), commonly consisting of leaflet thickening and restricted mobility

A congenital cardiac malformation in which a ridge of tissue is Supravalvar mitral membrane; Membranous supravalvular mitral attached or integral to the atrial side of the mitral valvar stenosis; Congenital supravalvar mitral ring; supravalvar mitral leaflet(s).

The intravalvar variant is distinguished from the supravalvar variant because the intravalvar ring is within the funnel of the mitral valvar leaflets. Both the intravalvar variant and the supravalvar variant are differentiated from cor triatriatum because in cor triatriatum the posterior compartment contains the pulmonar veins while the anterior contains the left atrial appendage (and the atrioventricular valve [usually mitral valve] in the absence of left atrioventricular valvar atresia); in supravalvar or intravalvar mitral ring, the anterior compartment contains only the mitral valve orifice and not the left atrial appendage

A congenital cardiac malformation of the mitral valve in which one or both leaflets move to the atrial side of the plane of the annulus in systole

A congenital cardiac malformation of the mitral valve in which Isolated cleft of the anterior mitral leaflet (without common the anterior leaflet is divided into two parts.

This term should not be used for the so-called "mitral cleft" of a common atrioventricular valve, as this is the zone of apposition between the superio and inferior bridging leaflets, and not a divided valve leaflet

A congenital cardiac malformation in which the mitral chords, chordal attachments, or papillary muscles are abnormal

A congenital cardiac malformation in which there is stenosis (narrowing or stricture of a duct or a canal) of the subvalvar components (chordae tendineae and/or papillary muscles) of the mitral valve. This diagnosis includes mitral stenosis associated with parachute mitral valve, mitral arcade, and hammock mitral valve

A congenital cardiac malformation in which the chords of the mitral Parachute mitral valve valve attach to a single or to closely adjacent papillary muscles

A congenital cardiac malformation in association with double inlet ventricle (excluding common atrioventricular valve) in which the atrioventricular valve morphology cannot be determined. This term should be used for the left-sided atrioventricular valve in those hearts atrioventricular junction)

Subvalvar mitral stenosis, congenital mitral stenosis stenosis 
Congenital anomaly of right-sided atrioventricular 06.03 .11 valve in double inlet ventricle

Common atrioventricular junction

Atrioventricular septal defect

06.06 .00

Atrioventricular septal defect with balanced ventricles

Atrioventricular septal defect with ventricula imbalance

Atrioventricular septal defect with ventricula imbalance with dominant right ventricle,

hypoplastic left ventricle

Atrioventricular septal defect with ventricula

imbalance with dominant left ventricle

hypoplastic right ventricle

Atrioventricular septal defect with communication 06.06.01 at the atrial level only

06.07 .27

06.07 .26

06.07 .05
A congenital cardiac malformation in association with double inlet ventricle (excluding common atrioventricular valve) in which the atrioventricular valve morphology cannot be determined. This term should be used for the right-sided atrioventricular valve in those hearts

A congenital cardiac malformation where both atria connect to a Common atrioventricular canal common atrioventricular valve which characteristically has four or five leaflets including superior and inferior bridging leaflets with a single annulus. The common valve may have one or two major orifices depending on the absence or presence of fusion of the bridging leaflets to each other or the septal crest.

The left ventricular zone of apposition between the superior and inferior bridging leaflets is commonly referred to as a "cleft"

A congenital cardiac malformation with a common atrioventricular Atrioventricular canal defect junction and an atrioventricular septal defect

A congenital cardiac malformation that is a variant of atrioventricular septal defect (atrioventricular canal defect)

with ventricles that are equal or nearly equal in size

A congenital cardiac malformation that is a variant of

Balanced atrioventricular canal atrioventricular septal defect (atrioventricular canal defect) with one ventricle significantly larger than the other.

Unbalanced ventricular size and unbalanced relation of the common atrioventricular valve to the ventricles are to be distinguished by coding unbalanced ventricular size as "Atrioventricular septal defect with ventricular imbalance" and the unbalanced relation of the common atrioventricular valve to the ventricles should also be coded as "Common atrioventricular valve with unbalanced commitment of valve to ventricles"

A congenital cardiac malformation that is a variant of

Right dominant atrioventricular septal defect/atrioventricular atrioventricular septal defect (atrioventricular canal defect) canal; complete atrioventricular septal defect with left with the right ventricle significantly larger than the left ventricular hypoplasia

A congenital cardiac malform atrioventricular septal defect (atrioventricular canal defect) with the left ventricle significantly larger than the right

A congenital cardiac malformation that is a variant of an atrioventricular septal defect (atrioventricular canal defect) with an interatrial communication just above the atrioventricular valve, no interventricular communication just below the atrioventricular valve, separate right and left atrioventricular valvar orifices, and varying degrees of malformation of the left-sided component of the common atrioventricular valve. The bridging leaflets of the common atrioventricular valve are bound down to the crest of the scooped-out ventricular septum so that the potential for shunting through the atrioventricular septal defect is possible only at the atrial level and not at the ventricular level

Left dominant atrioventricular septal defect/atrioventricular canal; complete atrioventricular septal defect with right ventricular hypoplasia

Primum atrial septal defect; partial atrioventricular canal defect; incomplete atrioventricular septal defect with isolated atria component; incomplete atrioventricular canal defect with isolated atrial component; ostium primum atrial septal defect; atrioventricular defect with atrial shunting only; partial atrioventricular canal defect with isolated atrial component; partial atrioventricular septal defect: ostium primum type; partial atrioventricular septal defect 
ICD-11 congenital cardiology terms with

International Paediatric and Congenital

Cardiac Code (IPCCC) numbers, definitions and synonyms

\section{IPCCC code Definition with commentar}

Atrioventricular septal defect with communication 06.06.08 at the ventricular level only

Atrioventricular septal defect with communication 06.06 .10 at atrial level and restrictive communication at ventricular level

Atrioventricular septal defect with communication 06.06.09 at atrial level and unrestrictive communication at ventricular level at the atrial level ventricular level" should be used
A congenital cardiac malformation that is a variant of an atrioventricular septal defect (atrioventricular canal defect) with an interventricular communication just below the atrioventricular valve, no interatrial communication just above the atrioventricular valve, separate right and left atrioventricular valvar orifices, and varying degrees of malformation of the left-sided component of the common atrioventricular valve. The bridging leaflets of the common atrioventricular valve are bound to the atrial septum so that the potential for shunting through the atrioventricular septal defect is possible only at the ventricular level and not

A congenital cardiac malformation that is a variant of an atrioventricular septal defect (atrioventricular canal defect) with an interatrial communication immediately above the atrioventricular valve, and a restrictive interventricular communication immediately below the atrioventricular valve. This term is used to identify bearts with a restrictive interventricular communication. If there is a single atrioventricular valvar orifice this term should still be used. If the ventricular component of the atrioventricular septal defect is unrestrictive (no interventricular pressure gradient), this malformation should not be coded as "Atrioventricular septal defect (atrioventricular canal defect) with communication at atrial level and restrictive communication at ventricular level" and instead the term "Atrioventricular septal defect (atrioventricular canal defect) with communication at atrial level and unrestrictive communication at

A congenital cardiac malformation that is a variant of an atrioventricular septal defect (atrioventricular canal defect) with an interatrial communication just above the atrioventricular valve, an interventricular communication just below the atrioventricular valve, and varying degrees of malformation of the left ventricular component of the common atrioventricular valve. There is unrestrictive interventricular communication (no interventricular pressure gradient) and the bridging leaflets usually float to varying extent within the atrioventricular septal defect

A congenital cardiac malformation with both an atrioventricular Atrioventricular canal and tetralogy of Fallot septal defect (atrioventricular canal defect) and tetralogy of Fallot.
Synonyms

Atrioventricular canal defect with isolated ventricular communication; atrioventricular canal defect with isolated ventricular component; atrioventricular septal defect with isolated ventricular component

Intermediate atrioventricular septal defect with atrial and ventricular components and separate atrioventricular valvar orifices; atrioventricular canal defect associated with a restrictive ventricular septal defect; atrioventricular septal defect with atrial shunting and restrictive ventricular shunting; intermediate atrioventricular canal defect with atrial and ventricular components and separate atrioventricular valves; transitional atrioventricular canal defect with atrial an ventricular components and separate atrioventricular valves; transitional atrioventricular septal defect with atrial and ventricular components and separate atrioventricular valves

Complete atrioventricular septal defect; complete atrioventricular canal defect; complete atrioventricular canal with atrial and ventricular components 
Common atrium with common atrioventricular junction

Common atrioventricular valvar regurgitation

Atypical common atrioventricular valve

Common atrioventricular valve with unbalanced $\quad 06.07 .36$ commitment of valve to ventricles

Common atrioventricular valve with unbalanced

06.07 .37

commitment of valve to right ventricle

Common atrioventricular valve with unbalanced $\quad 06.07 .38$ commitment of valve to left ventricle

Atypical right ventricular component of common 06.05 .71 atrioventricular valve

Atypical left ventricular component of common atrioventricular valve

Double orifice of left ventricular component of common atrioventricular valve

Deficient mural leaflet of left ventricular component of common atrioventricular valve

06.05 .25

06.05 .98
Tetralogy of Fallot with atrioventricular septal defect (common atrioventricular canal) is always the complete form (unrestrictive interventricular component) and usually has four leaflets of the common atrioventricular valve (undivided superior leaflet) and very few or no attachments of that leaflet to the crest of the ventricular septum

A congenital cardiac malformation in which there is complete absence of the interatrial septum in the setting of a common atrioventricular junction (common atrioventricular canal)

06.05.60 A congenital cardiac finding where there is backward flow through the common atrioventricular valve

A congenital cardiac malformation in which the common atrioventricular valve does not have the usual morphology of four to five non-dysplastic leaflets that relate relatively equally to both ventricles

A congenital cardiac malformation in which the common atrioventricular valve is primarily related to one ventricle, usually but not always associated with hypoplasia of the other ventricle.

Unbalanced ventricular size and unbalanced relation of the common atrioventricular valve to the ventricles are to be distinguished by coding unbalanced ventricular size as "Atrioventricular septal defect with ventricular imbalance" and the unbalanced relation of the common atrioventricular valve to the ventricles should also be coded as "Common atrioventricular valve with unbalanced commitment of value to ventricles"

A congenital cardiac malformation in which the common atrioventricular valve is primarily related to the right ventricle, usually but not always associated with hypoplasia of the left ventricle

A congenital cardiac malformation in which the common atrioventricular valve is primarily related to the left ventricle, usually but not always associated with hypoplasia of the right ventricle

A congenital cardiac malformation in which the right ventricular component of the common atrioventricular valve does not have the usual structure (independently supported and well developed right ventricular components of the common atrioventricular valve)

A congenital cardiac malformation in which the left ventricular component of the common atrioventricular valve does not have the usual structure (independently supported and well developed left ventricular components of the common atrioventricular valve)

A congenital cardiac malformation in which the left ventricular component of the common atrioventricular valve has two orifices

A congenital cardiac malformation in which the left ventricular component of the common atrioventricular valve is composed primarily of the bridging leaflets due to

Deficient lateral leaflet of left ventricular component of common atrioventricular valve
Unbalanced relation of the common atrioventricular valve to the ventricles 
ICD-11 congenital cardiology terms with

International Paediatric and Congenital

Cardiac Code (IPCCC) numbers, definitions

and synonyms

Common atrioventricular junction with spontaneous fibrous closure of atrioventricular septal defect

Communication between left ventricle and right

07.14 .02 atrium

Congenital anomaly of a ventricle or the ventricular septum

Congenital right ventricular anomaly

Right ventricular hypoplasia

07.02 .00
A congenital cardiac malformation in which there is a common atrioventricular junction without any communication at the level of the atrioventricular septum.

This code could be used when there is a trifoliate left atrioventricular valve, commonly known as cleft mitral valve secondary to atrioventricular septal defect, and also has evidence of spontaneous obliteration of the atrioventricular septal defect by valve or subvalvar tissue. Additional defects in the atrial or ventricular septa that do not involve the atrioventricular septum are not excluded by this term and should be coded separately

A deficiency of the atrioventricular component of the membranous septum permitting shunting of blood from the left ventricle to the right atrium.

This is an isolated absence of the atrioventricular septum without other components that may be commonly seen in association with spectrum of disorders associated with atrioventricular septal defects such as a common atrioventricular valve or interatrial or interventricular communications. The Gerbode defect may be confused with ventricular septal defects where a deficiency of tricuspid valvar tissue permits ventriculo-atrial shunting subsequent to initial interventricular shunting (left ventricle to right ventricle to right atrium)

Atrioventricular septal defect with intact septal structures; common atrioventricular junction without an atrioventricula septal defect

Gerbode defect

mital cardiac malformation in which there is an

abnormality of a ventricle and/or the ventricular septum.

The ventricles include the ventricular inlet, ventricular body and ventricular outflow tract

A congenital cardiac malformation in which there is an abnormality of the right ventricle

A congenital cardiac malformation in which the right ventricle is Hypoplastic right ventricle abnormally small (below the lower limit of normal adjusted for body size).

This morphological abnormality usually is an integral part of other congenital cardiovascular anomalies and, therefore, frequently does not need to be coded separately. It should, however, be coded as secondary to an accompanying congenital cardiovascular anomaly if the right ventricular hypoplasia is not considered an integral and understood part of the primary congenital cardiovascular diagnosis. It would rarely be coded as a primary andlor isolated diagnosis

07.05.20 A congenital cardiac condition in which the flow through the right ventricular outflow tract (proximal to the valve[s] guarding the outflow from the right ventricle) is blocked or impeded 
Double-chambered right ventricle

Right ventricular myocardial sinusoids

Parchment right ventricle

Tetralogy of Fallot

Tetralogy of Fallot with absent pulmonary valve 09.05 .25 syndrome
A congenital cardiac malformation in which the right ventricle is Double-chambered right ventricle; anomalous right ventricular divided into two chambers, one inferior including the inlet muscle bundle

and trabecular portions of the right ventricle and one superior

including the trabecular portion and infundibulum.

Double chamber right ventricle is often associated with one or several closing

ventricular septal defects. In some cases, the ventricular septal defect is

already closed. Double chamber right ventricle is differentiated from the

rare isolated infundibular stenosis that develops more superiorly

A congenital cardiac malformation in which there are deep,

endothelial lined, blind-ended intramyocardial tunnels

communicating with the right ventricular chamber. These

occur in the setting of pulmonary atresia or severe right

ventricular outflow tract obstruction, intact ventricular

septum and a patent tricuspid valve.

These right ventricular sinusoids do not communicate with the

coronary arteries. Where connections from the right ventricle to the coronary arteries exist, one should code for "Congenital coronary artery-to-right ventricular fistula"

A congenital cardiac malformation in which there is almost complete absence of right ventricular myocardium and preserved septal and left ventricular myocardium.

Parchment right ventricle can occur in association with a normal tricuspid valve (Ubl anomaly) or with an abnormal tricuspid valve, such as Ebstein Anomaly. This should be distinguished from arrhythmogenic right ventricular cardiomyopathy by the presence of fatty infltration in the latter

A group of congenital cardiac malformations with biventricular Ventricular septal defect with obstructed right ventricular atrioventricular alignments or connections characterised by anterosuperior deviation of the conal or outlet septum or its fibrous remnant, narrowing, or atresia of the pulmonary outflow, a ventricular septal defect of the malalignment type, and biventricular origin of the aorta. Tetralogy of Fallot will always have a ventricular septal defect, narrowing or atresia of the pulmonary outflow, aortic override, and most often right ventricular hypertrophy

A congenital cardiovascular malformation that is a variant of tetralogy of Fallot in which the ventriculo-arterial junction of the right ventricle with the pulmonary trunk features an atypical valve with absent or rudimentary leaflets (cusps) that do not coapt. In its usual form there is dilatation of the pulmonary trunk and central right and left pulmonary arteries, which when extreme, is associated with abnormal arborisation of lobar and segmental pulmonary artery branches and with compression of the trachea and main stem bronchi, often with tracheobronchomalacia. The physiologic consequence is usually a combination of variable degrees of both stenosis and regurgitation of the pulmonary valve
Uhl's anomaly; right ventricular wall paper thin

outflow; tetrad of Fallot; Fallot tetralogy; Fallot tetrad; Fallot disease; Fallot complex; Fallot's tetralogy; subpulmonic ventricular hypertrophy; ventricular septal defect with

pulmonary stenosis or atresia, dextraposition of aorta, and stenosis, ventricular septal defect, overriding aorta, and right

hypertrophy of right ventricle 
ICD-11 congenital cardiology terms with

International Paediatric and Congenital

Cardiac Code (IPCCC) numbers, definitions and synonyms

Tetralogy of Fallot with pulmonary atresia

01.01 .26

Tetralogy of Fallot with pulmonary atresia and systemic-to-pulmonary collateral artery

01.01 .57

Congenital left ventricular anomaly

07.06 .07

Left ventricular hypoplasia

07.07 .00

Congenital left ventricular aneurysm or diverticulum

Congenital left ventricular outflow tract obstruction

Left ventricular outflow tract obstruction due to 07.09 .08 atrioventricular valve

Left heart obstruction at multiple sites
A congenital cardiovascular malformation that is a variant of tetralogy of Fallot in which there is no direct

communication between the right ventricle and the pulmonary arterial tree

A congenital cardiovascular malformation that is a variant of tetralogy of Fallot in which there is no direct

communication between the right ventricle and the pulmonary arterial tree and there are collateral blood vessels between the systemic and pulmonary arteries

A congenital cardiac malformation in which there is an abnormality of the left ventricle

A congenital cardiac malformation in which the left ventricle is Hypoplastic left ventricle abnormally small (below the lower limit of normal adjusted for body size)

This morphological abnormality usually is an integral part of other congenital cardiovascular anomalies and does not need to be coded separately if this is the case. It should be coded as secondary to an accompanying congenital cardiovascular anomaly if the left ventricular hypoplasia is not considered an integral and understood part of the primary congenital cardiovascular diagnosis such as bypoplastic left heart syndrome

07.06.19 A congenital cardiac malformation in which there is an outpouching of the left ventricular wall

07.09.28 A congenital cardiac condition in which the flow through the left ventricular outflow tract (proximal to the valve[s] guarding the outflow from the left ventricle) is blocked or impeded. This code should not be used for obstruction immediately under the arterial valve such as subartic stenosis due to fibromuscular shelf or tunnel

A congenital cardiac malformation in which the flow through the left ventricular outflow tract is blocked or impeded due to abnormal position or attachments of tricuspid, mitral, or common atrioventricular valvar tissue

A congenital cardiovascular malformation in which more than one of the following lesions are present: (1) supravalvar or intravalvar mitral ring; (2) mitral subvalvar stenosis; (3) a parachute deformity of the mitral valve; (4) subaortic

stenosis; (5) valvar aortic stenosis; and (6) aortic coarctation. Shone's syndrome consists of multilevel hypoplasia or obstruction of the left heart. The syndrome is based on the original report from Shone that was based on analysis of eight autopsied cases and described the tendency of these four obstructive, or potentially obstructive, conditions to coexist. Only two of the eight cases exhibited all four conditions. with the other cases exbibiting only two or three of the anomalies
Pulmonary atresia with ventricular septal defect, Fallot type

Pulmonary atresia with ventricular septal defect and systemic-topulmonary collateral arteries [Fallot type]; pulmonary atresia, ventricular septal defect and MAPCA 
Left ventricular myocardial sinusoids

Congenital anomaly of ventricular septum

07.20 .04

Restrictive interventricular communication when an interventricular shunt is physiologically necessary

Ventricular septal defect
A congenital cardiac malformation in which there are deep, endothelial lined, blind-ended intramyocardial tunnels communicating with the left ventricular chamber. These occur in the setting of aortic atresia or severe left ventricular outflow tract obstruction, intact ventricular septum and a patent mitral valve.

These left ventricular sinusoids do not communicate with the coronary arteries. Where connections from the left ventricle to the coronary arteries exist, one should code for "Congenital coronary artery-to-left ventricular fistula". This code should not be used for left ventricular non-compaction

A congenital cardiac malformation in which there is an abnormality of the interventricular septum

A congenital cardiac finding in which there is an interventricular communication that is smaller than is required to permit physiologically adequate interventricular flow.

This should be coded only when associated with other cardiac

abnormalities that result in physiological disturbances secondary to the absence of a larger interventricular communication. Clinically

important examples include double outlet right ventricle or tetralogy of Fallot with restrictive ventricular septal defect, double inlet left ventricle, or tricuspid atresia with concordant or discordant great arterial connections and restrictive ventricular septal defect, and others

A congenital cardiac malformation in which there is a hole or pathway between the ventricular chambers.

The definitions offered for a "ventricular septal defect", in its various forms, will be used most frequently in the setting of patients who do not have abnormalities of either the atrioventricular or ventriculoarterial connections. The definitions themselves, however, are equally applicable for the description and categorisation of holes or pathways between the ventricles when the segmental connections between the cardiac components are abnormal. The key to understanding the definitions is to appreciate that the hole or pathway between the ventricles is defined both on the basis of its geographic location within the ventricular septum and its margins as seen from the aspect of the morphologically right ventricle. In this respect, when the ventriculo-arterial connections are concordant or discordant, the roof of the channel, as viewed from the right ventricle, is the muscular outlet septum or its fibrous remnant. When both arterial trunks arise from the morphologically right ventricle, in contrast, the channel between the ventricles provides the outflow from the morphologically left ventricle, and is roofed by the inner heart curvature. In the setting of double outlet right ventricle, therefore, the interventricular communication is not the same geometric locus as the ventricular septal defect, although many still continue to describe the outlet from the left ventricle as the "ventricular septal defect". In univentricular atrioventricular connections with functionally single left ventricle with an outflow chamber, the communication, which used to be
Ventricular septal abnormality; ventricle septa anomaly; ventricle septal heart anomaly; ventricle septum heart anomaly; ventricular septal heart anomaly; ventricular septa anomaly

Interventricular communication; interventricular septal defect; interventricular septum defect; ventricular septum defect 
ICD-11 congenital cardiology terms with

International Paediatric and Congenital

Cardiac Code (IPCCC) numbers, definitions

and synonyms

called a bulboventricular foramen, is both a ventricular septal defect and an interventricular communication. The situation in the setting of double outlet right ventricle, however, shows that the terms cannot always be used as being synonymous. A similar situation pertains in the setting of common arterial trunk, since the bole usually closed by the surgeon to provide septal integrity is the right ventricular margin of the channel between the ventricles, rather than the geometric interventricular communication

Perimembranous central ventricular septal defect $\quad 07.10 .0$

A congenital cardiovascular malformation in which there is a ventricular septal defect that: 1) occupies the space that is usually closed by the interventricular part of the membranous septum, 2) is adjacent to the area of fibrous continuity between the leaflets of an atrioventricular valve and an arterial valve, and 3 ) is located at the centre of the base of the ventricular mass.

Although best used to describe the perimembranous defect that opens centrally at the base of the right ventricle, this term might be used to code perimembranous defects with inlet or outlet extension. It is recommended, however, that the more precise terms be used whenever possible for coding the latter lesions. This code is used by some as synonymous with the perimembranous, conoventricular, Type II, or the paramembranous defects. It should not be used to code an inlet ventricular septal defect, or the so-called atrioventricular canal ventricular septal defect. More specific terms exist for coding these entities. It is used by some to describe an isolated perimembranous ventricular septal defect without extension, although it is unlikely that perimembranous defects exist in the absence of deficiency of their muscular perimeter. The conoventricular ventricular septal defect with malalignment should be coded as an outlet defect, as should the perimembranous defect opening to the outlet of the right ventricle. All perimembranous defects, nonetheless, have part of their margins made up of fibrous continuity either between the leaflets of an atrioventricular and an arterial valve or, in the setting of double outlet right ventricle or overriding of the tricuspid valve, by fibrous continuity between the leaflets of the mitral and tricuspid valves.

Such defects can also extend to become doubly committed and juxtaarterial (conal septal bypoplasia) when there is also fibrous

continuity between the leaflets of the arterial valves or when there is a common arterial valve. Specific codes exist for these variants, which ideally should not be coded using this term

Perimembranous ventricular septal defect; membranous ventricular septal defect; paramembranous ventricular septal defect; type 2 ventricular septal defect; central perimembranous ventricular septal defect 
Inlet ventricular septal defect without a common

07.14 .05 atrioventricular junction

Inlet perimembranous ventricular septal defect without atrioventricular septal malalignment without a common atrioventricular junction

Inlet perimembranous ventricular septal defect with atrioventricular septal malalignment and without a common atrioventricular junction

Inlet muscular ventricular septal defect

Trabecular muscular ventricular septal defect

07.11 .01

Trabecular muscular ventricular septal defect: midseptal

Trabecular muscular ventricular septal defect: apical

07.14 .06

07.11 .03
A congenital cardiac malformation in which there is a ventricular Inlet ventricular septal defect; type 3 ventricular septal defect; septal defect that opens predominantly into the inlet component of the right ventricle in the absence of a common atrioventricular junction

07.10.02 A congenital cardiac malformation in which there is a ventricular Perimembranous inlet ventricular septal defect; perimembranous septal defect that usually, but not always, extends beneath the full annular length of the septal leaflet of the tricuspid valve and to the membranous septum, into the inlet component of the right ventricle in the absence of atrioventricular septal malalignment and of a common atrioventricular junction

A congenital cardiac malformation in which there is an inlet ventricular septal defect with malalignment between the atrial septum and the postero-inferior part of the muscular ventricular septum, in the absence of a common

atrioventricular junction, such that there is overriding with or without straddling of the tricuspid valve

A congenital cardiac malformation in which there is a ventricula septal defect with exclusively muscular borders that opens into the inlet component of the right ventricle.

Defects within the muscular part of the ventricular septum that open to the inlet or the outlet of the right ventricle could also justifiably be considered to be within the apical part of the ventricular septum. More specific geographical codes, however, have been created for these latter variants. The code specifying defects within the trabecular part of the ventricular septum, therefore, should not be used to code the inlet or outlet muscular defects. The old code for "Type 4" defects would include all these defects with exclusively muscular borders

A congenital cardiac malformation in which there is a ventricul
septal defect within the trabeculated component of the ventricular septum.

Defects within the muscular part of the ventricular septum that open to the inlet or the outlet of the right ventricle could also justifiably be considered to be within the apical part of the ventricular septum. However these codes specifying defects within the trabecular part of the ventricular septum should not be used to code the inlet or outlet muscular defects as more specific geographical codes have been created for these latter variants

07.11.04 A congenital cardiac malformation in which there is a ventricular Muscular trabecular ventricular septal defect: Midseptal; septal defect that opens to the right ventricle in the middle midmuscular ventricular septal defect; trabecular muscular of the trabeculated component of the ventricular septum A congenital cardiac malformation in which there is a ventricula septal defect that opens to the right ventricle in the apical region of the trabeculated component of the ventricular septum ventricular septal defect with extension to right ventricular inlet (posterior)

Perimembranous inlet ventricular septal defect with atrioventricular septal malalignment; perimembranous ventricular septal defect with extension to right ventricular inlet (posterior) and atrioventricular septal malalignment; inlet perimembranous ventricular septal defect with atrioventricular septal malalignment; perimembranous with atrioventricular septal malalignment

Muscular inlet ventricular septal defect; muscular ventricular septal defect opening into right ventricular inlet; muscular ventricular septal defect in inlet septum

Muscular trabecular ventricular septal defect; type 4 ventricular septal defect; trabecular ventricular septal defect

\section{ventricular septal defect: mid}

Muscular trabecular ventricular septal defect: Apical; apical muscular ventricular septal defect 
ICD-11 congenital cardiology terms with

International Paediatric and Congenital

Cardiac Code (IPCCC) numbers, definitions and synonyms

Trabecular muscular ventricular septal defect: postero-inferior

07.11 .12

A congenital cardiac malformation in which there is a ventricular septal defect that opens to the right ventricle in the posteroinferior region of the trabeculated component of the ventricular septum.

It can be arbitrary as to when an inlet muscular defect becomes an inferior apical defect. The distinction should be made on whether or not the defect is partly shielded by the septal leaflet of the tricuspid valve

Trabecular muscular ventricular septal defect: $\quad 07.11 .07$ anterosuperior

Trabecular muscular ventricular septal defect: multiple

Outlet ventricular septal defect

Outlet ventricular septal defect without malalignment

07.12 .09

Outlet muscular ventricular septal defect without 07.11 .06 malalignment

Doubly committed juxta-arterial ventricular septal $\quad 07.12 .0$ defect without malalignment

Doubly committed juxta-arterial ventricular septal $\quad 07.12 .02$ defect without malalignment and with muscular postero-inferior rim
A congenital cardiac malformation in which there is a muscular anterior to the body of the septomarginal trabeculation, or septal band

A congenital cardiac malformation in which there are multiple muscular ventricular septal defects that open to the right ventricle in the trabeculated component of the ventricular septum

A congenital cardiac malformation in which there is a ventricular septal defect that opens to the outlet of the right ventricle between or above the limbs of the septal band

A congenital cardiac malformation in which there is an outlet ventricular septal defect, in the absence of malalignment of the outlet septum with the trabecular muscular septum

A congenital cardiac malformation in which there is an outlet ventricular septal defect with exclusively muscular borders, in the absence of malalignment of the outlet septum with the trabecular muscular septum

A congenital cardiac malformation in which there is an outlet ventricular septal defect bordered superiorly by the area of fibrous continuity or a fibrous outlet septum between the aortic and pulmonary valves, that is aligned with the trabecular muscular septum

A congenital cardiac malformation in which there is an outlet ventricular septal defect with muscular postero-inferior rim bordered superiorly by the area of fibrous continuity or a fibrous outlet septum between the aortic and pulmonary valves, that is aligned with the trabecular muscular septum
Muscular trabecular ventricular septal defect: postero-inferior; postero-inferior muscular ventricular septal defect; posterior muscular ventricular septal defect; inferior muscular ventricular septal defect; muscular ventricular septal defect in posteroinferior trabecular septum

Muscular trabecular ventricular septal defect: Anterosuperior anterosuperior muscular ventricular septal defect; anterior muscular ventricular septal defect; superior muscular ventricular septal defect; muscular ventricular septal defect opening anterior to body of septomarginal trabeculation

Multiple muscular trabecular ventricular septal defects; muscula trabecular ventricular septal defect: multiple; "Swiss cheese" septum

Supracristal ventricular septal defect; conal septal defect; infundibular ventricular septal defect; type 1 ventricular septa defect; subpulmonary ventricular septal defect; subarterial ventricular septal defect; juxta-arterial ventricular septal defect; conal ventricular septal defect; conoseptal hypoplasia;

intraconal ventricular septal defect

Muscular outlet ventricular septal defect; muscular outlet ventricular septal defect with aligned outlet septum; conal septal hypoplasia with exclusively muscular borders

Doubly committed subarterial ventricular septal defect; conoseptal hypoplasia with aligned outlet septum; doubly committed subarterial ventricular septal defect with aligned outlet septum; doubly committed juxta-arterial outlet ventricular septal defect; doubly committed subarterial outle ventricular septal defect; outlet ventricular septal defect with ligned outlet septum, juxta-arterial

Doubly committed juxta-arterial ventricular septal defect with muscular postero-inferior rim; doubly committed subarterial ventricular septal defect with muscular postero-inferior rim 
Doubly committed juxta-arterial ventricular septal $\quad 07.12 .03$ defect without malalignment and with

perimembranous extension

Outlet ventricular septal defect with anteriorly 07.10 .17 malaligned outlet septum

Outlet muscular ventricular septal defect with anteriorly malaligned outlet septum

Outlet perimembranous ventricular septal defect with anteriorly malaligned outlet septum

Doubly committed juxta-arterial ventricular septal $\quad 07.12 .12$ defect with anteriorly malaligned fibrous outlet septum

Doubly committed juxta-arterial ventricular septal $\quad 07.12 .07$ defect with anteriorly malaligned fibrous outlet septum and muscular postero-inferior rim

Doubly committed juxta-arterial ventricular septal 07.12 .0 defect with anteriorly malaligned fibrous outlet septum and perimembranous extension
A congenital cardiac malformation in which there is an outlet ventricular septal defect with perimembranous extension, bordered superiorly by the area of fibrous continuity or a fibrous outlet septum between the aortic and pulmonary valves, that is aligned with the trabecular muscular septum

Doubly committed juxta-arterial ventricular septal defect without malaligned fibrous outlet septum and with fibrous postero-inferior rim (perimembranous); doubly committed subarterial ventricular septal defect with perimembranous extension; doubly committed subarterial ventricular septal defect with aligned outlet septum with perimembranous extension; conal septal hypoplasia without cranial muscula border with perimembranous extension; doubly committed juxta-arterial outlet ventricular septal defect with

perimembranous extension

Ventricular septal defect opening into right ventricular outlet with anterior malalignment of outlet septum; outlet ventricular septal defect, Fallot type; Eisenmenger type ventricular septal ventricular septal defect and the muscular outlet septum is malaligned in an antero-cranial fashion with respect to the trabecular muscular septum such that there is overriding of the arterial valve supported predominantly by the left ventricle

A congenital cardiac malformation in which there is an outlet ventricular septal defect with exclusively muscular borders, and the muscular outlet septum is malaligned in an anterocranial fashion with respect to the trabecular muscular septum such that there is overriding of the arterial valve supported predominantly by the left ventricle

A congenital cardiac malformation in which there is an outle ventricular septal defect with perimembranous extension and the muscular outlet septum is malaligned in an anterocranial fashion with respect to the trabecular muscular septum such that there is overriding of the arterial valve supported predominantly by the left ventricle

A congenital cardiac malformation in which there is an outlet ventricular septal defect bordered superiorly by the area of fibrous continuity or a fibrous outlet septum between the aortic and pulmonary valves, and the fibrous outlet septum is malaligned in an antero-cranial fashion with respect to the trabecular muscular septum such that there is overriding of the arterial valve supported predominantly by the left ventricle

A congenital cardiac malformation in which there is an outlet ventricular septal defect with muscular postero-inferior rim bordered superiorly by the area of fibrous continuity or a fibrous outlet septum between the aortic and pulmonary valves, and the fibrous outlet septum is malaligned in an antero-cranial fashion with respect to the trabecular muscular septum such that there is overriding of the arterial valve supported predominantly by the left ventricle

A congenital cardiac malformation in which there is an outlet ventricular septal defect with perimembranous extension, bordered superiorly by the area of fibrous continuity or a fibrous outlet septum between the aortic and pulmonary valves, and the fibrous outlet septum is malaligned in an antero-cranial fashion with respect to the trabecular muscular septum such that there is overriding of the arterial valve supported predominantly by the left ventricle

Muscular outlet ventricular septal defect with anteriorly malaligned outlet septum; muscular ventricular septal defect opening into right ventricular outlet with anterior malalignment of outlet septum; muscular outlet ventricular septal defect, Fallot type

Perimembranous outlet ventricular septal defect with anteriorly malaligned outlet septum; perimembranous ventricular septa defect with outlet extension and anterior malalignment of outlet septum; perimembranous outlet ventricular septal defect, Fallot type

Doubly committed subarterial ventricular septal defect and anteriorly malalignment of outlet septum; doubly committed juxta-arterial ventricular septal defect "Fallot type"

Do

Doubly committed subarterial ventricular septal defect with

muscular posterior inferior rim and anteriorly malalignment of outlet septum; doubly committed juxta-arterial ventricular septal defect with muscular posterior inferior rim, "Fallot type"

Doubly committed juxta-arterial ventricular septal defect with anteriorly malaligned fibrous outlet septum and fibrous postero-inferior rim (perimembranous); doubly committed subarterial ventricular septal defect with perimembranous extension and anterior malalignment of outlet septum 
ICD-11 congenital cardiology terms with

International Paediatric and Congenital

Cardiac Code (IPCCC) numbers, definitions and synonyms

\section{IPCCC code Definition with commentar}

07.10 .18

malaligned outlet septum

Outlet muscular ventricular septal defect with posteriorly malaligned outlet septum

07.11 .16

Outlet perimembranous ventricular septal defect with posteriorly malaligned outlet septum

Doubly committed juxta-arterial ventricular septal $\quad 07.12 .13$ defect with posteriorly malaligned fibrous outlet septum

Doubly committed juxta-arterial ventricular septal $\quad 07.12 .08$ defect with posteriorly malaligned fibrous outlet septum and muscular postero-inferior rim

Doubly committed juxta-arterial ventricular septal $\quad 07.12 .06$ defect with posteriorly malaligned fibrous outlet septum and perimembranous extension
A congenital cardiac malformation in which there is an outlet ventricular septal defect and the muscular outlet septum is malaligned in a postero-caudal fashion with respect to the trabecular muscular septum such that there is usually

obstruction to the left ventricular outflow tract

A congenital cardiac malformation in which there is an outlet ventricular septal defect with exclusively muscular borders, and the muscular outlet septum is malaligned in a posterocaudal fashion with respect to the trabecular muscular septum such that there is usually obstruction to the left ventricular outflow tract

A congenital cardiac malformation in which there is an outle ventricular septal defect with perimembranous extension and the muscular outlet septum is malaligned in a posterocaudal fashion with respect to the trabecular muscular septum such that there is usually obstruction to the left ventricular outflow tract

A congenital cardiac malformation in which there is an outlet ventricular septal defect bordered superiorly by the area of fibrous continuity or a fibrous outlet septum between the aortic and pulmonary valves, and the fibrous outlet septum is malaligned in a postero-caudal fashion with respect to the trabecular muscular septum such that there is usually obstruction to the left ventricular outflow tract

A congenital cardiac malformation in which there is an outlet ventricular septal defect with muscular postero-inferior rim, bordered superiorly by the area of fibrous continuity or a fibrous outlet septum between the aortic and pulmonary valves, and the fibrous outlet septum is malaligned in a postero-caudal fashion with respect to the trabecular muscular septum such that there is usually obstruction to the left ventricular outflow tract

A congenital cardiac malformation in which there is an outlet ventricular septal defect with perimembranous extension, bordered superiorly by the area of fibrous continuity or a fibrous outlet septum between the aortic and pulmonary valves, and the fibrous outlet septum is malaligned in a postero-caudal fashion with respect to the trabecular muscular septum such that there is usually obstruction to the left ventricular outflow tract
Synonyms

Ventricular septal defect opening into right ventricular outlet with posterior malalignment of outlet septum; outlet ventricular septal defect, "coarctation type"

Muscular ventricular septal defect opening into right ventricular outlet with posterior malalignment of outlet septum; muscular outlet ventricular septal defect "coarctation type"; muscula outlet ventricular septal defect with posteriorly malaligned outlet septum

Perimembranous outlet ventricular septal defect with posteriorly malaligned outlet septum; perimembranous ventricular septal defect with outlet extension and posterior malalignment of outlet septum; perimembranous outlet ventricular septal defect, coarctation type

Doubly committed subarterial ventricular septal defect with posterior malalignment of outlet septum; doubly committed juxta-arterial ventricular septal defect, coarctation type

Doubly committed subarterial ventricular septal defect with muscular posterior inferior rim and posterior malalignment of outlet septum; doubly committed juxta-arterial ventricular septal defect with muscular posterior inferior rim, "coarctation type

Doubly committed juxta-arterial ventricular septal defect with posteriorly malaligned fibrous outlet septum and fibrous postero-inferior rim (perimembranous); doubly committed subarterial ventricular septal defect with perimembranous extension and posterior malalignment of outlet septum; doubly committed juxta-arterial ventricular septal defect with perimembranous extension, coarctation type 
Ventricular septal defect: haemodynamically insignificant

Multiple ventricular septal defects

07.15 .04

Functionally univentricular heart

Double inlet atrioventricular connection

Double inlet left ventricle

01.04 .04

Double inlet right ventricle

07.15 .01
A congenital cardiac malformation in which there is one or more Maladie de Roger, Restrictive ventricular septal defect small, clinically insignificant ventricular septal defect(s) in the absence of flow-related cardiac chamber dilation or abnormal elevation of pulmonary arterial pressure.

Though restrictive ventricular septal defect is listed as a synonym of baemodynamically insignificant ventricular septal defect, it should be recognised that some pressure restrictive ventricular septal defects will lead to flow-related chamber dilation, and thus are would be baemodynamically significant. In such instances, the term

baemodynamically insignificant ventricular septal defect should not be coded

A congenital cardiac malformation in which there are multiple ventricular septal defects, which could be of any type

The term "functionally univentricular heart" describes a

spectrum of congenital cardiac malformations in which the ventricular mass may not readily lend itself to partitioning that commits one ventricular pump to the systemic circulation, and another to the pulmonary circulation.

A beart may be functionally univentricular because of its anatomy or because of the lack of feasibility or lack of advisability of surgically partitioning the ventricular mass. Common lesions in this category typically include double inlet right ventricle, double inlet left ventricle, tricuspid atresia, mitral atresia, and hypoplastic left beart syndrome. Other lesions which sometimes may be considered to be a functionally univentricular beart include complex forms of atrioventricular septal defect, double outlet right ventricle, congenitally corrected transposition, pulmonary atresia with intact ventricular septum, and other cardiovascular malformations.

Specific diagnostic codes should be used whenever possible, and not the term "functionally univentricular beart"

A congenital cardiovascular malformation with a univentricular Double inlet ventricle atrioventricular connection wherein both atria connect to one ventricle either via two separate atrioventricular valves or a common atrioventricular valve, such that all or nearly all of the total atrioventricular junctional (annular) area is committed to one ventricular chamber

A congenital cardiovascular malformation with a univentricular atrioventricular connection wherein both atria connect to a morphologically left ventricle either via two separate atrioventricular valves or a common atrioventricular valve, such that all or nearly all of the total atrioventricular junctional (annular) area is committed to the left ventricular chamber

A congenital cardiovascular malformation with a univentricular atrioventricular connection wherein both atria connect to a morphologically right ventricle either via two separate atrioventricular valves or a common atrioventricular valve, such that all or nearly all of the total atrioventricular junctional (annular) area is committed to the right ventricular chamber
Univentricular cardiopathy; single ventricle; univentricular heart

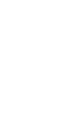


ICD-11 congenital cardiology terms with

International Paediatric and Congenital

Cardiac Code (IPCCC) numbers, definitions

and synonyms

Double inlet to solitary ventricle of indeterminate 01.04 .05 morphology

Tricuspid atresia

06.01 .01

Tricuspid atresia with absent valvar annulus

06.01 .26

Tricuspid atresia with imperforate tricuspid valve 06.01 .02

Mitral atresia

06.02 .01

Mitral atresia with absent valvar annulus

Mitral atresia with imperforate mitral valve

06.02 .02

Hypoplastic left heart syndrome

01.01 .09

Congenital anomaly of a ventriculo-arterial valve

09.04 .29

or adjacent regions

Congenital anomaly of pulmonary valve

Congenital pulmonary valvar stenosis

09.05 .04
A congenital cardiovascular malformation with a univentricular atrioventricular connection wherein both atria connect to a solitary and morphologically indeterminate ventricle either via two separate atrioventricular valves or a common atrioventricular valve

A congenital cardiovascular malformation with absence of the tricuspid valvar annulus (connection/junction) or an imperforate tricuspid valve

A congenital cardiovascular malformation with absence of the tricuspid valve and its associated annulus (connection/junction)

A congenital cardiovascular malformation with an imperforate tricuspid valve (the tricuspid valve and associated atrioventricular connection/junction are present but the valve is imperforate)

A congenital cardiovascular malformation with absence of the mitral valvar annulus (connection/junction) or an imperforate mitral valve

A congenital cardiovascular malformation with absence of the mitral valve and its associated annulus (connection/junction)

A congenital cardiovascular malformation with an imperforate mitral valve (the mitral valve and associated atrioventricula connection/junction are present but the valve is imperforate).

A spectrum of congenital cardiovascular malformations with normally aligned great arteries without a common atrioventricular junction, characterised by underdevelopment of the left heart with significant hypoplasia of the left ventricle including atresia, stenosis, or hypoplasia of the aortic or mitral valve, or both valves, and hypoplasia of the ascending aorta and aortic arch

A congenital cardiovascular malformation of a ventriculo-arteria valve or its immediate subvalvar and supravalvar regions. This does not include anomalies of the truncal valve which are classified along with codes related to the common arterial trunk

A congenital malformation of the heart where the pulmonary valve is abnormal

A congenital cardiovascular malformation of the pulmonary valve which there is narrowing or stricture (obstruction to flow). Congenital pulmonary valvar stenosis ranges from critical neonatal pulmonic valve stenosis with hypoplasia of the right ventricle to valvar
Tricuspid atresia, absent right atrioventricular connection or junction

Hypoplasia of the left heart; HLHS - [hypoplastic left heart syndrome]; left heart hypoplasia syndrome

Congenital pulmonary stenosis; congenital pulmonary valve stricture; congenital stenosis of pulmonary valve 
Pulmonary 'annular' hypoplasia

09.05 .05

Congenital pulmonary regurgitation

09.05 .22

Dysplasia of pulmonary valve

09.05 .24

Bicuspid pulmonary valve

Congenital subpulmonary stenosis

09.05 .32

07.05 .30

Congenital supravalvar pulmonary stenosis

09.07 .15

Congenital pulmonary atresia

09.05 .16

Pulmonary atresia with intact ventricular septum $\quad 01.01 .07$ pulmonary stenosis in the infant, child, or adult, usually better tolerated but potentially associated with infundibular stenosis. Pulmonary branch bypoplasia can be associated. Only $10 \%$ of neonates with this and intact ventricular septum bave right ventricle-to-coronary artery fistula(s). An right ventricle dependent coronary artery circulation is present when coronary artery fistulas (coronary sinusoids) are associated with a proximal coronary artery stenosis; this occurs in only $2 \%$ of neonates with pulmonary valvar stenosis and an intact ventricular septum

A congenital cardiovascular malformation of the pulmonary valve in which its "annulus" is hypoplastic (incomplete development or underdevelopment so that it is abnormally small [below the lower limit of normal adjusted for body size])

Congenital cardiovascular malformation of the pulmonary valve Congenital pulmonary insufficiency; congenital pulmonary allowing backward flow into the ventricle.

Congenital pulmonary valve regurgitation may be due to primary annular dilation, prolapse, and leaflet underdevelopment

A congenital cardiovascular malformation where the pulmonary Pulmonary valve dysplasia; dysplastic pulmonary valve; valve leaflets are markedly thickened with restricted mobility, pulmonary valve cusp dysplasia characterised by the presence of myxomatous tissue

A congenital cardiovascular malformation where the pulmonary Bicommisural pulmonary valve valve has only two leaflets (cusps), and includes absent or fused commissure

A congenital cardiovascular malformation associated with Infundibular pulmonary stenosis narrowing within the outflow tract supporting the pulmonary valve.

Subvalvar (infundibular) pulmonary stenosis is a narrowing of the outflow tract of the ventricle below the pulmonic valve. It may be due to a localised fibrous diaphragm just below the valve, an obstructing muscle bundle, or to a long narrow fibromuscular channel.

Subvalvar pulmonary stenosis is a cause of right ventricular outflow tract obstruction if the ventriculo-arterial connections are normal and should be differentiated from double-chambered right ventricle where obstruction is at the mid-chamber level

A congenital cardiovascular malformation associated with narrowing at the level of the pulmonary sinotubular junction

A congenital cardiovascular malformation in which there is no Pulmonary atresia opening between any ventricle and the pulmonary arterial tree.

For "pulmonary atresia with VSD", please see the section under "Tetralogy of Fallot"

A congenital cardiovascular malformation in which there are normally aligned great arteries, no opening between the morphologically right ventricle and the pulmonary trunk, and no ventricular level communication.

Pulmonary atresia with intact ventricular septum is a duct-dependent congenital malformation that forms a spectrum of lesions including atresia of the pulmonary valve, a varying degree of right ventricle and tricuspid valve hypoplasia, and anomalies of the coronary circulation. A right ventricular dependent coronary artery circulation is present when coronary artery fistulas (coronary 
ICD-11 congenital cardiology terms with

International Paediatric and Congenital

Cardiac Code (IPCCC) numbers, definitions

and synonyms

IPCCC code Definition with commentar

Synonyms

Congenital anomaly of aortic valve

Congenital aortic valvar stenosis

Congenital aortic regurgitation

09.15 .07 sinusoids) are associated with a proximal coronary artery stenosis. Associated Ebstein anomaly of the tricuspid valve can be present; the tricuspid diameter is enlarged and the prognosis is poor

A congenital cardiovascular malformation where the aortic valve is abnormal

A congenital cardiovascular malformation of the aortic valve in which there is narrowing or stricture (obstruction to flow). Congenital valvar aortic stenosis and/or hypoplasia is of two types: critical (infantile), seen in the newborn in whom systemic perfusion depends on a patent ductus arteriosus, and non-critical, seen in infancy or later. It may result: (1) from complete fusion of commissures that results in a dome-shaped valve with a pinpoint opening (seen most commonly in infants with critical aortic valve stenosis): (2) from a unicommissural valve with one defined commissure and eccentric orifice (often with two raphes radiating from the ostium indicating underdeveloped commissures of a tricuspid aortic valve); (3) from a bicuspid aortic valve, with leaflets that can be equal in size or discrepant, and in left-right or anterior-posterior position; and finally; (4) from a dysplastic tricuspid valve, which may have a gelatinous appearance with thick rarely equal in size leaflets, often obscuring the commissures. The dysplastic, tricuspid, or bicuspid form of aortic valve deformity may not be initially obstructive but may become stenotic later in life due to leaflet thickening and calcification

Congenital cardiovascular malformation of the aortic valve allowing backward flow into the ventricle.

Congenital aortic regurgitation/insufficiency is rare as an isolated entity. There are rare reports of congenital malformation of the aortic valve that result in aortic insufficiency shortly after birth from an absent or underdeveloped aortic valve cusp. Aortic insufficiency is more commonly seen with other associated cardiovascular anomalies: (1) in stenotic aortic valves (commonly stenotic congenital bicuspid aortic valves) with some degree of aortic regurgitation due to aortic leaflet abnormality; (2) in association with a ventricular septal defect (especially in subarterial type and is commonly seen in Asian populations): (3) secondary to aortic-left ventricular tunnel; (4) secondary to tethering or retraction of aortic valve leaflets in cases of supravalvar aortic stenosis that may involve the aortic valve; and similarly; (5) secondary to encroachment on an aortic cusp by a subaortic fibromuscular ridge; or (6) turbulence caused by a stenotic jet can create progressive aortic regurgitation
Congenital valvar aortic stenosis; congenital valvular aortic stenosis; congenital aortic valve stricture; congenital stenosis of aortic valve; congenital aortic valve stenosis

Congenital aortic incompetence; congenital aortic insufficiency; congenital insufficiency of aortic valve 
Bicuspid aortic valve

09.15 .22

Unicuspid aortic valve

09.15 .21

Aortic valvar prolapse

09.15 .30

Aortic valvar atresia

Aortic 'annular' hypoplasia

Dysplasia of aortic valve

Congenital subaortic stenosis

Subaortic stenosis due to fibromuscular shelf

07.09 .03

Subaortic stenosis due to fibromuscular tunnel

07.09 .16

Congenital supravalvar aortic stenosis
A congenital cardiovascular malformation where the aortic valve Bicommisural aortic valve has two commissures and two separate leaflets (cusps)

because of fusion or absence of one of the commissures

A congenital cardiovascular malformation in which the aortic Unicommissural aortic valve valve has a single commissure and a single or functionally single leaflet (cusp).

A congenital cardiovascular malformation of the aortic valve in Aortic valve prolapse

which part or all of one or more of the aortic valve leaflets is on the ventricular side of the plane of the inferior aspect of the attachments of the aortic valve leaflets.

NB: This is classified under Heart Valve Disease in ICD-11 within the acquired sections as the lesion is rarely truly congenital and present at birth

A congenital cardiovascular malformation in which there is no orifice of the aortic valve.

Aortic valve atresia will most often be coded under the bypoplastic left heart syndrome/complex diagnostic codes since it most often occurs as part of a spectrum of cardiovascular malformations. However, there is a small subset of patients with aortic valve atresia who have a well developed left ventricle and mitral valve and a large ventricular septal defect (non-restrictive or restrictive)

A congenital cardiovascular malformation of the aortic valve in Hypoplasia of the aortic annulus which its "annulus" is hypoplastic (incomplete development or underdevelopment so that it is abnormally small [below the lower limit of normal adjusted for body size])

09.15.09 A congenital cardiovascular malformation where the aortic valve Aortic valve cusp dysplasia; aortic valvar dysplasia leaflets are markedly thickened with restricted mobility,

characterised by the presence of myxomatous tissue

A congenital cardiovascular malformation associated with

Congenital subvalvular aortic stenosis; congenital subvalvar narrowing within the outflow tract supporting the aortic valve. aortic stenosis

Subaortic obstruction can be caused by different lesions: subaortic

membrane or tunnel, accessory mitral valve tissue, abnormal insertion of

the mitral anterior leaflet to the ventricular septum, deviation of the outlet septum (seen in coarctation of the aorta and interrupted aortic arch), or a restrictive ventricular septal defect (bulboventricular foramen) in single ventricle complexes. Subvalvar aortic stenosis may be categorised into two types: localised subvalvar aortic stenosis, which consists of a fibrous or fibromuscular ridge, and diffuse tunnel subvalvar aortic stenosis, in which circumferential narrowing commences at the annular level and extends downward for 1-3 cm

A congenital cardiovascular malformation in which there is subaortic Discrete left ventricular outflow tract obstruction stenosis due to a discrete fibrous and/or muscular ridge

A congenital cardiovascular malformation in which there is a long-segment fibrous and/or muscular subaortic stenosis

09.16.18 A congenital cardiovascular malformation with narrowing of the aorta at the level of the sinotubular junction which may extend into the ascending aorta.

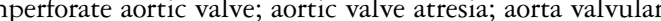
atresia; congenital atresia of aortic valve 
ICD-11 congenital cardiology terms with

International Paediatric and Congenital

Cardiac Code (IPCCC) numbers, definitions

and synonyms

Aneurysm of aortic sinus of Valsalva

09.18 .01

Aortoventricular tunnel

09.17 .01
Congenital supravalvar aortic stenosis is described as three forms: an hourglass deformity, a fibrous membrane, and a diffuse narrowing of the ascending aorta. The disease can be inherited as an autosomal dominant trait or part of Williams-Beuren syndrome in association with intellectual disability, elfin facies, failure to thrive, and occasionally infantile bypercalcemia. Supravalvar aortic stenosis may involve the coronary artery ostia, and the aortic leaflets may be tethered. The coronary arteries can become tortuous and dilated due to elevated pressures and early atherosclerosis may ensue

A congenital cardiovascular malformation in which there is dilation of a single aortic sinus of Valsalva.

The sinus of Valsalva is defined as that portion of the aortic root between the aortic root annulus and the sinotubular ridge. A congenital sinus of Valsalva aneurysm is a dilation usually of a single sinus of Valsalva. These most commonly originate from the right sinus (65-85\%), less commonly from the non-coronary sinus $(10-30 \%)$, and rarely from the left sinus $(<5 \%)$. A true sinus of Valsalva aneurysm presents above the aortic annulus. The hierarchical coding system distinguishes between congenital versus acquired, ruptured versus non-ruptured, sinus of origin, and chamber/site of penetration (right atrium, right ventricle, left atrium, left ventricle, pulmonary artery, pericardium). A nonruptured congenital sinus of Valsalva aneurysm may vary from a mild dilation of a single aortic sinus to an extensive windsock deformity. Rupture of a congenital sinus of Valsalva aneurysm into an adjacent chamber occurs most commonly between the ages of 15 and 30 years. Rupture may occur spontaneously, after trauma, after strenuous physical exertion, or from acute bacterial endocarditis.

Congenital aetiology is supported by the frequent association of sinus of Valsalva aneurysms with ventricular septal defects. This is to be distinguished from aortic root dilation associated with connective tissue disorders

A congenital cardiovascular malformation in which there is a paravalvar communication between the aorta and a ventricle.

The aorto-left ventricular tunnel is an abnormal paravalvar (alongside or in the vicinity of a valve) communication between the aorta and left ventricle, commonly divided into four types: type I, a simple tunnel with a slit-like opening at the aortic end and no aortic valve distortion; type II, a large extracardiovascular aortic wall aneurysm of the tunnel with an oval opening at the aortic end, with
Sinus of Valsalva aneurysm

Aortico-ventricular tunnel; ventriculo-aortic tunnel 
Congenital anomaly of great arteries including arterial duct

Congenital aortopulmonary window

Congenital anomaly of pulmonary arterial tree

Congenital dilation of pulmonary arterial tree

Congenital pulmonary trunk anomaly

Congenital pulmonary trunk hypoplasia

Absent or atretic pulmonary trunk

Congenital pulmonary arterial branch anomaly

Congenital pulmonary arterial branch stenosis

Congenital right pulmonary arterial stenosis
09.04 .28

09.04 .07

09.07 .16

09.10 .36

09.07 .19

09.07 .20

09.07 .05

09.10 .41

09.10 .27

09.10 .28 or without ventricular distortion; type III, intracardiovascular aneurysm of the septal portion of the tunnel, with or without right ventricular outflow obstruction; and type IV, a combination of types II and III. Further differentiation within these types may be notation of right coronary artery arising from the wall of the tunnel. If the tunnel communicates with the right ventricle, many feel that the defect is really a ruptured sinus of Valsalva aneurysm

A congenital cardiovascular malformation of the great arteries (aorta, pulmonary trunk [main pulmonary artery], branch pulmonary arteries) or the arterial duct (ductus arteriosus). This excludes the truncal root seen with common arterial trunk

A congenital cardiovascular malformation in which there is side- Aortopulmonary window; aortic septal defect; aortopulmonary to-side continuity of the lumens of the ascending aorta and septal defect; aorticopulmonary window; aorticopulmonary pulmonary trunk in association with separate aortic and pulmonary valves or their atretic remnants.

Side-to-side continuity of the lumens of the aorta and pulmonary arterial tree, which is distinguished from common arterial trunk (truncus arteriosus) by the presence of two arterial valves or their atretic remnants, and involvement of the pulmonary trunk (main pulmonary artery)

A congenital cardiovascular malformation of the pulmonary trunk (main pulmonary artery) and/or branch pulmonary arteries (right, left, and ramifications)

A congenital cardiovascular malformation in which there is an enlargement of the luminal diameter of the pulmonary trunk (main pulmonary artery) and/or branch pulmonary arteries (above the upper limit of normal adjusted for body size)

A congenital cardiovascular malformation of the pulmonary arterial trunk (main pulmonary artery)

A congenital cardiovascular malformation of the pulmonary trunk (main pulmonary artery) in which there is diffuse luminal narrowing (below the lower limit of normal adjusted for body size).

The stenosis or hypoplasia may be isolated or associated with other cardiovascular lesions. Since the narrowing is distal to the pulmonary valve, it may also be known as supravalvar pulmonary stenosis

A congenital cardiovascular malformation where the pulmonary Atretic pulmonary trunk; pulmonary trunk absent; absent main trunk (main pulmonary artery) is not present or has luminal pulmonary artery; atretic main pulmonary artery occlusion, excluding common arterial trunk

A congenital cardiovascular malformation of a pulmonary arterial Congenital abnormality of pulmonary artery branch

A congenital cardiovascular malformation in which there is discrete narrowing of the luminal diameter of one or more segments of a pulmonary arterial branch (below the lower limit of normal adjusted for body size). This implies an area of narrowing surrounded by non-stenotic lumen

A congenital cardiovascular malformation in which there is discrete narrowing of the luminal diameter of one or more segments of the right pulmonary artery (below the lower

Distal peripheral pulmonary stenosis right; peripheral limit of normal adjusted for body size)

Congenital main pulmonary artery anomaly

Main pulmonary artery hypoplasi 
ICD-11 congenital cardiology terms with

International Paediatric and Congenital

Cardiac Code (IPCCC) numbers, definitions

and synonyms

IPCCC code Definition with commentar

09.10 .29

A congenital cardiovascular malformation in which there is discrete narrowing of the luminal diameter of one or more segments of the left pulmonary artery (below the lower limit of normal adjusted for body size)

Congenital pulmonary arterial hypoplasia

Congenital right pulmonary arterial hypoplasia

09.10 .72

Congenital left pulmonary arterial hypoplasia

09.10 .73

Absent or atretic right pulmonary artery

Absent or atretic left pulmonary artery

09.10 .77

Congenital central pulmonary arterial stenosis or $\quad 09.10 .37$ hypoplasia proximal to hilar bifurcation

Congenital peripheral pulmonary arterial stenosis 09.10 .38 or hypoplasia at or beyond hilar bifurcation

Congenitally discontinuous, non-confluent right 09.10 .30 and left pulmonary arteries

Pulmonary artery origin from ascending aorta

09.09 .08 with other cardiovascular lesions. Coarctation of the pulmonary
A congenital cardiovascular malformation in which there is diffuse luminal narrowing of a pulmonary arterial branch (below the lower limit of normal adjusted for body size)

A congenital cardiovascular malformation in which there is diffuse luminal narrowing of the right pulmonary artery (below the lower limit of normal adjusted for body size)

A congenital cardiovascular malformation in which there is diffuse luminal narrowing of the left pulmonary artery (below the lower limit of normal adjusted for body size)

A congenital cardiovascular malformation in which the right pulmonary artery is not present or has luminal occlusion

A congenital cardiovascular malformation in which the left pulmonary artery is not present or has luminal occlusion

A congenital cardiovascular malformation of a pulmonary artery, proximal to its first branch, in which there is luminal narrowing (below the lower limit of normal adjusted for body size).

Central pulmonary artery branch (within the bilar bifurcation

involving the right or left pulmonary artery, or both) narrowing or bypoplasia. The stenosis or hypoplasia may be isolated or associated artery is related to abnormal extension of the ductus arteriosus into a pulmonary artery branch, more frequently the left branch

A congenital cardiovascular malformation of a pulmonary artery, Peripheral pulmonary stenosis; distal peripheral pulmonary distal to its first branch, in which there is luminal narrowing stenosis; peripheral pulmonary arterial stenosis; distal (below the lower limit of normal adjusted for body size).

Peripheral pulmonary artery narrowing or hypoplasia at or beyond the

bilar bifurcation. The stenosis or bypoplasia may be isolated or associated with other cardiovascular lesions

A congenital cardiovascular malformation in which there is absence of luminal continuity between the right and lef branch pulmonary arteries.

The discontinuous branch pulmonary artery is typically supplied by a patent ductus arteriosus or an aortopulmonary collateral

A congenital cardiovascular malformation in which one branch Hemitruncus pulmonary artery arises from the ascending aorta and the other branch pulmonary artery arises from the pulmonary trunk (main pulmonary artery) peripheral pulmonary arterial stenosis
Synonyms

Distal peripheral pulmonary stenosis left; peripheral pulmonary stenosis left

Hypoplasia of pulmonary artery; congenital hypoplasia of pulmonary artery; pulmonary artery hypoplasia

Distal peripheral pulmonary hypoplasia-right; peripheral pulmonary hypoplasia-right

Distal peripheral pulmonary hypoplasia left; peripheral pulmonary hypoplasia left

Atretic right pulmonary artery; absent right pulmonary artery

Atretic left pulmonary artery; absent left pulmonary artery

Central pulmonary arterial stenosis; central pulmonary stenosis; proximal pulmonary arterial stenosis; proximal pulmonary stenosis 
Right pulmonary artery from ascending aorta

09.09 .03

Left pulmonary artery from ascending aorta

09.09 .05

Pulmonary artery from arterial duct

Right pulmonary artery from arterial duct

Left pulmonary artery from arterial duct

Congenital anomaly of aorta or its branches

Congenital anomaly of ascending aorta

Ascending aortic hypoplasia

Congenital ascending aortic dilation or aneurysm

09.16 .19

Coarctation of aorta

09.29 .01

Congenital anomaly of aortic arch

Aortic arch hypoplasia
One pulmonary artery arises from the ascending aorta and the other pulmonary artery arises from the right ventricle. This does include origin of the right or left pulmonary artery from the innominate artery or the artic arch via a patent arterial duct or collateral artery

A congenital cardiovascular malformation in which the right pulmonary artery arises from the ascending aorta and the left pulmonary artery arises from the pulmonary trunk (main pulmonary artery)

A congenital cardiovascular malformation in which the left pulmonary artery arises from the ascending aorta and the right pulmonary artery arises from the pulmonary trunk (main pulmonary artery)

A congenital cardiovascular malformation in which the pulmonary arteries are non-confluent and one or both arise from an arterial duct (ductus arteriosus)

A congenital cardiovascular malformation in which the pulmonary Right pulmonary artery from ductus arteriosus arteries are non-confluent and the right pulmonary artery arises from an arterial duct (ductus arteriosus)

A congenital cardiovascular malformation in which the Left pulmonary artery from ductus arteriosus pulmonary arteries are non-confluent and the left pulmonary artery arises from an arterial duct (ductus arteriosus)

A congenital cardiovascular malformation of the aorta and/or its branches

A congenital cardiovascular malformation of the aorta between the sinotubular junction and the origin of its first branch

A congenital cardiovascular malformation in which the luminal diameter of the aorta between its sinotubular junction and the origin of its first branch is narrowed (below the lower limit of normal adjusted for body size)

A congenital cardiovascular malformation in which the luminal diameter of the aorta between its sinotubular junction and the origin of its first branch is dilated (above the upper limit of normal adjusted for body size)

A congenital cardiovascular malformation in which there is a discrete luminal narrowing of the junction between the aortic arch and the descending aorta.

Coarctation of the aorta generally indicates a narrowing of the

descending thoracic aorta just distal to the left subclavian artery. However, the term may also be accurately used to refer to a region of narrowing anywhere in the thoracic or abdominal aorta

A congenital cardiovascular malformation of the aorta between the origin of the innominate artery and the insertion of the arterial duct (ductus arteriosus)

This includes "persistent fifth aortic arch"

A congenital cardiovascular malformation in which there is diffuse luminal narrowing of the aortic arch (below the lower limit of normal adjusted for body size).

Hypoplasia of the aortic arch is hypoplasia of the proximal or distal transverse arch or the aortic isthmus. The isthmus (arch between the 
ICD-11 congenital cardiology terms with

International Paediatric and Congenital

Cardiac Code (IPCCC) numbers, definitions

and synonyms

left subclavian and insertion of the patent ductus arteriosus/

ligamentum arteriosum) is hypoplastic if its diameter is $<40 \%$ of

the diameter of the ascending aorta. The proximal transverse arch

(arch between the innominate and left carotid arteries) and distal

transverse arch (arch between the left carotid and left subclavian

arteries) are hypoplastic if their diameters are $<60$ and $50 \%$.

respectively, of the diameter of the ascending aorta

Interrupted aortic arch distal to subclavian artery, 09.29.32 type A

Interrupted aortic arch between subclavian and common carotid arteries, type B

Interrupted aortic arch between carotid arteries, $\quad 09.29 .34$ type $\mathrm{C}$

Right aortic arch

Left aortic arch

09.28 .22

A congenital cardiovascular malformation in which there is an absence of luminal continuity between the ascending and descending aorta.

This term includes luminal atresia with discontinuity between the aortic segments and also luminal atresia with fibrous continuity between the aortic segments. Interrupted aortic arch is defined as the loss of luminal continuity between the ascending and descending aorta. In most cases, blood flow to the descending thoracic aorta is through a patent arterial duct, and there is a large ventricular septal defect. Arch interruption is further defined by site of interruption. In type A, interruption is distal to the left subclavian artery; in type $B$, interruption is between the left carotid and left subclavian arteries; and in type $C$, interruption occurs between the innominate and left carotid arteries there is an absence of luminal continuity distal to the subclavian artery ipsilateral to the arch

A congenital cardiovascular malformation of the aorta in which Aortic arch interruption, type B; aortic interruption, type B; there is an absence of luminal continuity of the aorta between the carotid and subclavian arteries

congenital cardiovascular malformation of the aorta in which there is an absence of luminal continuity of the aorta between the carotid arteries

A congenital cardiovascular malformation of the great vessels in Right arch; right-sided arch; right-sided aortic arch; persisten which the aortic arch crosses to the right of the trachea

Interruption of the aortic arch; aortic arch interruption; aortic atresia

right arch; persistent right-sided arch; persistent right-sided aortic arch

A congenital cardiovascular finding of the great vessels in which Left arch; left-sided arch; left-sided aortic arch the aortic arch crosses to the left of the trachea.

To be coded only when this represents an abnormal finding, such as in situs inversus

A congenital cardiovascular malformation in which the aortic Cervical arch arch is located superior to the clavicle, and is most commonly right sided 
Congenital anomaly of aortic arch branch

09.30 .17

Aberrant origin of right subclavian artery

09.30 .02

Aberrant origin of left subclavian artery

Isolation of an aortic arch branch

Congenital anomaly of descending thoracic or abdominal aorta

Descending thoracic or abdominal aortic coarctation

Tracheo-oesophageal compressive syndrome

Innominate artery compression syndrome

Vascular ring

09.31 .00

Double aortic arch

Vascular ring: right aortic arch and left arterial duct or ligament

Vascular ring: left aortic arch and right arterial duct or ligament

09.30 .04

09.29 .44

09.30 .23

09.31 .34
A congenital cardiovascular malformation of one or more branches of the aortic arch (innominate, carotid, or subclavian arteries)

A congenital cardiovascular malformation in which the right the setting of a left aortic arch

A congenital cardiovascular malformation in which the left subclavian artery arises distal to the right subclavian artery artery in the setting of a right arch

09.30.16 A congenital cardiovascular malformation in which the only connection to a brachiocephalic vessel is via an arterial duct (ductus arteriosus) or its ligament.

This includes isolation of the innominate artery, left common carotid artery, right common carotid artery and either subclavian artery

A congenital cardiovascular malformation of the aorta dist the aortic arch

A congenital cardiovascular malformation in which there is luminal narrowing of the lower thoracic or abdominal aorta

09.31.40 A congenital cardiovascular malformation which causes compression of the trachea and/or the oesophagus

A congenital cardiovascular malformation in which there is compression of the trachea by the innominate artery.

This syndrome is a true compression of the trachea by the abnormally positioned innominate artery. The innominate artery can appear to "compress" the trachea in the presence of tracheomalacia but, in the absence of an abnormal origin and course of the innominate artery, this apparent "compression" most likely is a consequence of the tracheomalacia itself rather than actual compression by the innominate artery

A congenital cardiovascular malformation in which one or more of the following encircle the trachea and oesophagus: the aorta and its major branches, the pulmonary trunk and its major branches, and the arterial duct (ductus arteriosus) or their vascular remnant(s).

The term vascular ring refers to a group of congenital vascular anomalies that encircle with the potential to compress the oesophagus and-or trachea. The compression may be from a complete anatomic ring (double aortic arch or right aortic arch with a left ligamentum) or from a compressive effect of an aberrant vessel (innominate artery compression syndrome)

09.28.09 A congenital cardiovascular malformation in which the right and Encircling double aortic arch left aortic arches (patent or atretic) encircle the trachea and oesophagus

09.31.35 A congenital cardiovascular malformation in which continuity of the right aortic arch, pulmonary trunk and left arterial duct (or ligament) encircles the trachea and oesophagus.

This code is inclusive of with or without diverticulum of Kommerell

A congenital cardiovascular malformation in which continuity of the left aortic arch, pulmonary trunk and right arterial duct (or ligament) encircles the trachea and oesophagus.

This code is inclusive of with or without diverticulum of Kommerell
Congenital anomaly of thoraco-abdominal aorta

Middle aortic syndrome

Innominate artery compressive syndrome; Brachiocephalic arter compression syndrome; brachiocephalic artery compressive syndrome; innominate artery compression of the trachea; brachiocephalic artery compression of the trachea; aberrant innominate artery; aberrant brachiocephalic artery; anomalous origin of the innominate artery; anomalous origin of the brachiocephalic artery

\author{
(n)
}


ICD-11 congenital cardiology terms with

International Paediatric and Congenital

Cardiac Code (IPCCC) numbers, definitions

and synonyms

\section{IPCCC code Definition with commentar}

Anomalous origin of left pulmonary artery from right pulmonary artery

Congenital arterial duct anomaly

Patent arterial duct

Systemic-to-pulmonary collateral arteries

09.08 .18

Congenital anomaly of coronary arteries

09.46 .03
A congenital cardiovascular malformation in which the left pulmonary artery originates from the right pulmonary artery and passes between the trachea and oesophagus, and is often associated with tracheobronchial anomalies such as tracheomalacia, stenosis, or complete tracheal rings

A congenital cardiovascular malformation of the arterial duct (ductus arteriosus) or its fibrous remnant (ligamentum arteriosum).

The described anomalies include an anomalous course, abnormal laterality or duplication, persistent patency or premature closure, and aneurysm formation

A congenital cardiovascular finding in which the arterial duct (ductus arteriosus) is open beyond the normal age of spontaneous closure.

A patent ductus arteriosus is a vascular arterial connection between the thoracic aorta and the pulmonary artery. Most commonly, a patent arterial duct has its origin from the descending thoracic aorta, just distal and opposite the origin of the left subclavian artery. The insertion of the ductus is most commonly into the very proximal left pulmonary artery at its junction with the main pulmonary artery. Origination and insertion sites can be variable, however

A congenital cardiovascular malformation in which the blood supply to the lungs is derived completely or in part from collateral vessels that arise from the aorta or its branches.

At least part of the pulmonary blood flow in derived from systemic-topulmonary collateral arteries that are highly variable in number, and that usually arise from the descending thoracic aorta, but uncommonly may originate from the aortic arch or the subclavian. carotid, or even the coronary arteries. There may or may not be native pulmonary arteries which, if present, may be hypoplastic, and either confluent or non-confluent. Systemic pulmonary collateral arteries have been categorised into 3 types based on their site of origin and the way they connect to the pulmonary circulation: direct aortopulmonary collaterals, indirect aortopulmonary collaterals, and true bronchial arteries. This term is to intended to exclude patients with "Tetralogy of Fallot with pulmonary atresia" since they are coded using the tetralogy of Fallot codes

A congenital cardiovascular malformation of a coronary artery. This includes absence of a coronary, anomalous origin or course, dilation or stenosis, and fistulas. Congenital anomalies of the coronary venous system should also be included here.
Synonyms

Pulmonary arterial sling

\section{Congenital ductus arteriosus anomaly}

Patent ductus arteriosus; PDA; open ductus arteriosus; persistent ductus arteriosus; ductus arteriosus non-closure; patent ductus arteriosus, persisting type; persistent ductus arteriosus (Botalli); persistent ductus Botalli; patent ductus Botalli

Major systemic-to-pulmonary collateral artery; systemic-topulmonary collateral vessels; systemic-to-pulmonary collateral flow; aortopulmonary collateral arteries 
Anomalous origin of coronary artery from pulmonary arterial tree

Anomalous origin of left coronary artery from pulmonary artery

Anomalous aortic origin or course of coronary artery

Anomalous aortic origin of coronary artery with ventriculo-arterial concordance

Right coronary artery from left aortic sinus with 09.46 .26 ventriculo-arterial concordance

Left coronary artery from right aortic sinus with ventriculo-arterial concordance

Anterior descending from right coronary artery across right ventricular outflow tract

Intramural proximal coronary arterial course

09.43 .05

Single coronary supplying all of heart

Myocardial bridging of coronary arter

Congenital coronary arterial orifice stenosis

09.41 .01

09.41 .03

09.43 .12

09.44 .05
A congenital cardiovascular malformation in which a coronary artery originates from the pulmonary trunk or one of its branches.

Although the most common of these malformations involves the left coronary artery arising from the pulmonary trunk (main pulmonary artery) rather than from the aorta, occasionally the right coronary artery, the circumflex, or both coronary arteries may arise from any of the central pulmonary arteries

A congenital cardiovascular malformation in which the left coronary artery originates from the pulmonary trunk or one of its branches

09.42.00 A congenital cardiovascular malformation in which the origin and/or course of a coronary artery is abnormal. This is where coronary "anomalies" in the presence of discordant ventriculo-arterial connections should be coded

A congenital cardiovascular malformation in the setting of ventriculo-arterial concordance in which a coronary artery arises from the aorta at a location other than its expected sinus. Anomalous aortic origins of the coronary arteries include a spectrum of anatomic variations of the normal coronary artery origins. Coronary artery anomalies of aortic origin to be coded under this diagnostic field include: anomalies of take-off (bigh take-off), origin (sinus), branching, and number. An anomalous course of the coronary artery vessels is also significant, particularly those coronary arteries that arise or course between the great vessels. This term is used for patients with concordant ventriculo-arterial connections

A congenital cardiovascular malformation in the setting of ventriculo-arterial concordance in which the right coronary artery arises from, or immediately above, the left sinus of Valsalva

A congenital cardiovascular malformation in the setting of ventriculo-arterial concordance in which the left coronary artery arises from, or immediately above, the right sinus of Valsalv

A congenital cardiovascular malformation in which the left circumflex coronary artery arises normally and the anterior descending coronary artery arises from the proximal right coronary artery and courses across the right ventricular outflow tract

A congenital cardiovascular malformation in which the proximal Intramural coronary artery coronary artery courses within and parallel to the wall of the aorta before it emerges to assume its epicardial course

A congenital cardiovascular malformation in which a solitary coronary artery supplies the myocardium

A congenital cardiovascular malformation in which a usually epicardial coronary arterial segment is located within the ventricular myocardium, making this segment susceptible to compression during systole right ventricular outflow tract

\section{Myocardial bridge}

A congenital cardiovascular malformation in which the orifice

ALCAPA - [Anomalous origin of left coronary artery from pulmonary artery]; ALCAPT - [Anomalous origin of left coronary artery from pulmonary trunk]

Anomalous aortic origin of coronary artery

Right coronary artery from left sinus

Left coronary artery from right sinus

Anterior interventricular artery from right coronary artery across of a coronary artery is narrowed 
ICD-11 congenital cardiology terms with

International Paediatric and Congenital

Cardiac Code (IPCCC) numbers, definitions

and synonyms

Congenital coronary arterial orifice atresia

09.44 .19

A congenital cardiovascular malformation in which the orifice of a coronary artery is not patent.

This excludes single coronary artery anomalies

Congenital coronary arterial fistula

A congenital cardiovascular malformation in which a coronary artery communicates, through an anomalous channel, with a cardiac chamber or with any segment of the pulmonary circulation.

This communication may be simple and direct or may be tortuous and dilated. In order of frequency the involved coronary artery is the right, the left and, rarely, both coronary arteries. Occasionally multiple fistulas are present. This is distinct from anomalous origin of the coronary artery

Congenital coronary arterial fistula to right

ventricle

A congenital cardiovascular malformation in which a coronary artery communicates, through an anomalous channel, with the right ventricle

Congenital coronary arterial fistula to left ventricle $\quad 09.45 .22$

Congenital coronary arterial aneurysm

09.46 .14

Congenital pericardial anomaly

10.01 .05

Pulmonary arteriovenous fistula

09.19 .05

congenital cardiovascular malformation in which a coronary artery communicates, through an anomalous channel, with the left ventricle

A congenital cardiovascular malformation in which there is one or more localised dilation(s) of a coronary vessel. It is usually defined as an increase in luminal diameter that exceeds 1.5 times the luminal diameter of the adjacent normal coronary arteries.

Coronary artery aneurysms are usually seen in two forms, saccular (shaped like a sack, with a change in calibre over a very short distance), and fusiform (shaped like a spindle, tapering towards each end), and both may be single or multiple

A congenital cardiovascular malformation in which there is a structural and/or functional abnormality of the pericardium. This would include antenatal pericardial effusion and congenital tumours of the serous pericardium

A congenital cardiovascular malformation in which there is an abnormal, direct connection between a pulmonary artery and pulmonary vein or left atrium without an intervening capillary bed.

NB This is classifed in ICD-11 under Structural developmental

Pulmonary arteriovenous malformation; pulmonary arteriovenous aneurysm 